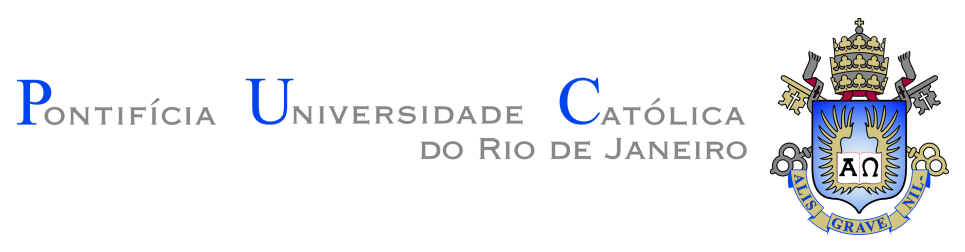

Ana Beatriz Loureiro Brito Fernandes

\title{
On MIMO Communications Systems with 1-bit Quantization and Comparator Networks at the Receiver
}

Dissertação de Mestrado

Dissertation presented to the Programa de Pós-graduação em Engenharia Elétrica of PUC-Rio in partial fulfillment of the requirements for the degree of Mestre em Engenharia Elétrica.

Advisor: Prof. Lukas Tobias Nepomuk Landau 

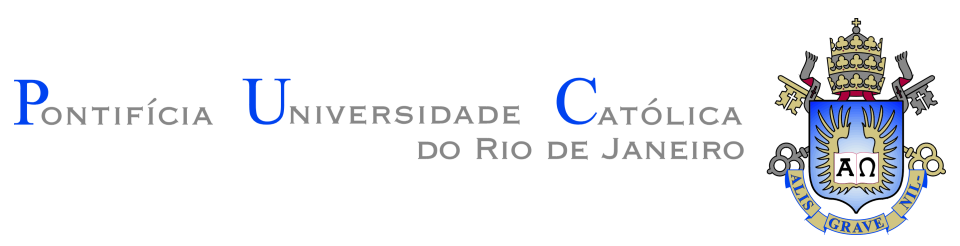

Ana Beatriz Loureiro Brito Fernandes

\section{On MIMO Communications Systems with 1-bit Quantization and Comparator Networks at the \\ Receiver}

Dissertation presented to the Programa de Pós-graduação em Engenharia Elétrica of PUC-Rio in partial fulfillment of the requirements for the degree of Mestre em Engenharia Elétrica. Approved by the Examination Committee.

Prof. Lukas Tobias Nepomuk Landau

Advisor

Centro de Estudos em Telecomunicações (CETUC) - PUC-Rio

Prof. Junil Choi

Korea Advanced Institute of Science and Technology - KAIST

Prof. Gonzalo Seco-Granados

Universitat Autònoma de Barcelona - UAB

Prof. Tadeu Nagashima Ferreira Universidade Federal Fluminense - UFF

Prof. Rodrigo Caiado de Lamare

Centro de Estudos em Telecomunicações (CETUC) - PUC-Rio 
All rights reserved.

\section{Ana Beatriz Loureiro Brito Fernandes}

She received her Bachelor of Science degree in Telecommunications Engineering, from the Universidade Federal Fluminense (UFF), in 2017.

Bibliographic data

Fernandes, Ana Beatriz Loureiro Brito

On MIMO Communications Systems with 1-bit Quantization and Comparator Networks at the Receiver / Ana Beatriz Loureiro Brito Fernandes; advisor: Lukas Tobias Nepomuk Landau. - 2021.

67 f. : il. color. ; $30 \mathrm{~cm}$

Dissertação (mestrado) - Pontifícia Universidade Católica do Rio de Janeiro, Departamento de Engenharia Elétrica, 2021.

Inclui bibliografia

1. Engenharia Elétrica - Teses. 2. Sistemas MIMO;. 3. Quantização de Baixa Resolução;. 4. MMSE;. 5. Teorema Bussgang;. 6. Rede de Comparadores;. 7. Busca Gananciosa;. 8. Estimação de Canal;. 9. Taxa Alcançável.. I. Landau, Lukas Tobias Nepomuk. II. Pontifícia Universidade Católica do Rio de Janeiro. Departamento de Engenharia Elétrica. III. Título. 


\section{Acknowledgments}

First of all, I would like to thank my advisor Prof. Lukas T. N. Landau for introducing me to this novel topic. This study would not be possible without his encouragement, patience, guidance, daily motivation and assistance.

I would like to express my love to my family, for their encouragement in both good and difficult times. To my parents, thank you for your advice, patience and support in my insane decisions throughout the years. To my mother, Márcia, thank you for teaching me the importance of learning and that the only thing people can't take from you is your knowledge. To my father, Marco, for teaching me that I should not take things so seriously: life is much simpler through his eyes. To my sister, Ana Carolina, thank you for always being there for me, your love and friendship. To my fiance, Pedro, for showing me that relaxing breaks are not only healthy but also super necessary, that I must not be anxious about things I can't control and, most importantly, that I should not settle for less than I deserve.

My sincere thanks to all professors, students and staff from CETUC for providing a pleasant environment to pursue my studies. I had the opportunity to share classes and the laboratory with some amazing people. A special thanks to Roberto and Erico for their help, great insights and friendship. I am also grateful to the members of my defense committee, professors Junil Choi, Gonzalo Seco-Granados, Tadeu N. Ferreira and Rodrigo C. de Lamare, for generously serving as referees and take their time to evaluate my thesis.

This study was financed in part by the Coordenação de Aperfeiçoamento de Pessoal de Nível Superior - Brasil (CAPES) - Finance Code 001. 


\section{Abstract}

Fernandes, Ana Beatriz Loureiro Brito; Landau, Lukas Tobias Nepomuk (Advisor). On MIMO Communications Systems with 1-bit Quantization and Comparator Networks at the Receiver. Rio de Janeiro, 2021. 67p. Dissertação de mestrado - Departamento de Engenharia Elétrica, Pontifícia Universidade Católica do Rio de Janeiro.

Multiple-input multiple-output (MIMO) systems employs an increasing number of antennas, which leads to relevant energy consumption and hardware cost of the corresponding front ends. In this context, the use of lowresolution analog to digital converters (ADCs) is promoted as a promising solution to this problem. In this study we consider a low-resolution MIMO receiver which implies that the received signals simultaneously are processed by the 1-bit ADCs and the comparator network. The input signals for the comparator network can come from different antennas, such that the comparator network extension can be interpreted as virtual channels with binary outputs. Based on such low-resolution MIMO receivers, we develop low-resolution aware linear minimum mean-squared error (LRA-LMMSE) channel estimator and detector according to the Bussgang theorem. Two comparator networks are proposed, namely, fully and partially connected networks. We also devise a greedy search-based partially connected network that can use much less comparators to approach the performance of the fully connected network. Numerical results shows that adding virtual channels can be better than adding extra physical channels which corresponds to additional receive antennas in terms of bit error rate (BER). Furthermore, by employing the proposed channel estimator and its corresponding estimation error, we build up a lower bound on the ergodic sum rate for the LRA-LMMSE receiver. Simulation results show that the systems with the proposed network-aided MIMO systems with 1-bit quantization at the receiver outperforms the conventional 1-bit MIMO system in terms of BER and mean-square error (MSE) performances. Moreover, numerical simulations confirm a significant advantage in terms of sum rate for the proposed system.

\section{Keywords}

MIMO Systems; Low-Resolution Quantization; MMSE; Bussgang theorem; Comparator Network; Greedy Search; Channel Estimation; Achievable Rate. 


\section{Resumo}

Fernandes, Ana Beatriz Loureiro Brito; Landau, Lukas Tobias Nepomuk (Orientador). Sistemas de Comunicação MIMO com Quantização de 1-bit e Redes Comparadoras no Receptor. Rio de Janeiro, 2021. 67p. Dissertação de Mestrado - Departamento de Engenharia Elétrica, Pontifícia Universidade Católica do Rio de Janeiro.

Os sistemas de múltiplas entradas e múltiplas saídas (MIMO) empregam um número crescente de antenas, o que leva a relevantes consumo de energia e custo de hardware dos front-ends correspondentes. Nesse contexto, o uso de conversores analógico-digitais (ADCs) de baixa resolução é promovido como uma solução promissora para este problema. Neste estudo consideramos um receptor MIMO de baixa resolução que implica que os sinais recebidos são processados simultaneamente pelos 1-bit ADCs e pela rede comparadora. Os sinais de entrada da rede comparadora podem vir de antenas diferentes, de modo que a extensão da rede comparadora pode ser interpretada como canais virtuais com saídas binárias. Com base nesses receptores MIMO de baixa resolução, desenvolvemos um estimador de canal e detector lineares de baixa resolução baseados no critério de mínimo erro médio quadrático (LRA-LMMSE) de acordo com o teorema de Bussgang. Duas redes de comparação são propostas, nomeadas, redes total e parcialmente conectadas. Também desenvolvemos uma rede parcialmente conectada baseada em busca gananciosa que usa muito menos comparadores para obter um desempenho bem próximo ao da rede totalmente conectada. Os resultados numéricos mostram que adicionar canais virtuais pode ser melhor do que adicionar canais físicos extras que correspondem a antenas de recepção adicionais em termos de taxa de erro de bit (BER). Além disso, ao empregar o estimador de canal proposto e seu erro de estimativa correspondente, construímos um limite inferior na taxa de soma ergódica para o receptor LRA-MMSE. Os resultados de simulação mostram que os sistemas com a proposta sistemas MIMO auxiliados por rede com quantização de 1-bit no receptor superam o convencional sistema MIMO de 1-bit em termos de desempenho de BER e erro quadrático médio (MSE). Além disso, as simulações numéricas confirmam uma vantagem significativa em termos de taxa de soma para o sistema proposto.

\section{Palavras-chave}

Sistemas MIMO; Quantização de Baixa Resolução; MMSE; Teorema Bussgang; Rede de Comparadores; Busca Gananciosa; Estimação de Canal; Taxa Alcançável. 


\section{Table of contents}

$\begin{array}{lll}1 & \text { Introduction } & 13\end{array}$

1.1 Motivation and Prior Works 13

$\begin{array}{lll}1.2 & \text { Contributions } & 14\end{array}$

$\begin{array}{ll}1.3 \text { Thesis Outline } & 15\end{array}$

$\begin{array}{ll}1.4 \text { Notation } & 16\end{array}$

$\begin{array}{ll}1.5 \text { List of Publications } & 16\end{array}$

2 Literature Review and Relevant Baselines $\quad 17$

$\begin{array}{lll}2.1 & \text { MU-MIMO Uplink System } & 17\end{array}$

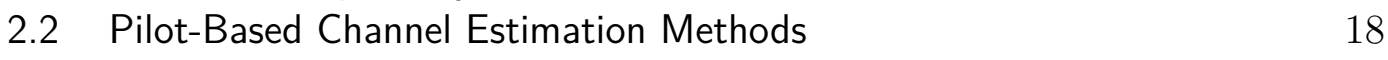

2.2.1 Standard LS Channel Estimator 18

2.2.2 Linear LRA Channel Estimators 19

$\begin{array}{ll}\text { 2.2.2.1 LRA-LS Channel Estimator } & 19\end{array}$

2.2.2.2 LRA-LMMSE Channel Estimator 20

2.2.3 nML Channel Estimator 20

2.3 MIMO Detection Methods 21

2.3.1 Linear Detectors 21

2.3.1.1 Standard MF Receive Filter $\quad 22$

2.3.1.2 Standard ZF Receive Filter $\quad 22$

2.3.1.3 Standard MMSE Receive Filter $\quad 22$

2.3.2 Linear LRA Detectors $\quad 22$

2.3.2.1 LRA-ZF Receive Filter 23

2.3.2.2 LRA-MMSE Receive Filter $\quad 23$

2.3.3 nML Detector 24

2.3.4 One-Bit Spatial Sigma-Delta Detector 26

2.4 Sum Rate Analysis 26

2.4.1 Data Transmission with LMMSE Receiver 26

2.4.2 Lower Bounding the Sum Rate 27

$\begin{array}{ll}2.5 \text { Summary } & 27\end{array}$

3 System Model 29

3.1 Proposed Comparator Network-aided MIMO Receiver 29

3.2 Comparator Network Design 31

3.2.1 Fully Connected Network 31

3.2.2 Partially Connected Network 32

$\begin{array}{ll}3.3 \text { Summary } & 32\end{array}$

$4 \quad$ Linear Channel Estimation 33

4.1 Pilot-Based Channel Estimation 33

4.2 Proposed LRA-LMMSE Channel Estimator 34

4.3 Mean-Squared Error of the Channel Estimate 35

4.4 Numerical Results $\quad 35$

$\begin{array}{lll}4.5 & \text { Summary } & 37\end{array}$ 
5 Linear Detection $\quad 38$

5.1 Proposed LRA-LMMSE Detector 38

$\begin{array}{lll}5.2 & \text { Robust LRA-LMMSE Detector } & 39\end{array}$

5.3 Greedy Search Algorithm 41

5.4 Analysis 41

5.4.1 Computational Complexity $\quad 42$

5.4.2 Computational and Hardware Costs 42

5.5 Numerical Results 42

5.5.1 Proof of Concept: More Antennas versus Comparator Network 43

5.5.2 Proposed LRA-LMMSE Detector 44

5.5.3 Robust LRA-LMMSE Detector 45

5.6 Summary 46

6 Sum Rate Analysis $\quad 48$

6.1 Data Transmission with LRA-LMMSE Receiver 48

6.2 Lower Bounding the Sum Rate 49

6.3 Numerical Results 49

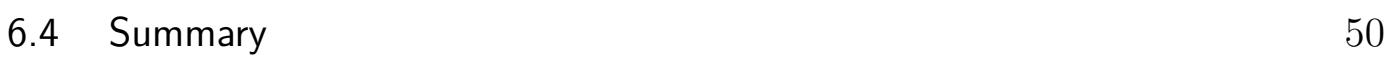

7 Conclusion and Future Work $\quad 52$

$\begin{array}{ll}\text { Bibliography } & 54\end{array}$

A Linear Channel Estimation Derivations $\quad 58$

A.1 Derivation of the Auto-Correlation Matrix of the Received Pilot Signal 58

A.2 Derivation of the LRA-LMMSE Optimal Filter and Channel Estimator 58

A.3 Derivation of the MSE of the Channel Estimate 59

B Linear Detection Derivations $\quad 61$

B.1 Derivation of the Cross-Correlation Matrix between Received and Transmitted Data Signals $\quad 61$

B.2 Derivation of the Auto-Correlation of the Received Data Signal 61

B.3 Derivation of the MSE between the Transmitted Symbol and the Filter Output for the Robust Detector 62

B.4 Derivation of the Robust Cross-Correlation Matrix between Received and Transmitted Data Signals 62

B.5 Derivation of the Robust Auto-Correlation Received Data Signal 62

C Sum Rate Derivations $\quad 64$

C.1 Derivation of the Quantization Noise Covariance Matrix 64

C.2 Derivation of the Ergodic Achievable Rate for Real-Valued User Channel 64 


\section{List of figures}

Figure 2.1 System model of MU-MIMO with 1-bit ADCs. 18

Figure 3.1 System model of MU-MIMO with 1-bit ADCs and an additional comparator network. 29

Figure 3.2 Insight of the comparator network. 30

Figure 3.3 Number of comparators required in the fully connected network.

Figure 4.1 MSE comparisons of LRA-LMMSE channel estimators in $2 \times 4$ MIMO systems with $\tau=N_{t}=2$.

Figure 4.2 MSE comparisons of LRA-LMMSE channel estimators in $2 \times 4$ MIMO systems with $\tau=2 N_{t}=4$.

Figure 5.1 BER performance of LRA-LMMSE detectors in $2 \times N_{r}$ MIMO systems.

Figure 5.2 BER performance of LRA-LMMSE detectors in $2 \times 10$ MIMO systems.

Figure 5.3 BER performance of robust LRA-LMMSE detectors in $2 \times 4$ MIMO systems.

Figure 5.4 MSE comparisons of robust LRA-LMMSE detectors in $2 \times 4$ MIMO systems.

Figure 6.1 Sum Rate comparisons of LRA-LMMSE channel estimators in $2 \times 4$ MIMO systems. 


\section{List of tables}

Table 5.1 Computational Complexity 42

Table 5.2 Computational and Hardware Costs 42 


\section{List of Abreviations}

3G - Third Generation

$4 \mathrm{G}$ - Fourth Generation

ADC - Analog-to-Digital Converter

AGC - Automatic Gain Control

AMP - Approximate Message Passing

AWGN - Additive White Gaussian Noise

BER - Bit Error Rate

BLMMSE - Bussgang Linear Minimum BPSK - Binary Phase Shift Keying

BS - Base Station

CAPES - Coordenação de Aperfeiçoamento de Pessoal de Nível Superior

CETUC - Centro de Estudos em Telecomunicações

CSI - Channel State Information

GAMP - Generalized Approximate Message Passing

IDD - Iterative Detection and Decoding

LRA - Low-Resolution Aware

LS - Least Squares

MAP - Maximum A-posteriori Probability

MF - Matched Filter

MIMO - Multiple-Input Multiple-Output

ML - Maximum Likelihood

MMSE - Minimum Mean Square Error

MRC - Maximum Ratio Combiner

MSE - Mean Square Error

MU - Multiuser

nML - near Maximum Likelihood

PSK - Phase Shift Keying

QPSK - Quadrature Phase-Shift Keying

RLS - Recursive Least Squares

$\Sigma \Delta$ - Sigma-Delta

SINR - Signal-to-Interference-plus-Noise Ratio

SNR - Signal-to-Noise Ratio

ZF - Zero-Forcing 


\section{Introduction}

In this chapter the research background and the motivations of this thesis are presented. Then, the main contributions and the structure of this study are provided. Also, the notations used throughout the thesis are introduced. The last section shows a list of the publications generated during the period of working on this thesis.

\section{1}

\section{Motivation and Prior Works}

The future of wireless communications, with networks that scale up in speed and bandwidth, has as a promising technical candidate known as large-scale (or massive) MIMO systems. Massive MIMO systems have many advantages when compared to current systems and have attracted much attention due to its large improvement in spectral efficiency and mitigation of the propagation loss caused by channel fading, among others [1]. However, there are still some practical challenges when it comes to the deployment of a large number of antennas at the base station (BS), such as hardware cost and power consumption. For instance, the power consumption of ADCs scales exponentially in the number of quantization bits [2]. Therefore, the use of current high-speed and high-resolution ADCs (8-12 bits) for each antenna array would become a great burden to the BS. Consequently, the use of low-cost and low-resolution ADCs (1-3 bits) are promoted as a solution to this problem $[3,4,5]$.

Many works have studied large-scale MIMO systems with low resolution ADCs at the front-end. Specifically, 1-bit ADCs are of interest in such systems due to their demand of very low power. A common used technique to mitigate the performance loss caused by the coarse quantization is oversampling, where the received signal is sampled at a faster than Nyquist rate [6]. In this regard, the studies in $[7,8,9,10]$ have considered temporal oversampling at the receiver in order to achieve better estimation and detection performances. However, for temporal oversampling, the computational complexity is relatively high for the receive processing.

The channel estimation is a problem that currently limits the perfor- 
mance of coarsely quantized systems. Several papers have investigated channel estimation for quantized massive MIMO systems such as least squares (LS) [11], recursive least squares (RLS) [12], approximate message passing (AMP) [13] and generalized approximate message passing (GAMP) [14]. Another sophisticated channel estimator is given by the near maximum likelihood (nML) estimator devised in [15]. The authors in [16] developed a Bussgang linear minimum mean squared error (BLMMSE) channel estimator, where it has also been derived lower bounds on the theoretical achievable rate for maximum ratio combiner (MRC) and zero-forcing (ZF) receivers. Moreover, maximum a-posteriori probability (MAP) channel estimators and their performance analysis have been studied in $[17,18]$.

The work in [19] develops a channel estimator by taking spatial and temporal correlations into consideration. Furthermore, the low complexity channel estimator and its corresponding achievable rate from [20] relies on a model with infinite number of channel taps, and independent and identically distributed quantization noise.

For signal detection with low-resolution ADCs, different strategies exist in literature, for example: iterative detection and decoding (IDD) [21], sphere decoding [22] and nML [15]. Moreover, coarse quantization in large-scale MIMO systems has been considered in a number of different aspects and approaches. The design of a proper automatic gain control (AGC) can be relevant for low resolution ADCs (more than 1-bit) in order to minimize the signal distortion due to the coarse quantization [23]. Recently, the authors in $[24,25]$ have used spatial oversampling by employing a 1-bit Sigma-Delta $(\Sigma \Delta)$ sampling scheme which have shown large performance gains on channel estimation and signal detection. Furthermore, the studies in [26, 27, 28, 29] have devised different precoding techniques which rely on 1-bit quantization at the transmitter.

\section{2}

\section{Contributions}

The scope of this thesis lies in the design and understanding of a novel MIMO receiver architecture that uses coarse quantization ADCs. The proposed MIMO receiver includes a comparator network with binary outputs which can compare signals from different antennas in terms of real and imaginary parts. The addition of the comparator network can be interpreted as a number of additional virtual channels which can contain additional information that aids the detection and channel estimation processes. In this context, channel estimation and signal detection algorithms are developed for the proposed 
system.

Two types of comparator networks are proposed, namely, fully and partially connected networks. In this context, for the design of the partially connected network a Greedy Search-based algorithm is devised. With the optimized partially connected network, much less comparators are required to approach a comparable performance of the fully connected network. Finally, numerical results confirm that the BER and MSE for detection and channel estimation can be significantly reduced when adding a comparator network. Based on the proposed detector, a sum rate analysis is presented. Simulation results confirm that the proposed system yields significantly sum rate performance in comparison to the conventional 1-bit MIMO receiver.

The contributions can be summarized as follows:

- Introduction of a novel MIMO receiver architecture with coarse quantization using partially or fully connected comparator networks.

- Development of LRA-LMMSE channel estimation for the novel system, published in [30].

- Development of LRA-LMMSE detector for the novel system, published in [31] and a robust version of it that takes into account the channel estimation error statistics.

- Sum rate analysis in terms of construction of a lower bound, published in [30].

\section{3 \\ Thesis Outline}

This thesis is organized as follows:

- Chapter 2 gives some technical background on this thesis;

- Chapter 3 shows the overall proposed system model and describes the insight and design of the comparator network;

- Chapter 4 derives the pilot-based LRA-LMMSE channel estimator for the proposed system;

- Chapter 5 derives the LRA-LMMSE detector and a robust version of it. For designing the partially connected network, a greedy search algorithm is also devised. Moreover, computational and hardware costs are provided;

- Chapter 6 presents the construction of the lower bound on the sum rate;

- Chapter 7 displays the conclusions and discusses possible extensions for the presented study; 


\section{4 \\ Notation}

Regarding the notation, bold upper and lower case letters such as $\mathbf{A}$ and a denote matrices and vectors, respectively. $\mathbf{I}_{n}$ is a $n \times n$ identity matrix. The vector or matrix transpose and conjugate transpose are represented by $(\cdot)^{T}$ and $(\cdot)^{H} \cdot \mathfrak{R}\{\cdot\}$ and $\mathfrak{I}\{\cdot\}$ get the real and imaginary part from the corresponding vector or matrix, respectively. Additionally, $\operatorname{diag}(\mathbf{A})$ is a diagonal matrix only containing the diagonal elements of $\mathbf{A}$. The inverse of sine function is denoted by $\sin ^{-1}(\cdot)$. Moreover, $\operatorname{vec}(\mathbf{A})$ is the vectorized form of $\mathbf{A}$ obtained by stacking its columns, while the inverse of this operation is unvec(a), depending on the context. The expectation operator is denoted as $E[\cdot]$. Finally, $\otimes$ is the Kronecker product.

\section{5}

\section{List of Publications}

The work presented in this thesis gave rise to the following papers:

1. Comparator Network Aided Detection for MIMO Receivers with 1-bit Quantization in 2020 54th Asilomar Conference on Signals, Systems and Computers, 2020, pp. 384-387, doi: 10.1109/IEEECONF51394.2020.9443453.

2. Comparator Network Aided Channel Estimation and Achievable Rates for MIMO Receivers with 1-bit Quantization in IEEE Statistical Signal Processing (SSP) Workshop 2021, Rio de Janeiro, Brazil, 2021. 


\section{2 \\ Literature Review and Relevant Baselines}

In this chapter, we review some concepts related to this thesis, which are important for understanding some of the techniques used in the remaining chapters. For advanced readers, this chapter can be skipped. The following sections briefly introduce some topics related to the MIMO systems including pilot-based channel estimation, MIMO detection and sum rate analysis methods.

\section{1}

\section{MU-MIMO Uplink System}

The multiuser multiple-input multiple-output (MU-MIMO) system allows the transmission and reception of more than one data signal simultaneously, achieving very high data rates over wireless links. This technique has become a key component of different wireless communication standards such as WIFI, third generation $(3 \mathrm{G})$ and fourth generation $(4 \mathrm{G})$. The general system model is illustrated in Fig. 2.1. An uplink single-cell MIMO system with $N_{t}$ single-antenna users and $N_{r}$ receive antennas is considered, with $N_{r}>N_{t}$, written as

$$
\mathbf{y}=\sum_{k=1}^{N_{t}} \mathbf{h}_{k} x_{k}+\mathbf{n}=\mathbf{H} \mathbf{x}+\mathbf{n},
$$

where the vector $\mathbf{x}$ contains complex transmit symbols of the $N_{t}$ users which have unit power normalization, $\mathbf{H} \in \mathbb{C}^{N_{r} \times N_{t}}$ is the channel matrix and $\mathbf{n} \in \mathbb{C}^{N_{r} \times 1}$ is the noise vector where each element has a variance $\sigma_{n}^{2}$.

The received signal is then forwarded to the 1-bit ADCs. Letting $\mathcal{Q}(\cdot)$ represent the 1-bit quantization, the input of the detector is described by

$$
\mathbf{y}_{\mathcal{Q}}=\mathcal{Q}(\mathbf{y})=\mathcal{Q}(\mathfrak{R}\{\mathbf{y}\})+j \mathcal{Q}(\mathfrak{I}\{\mathbf{y}\}),
$$

where $\mathfrak{R}\{\cdot\}$ and $\mathfrak{I}\{\cdot\}$ denote the real and imaginary parts, respectively. Then, the real and imaginary parts are element-wisely quantized as $\left\{ \pm \frac{1}{\sqrt{2}}\right\}$ based on the sign. 


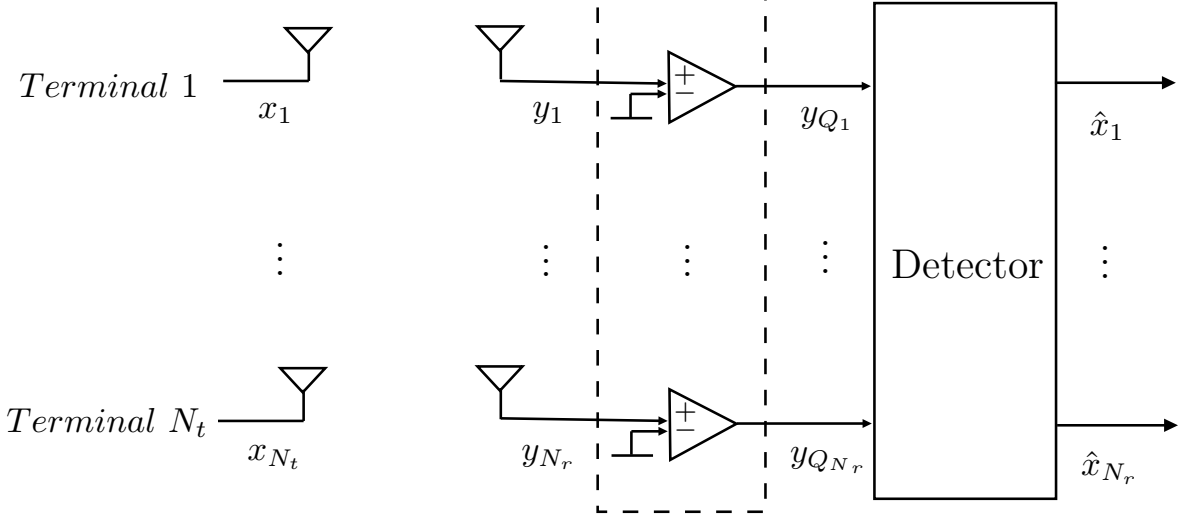

Figure 2.1: System model of MU-MIMO with 1-bit ADCs.

\section{2}

\section{Pilot-Based Channel Estimation Methods}

In a practical system, the channel state information (CSI) is estimated at the BS and this knowledge is used to detect the data symbols transmitted by the users. Each transmission block is divided into two sub-blocks: one dedicated to the pilot symbols and the other to the data symbols. The part containing the pilots can be either located at the beginning of each block or spread according to a desired pattern [32]. During the training phase, each terminal simultaneously transmits sequences of $\tau$ pilot symbols to the BS, which yields

$$
\mathbf{Y}_{p}=\mathbf{H} \Phi^{T}+\mathbf{N}_{p}
$$

where $\mathbf{Y}_{p} \in \mathbb{C}^{N_{r} \times \tau}$ is the matrix containing the unquantized received signal, $\Phi \in \mathbb{C}^{\tau \times N_{t}}$ is the matrix of pilot symbols and $\mathbf{N}_{p}$ is the noise matrix. Vectorizing the received signal yields

$$
\operatorname{vec}\left(\mathbf{Y}_{p}\right)=\mathbf{y}_{p}=\tilde{\mathbf{\Phi}} \mathbf{h}+\mathbf{n}_{p}
$$

where $\tilde{\boldsymbol{\Phi}}=\left(\boldsymbol{\Phi} \otimes \mathbf{I}_{N_{r}}\right), \mathbf{h}=\operatorname{vec}(\mathbf{H})$ and $\mathbf{n}_{p}=\operatorname{vec}\left(\mathbf{N}_{p}\right)$. Then, the quantized signal can be expressed as $\mathbf{y}_{\mathcal{Q}_{p}}=\mathcal{Q}\left(\mathbf{y}_{p}\right)$.

\subsection{1}

\section{Standard LS Channel Estimator}

The authors in [11] have proposed the standard LS estimator for 1-bit non-oversampled systems, which can be computed according to

$$
\begin{aligned}
\hat{\mathbf{h}}_{\mathrm{LS}} & =\arg \min _{\overline{\mathbf{h}}} E\left[\left\|\mathbf{y}_{\mathcal{Q}_{p}}-\tilde{\boldsymbol{\Phi}} \overline{\mathbf{h}}\right\|_{2}^{2}\right] \\
& =\left(\tilde{\boldsymbol{\Phi}}^{H} \tilde{\boldsymbol{\Phi}}\right)^{-1} \tilde{\boldsymbol{\Phi}}^{H} \mathbf{y}_{\mathcal{Q}_{p}} .
\end{aligned}
$$


The main advantage of this channel estimator is that no prior information is needed at the receiver.

\subsection{2}

\section{Linear LRA Channel Estimators}

According to the works in $[9,16]$, the quantized received signal can be rewritten as

$$
\mathbf{y}_{\mathcal{Q}_{p}}=\hat{\mathbf{\Phi}} \mathbf{h}+\tilde{\mathbf{n}}_{p}
$$

where the right hand side corresponds to a linear model that relies on the Bussgang decomposition approach. In this context, the linear model involves the matrix $\hat{\boldsymbol{\Phi}}=\mathbf{A}_{p} \tilde{\boldsymbol{\Phi}}$ and the effective noise vector $\tilde{\mathbf{n}}_{p}=\mathbf{A}_{p} \mathbf{n}_{p}+\mathbf{n}_{q, p}$, with $\mathbf{A}_{p}$ being a well chosen square matrix and $\mathbf{n}_{q, p}$ being the quantization noise. The Bussgang-based linear operator $\mathbf{A}_{p}$ is chosen to make the quantization noise uncorrelated with $\mathbf{y}_{p}$ [33], and is given by

$$
\mathbf{A}_{p}=\mathbf{C}_{\mathbf{y}_{p} \mathbf{y}_{\mathcal{Q}_{p}}}^{H} \mathbf{C}_{\mathbf{y}_{p}}^{-1}=\sqrt{\frac{2}{\pi}} \mathbf{K}_{p},
$$

where $\mathbf{K}_{p}=\operatorname{diag}\left(\mathbf{C}_{\mathbf{y}_{p}}\right)^{-\frac{1}{2}}$ and the cross-correlation matrix between the received signal $\mathbf{y}_{p}$ and its quantized signal $\mathbf{y}_{\mathcal{Q}_{p}}$ is given by

$$
\mathbf{C}_{\mathbf{y}_{p} \mathbf{y}_{\mathcal{Q}_{p}}}=\sqrt{\frac{2}{\pi}} \mathbf{K}_{p} \mathbf{C}_{\mathbf{y}_{p}}=\mathbf{A}_{p} \mathbf{C}_{\mathbf{y}_{p}},
$$

where the auto-correlation matrix of $\mathbf{y}_{p}$ yields

$$
\mathbf{C}_{\mathbf{y}_{p}}=\tilde{\boldsymbol{\Phi}} \mathbf{R}_{\mathbf{h}} \tilde{\boldsymbol{\Phi}}^{H}+\mathbf{C}_{\mathbf{n}_{\mathbf{p}}}
$$

with $\mathbf{R}_{\mathbf{h}}=E\left[\mathbf{h h}^{H}\right]$ and $\mathbf{C}_{\mathbf{n}_{\mathbf{p}}}=E\left[\mathbf{n}_{p} \mathbf{n}_{p}^{H}\right]$.

Then, based on the statistically equivalent linear model in (2-6), two basic approaches for the channel estimation are presented in the next subsections.

\subsubsection{1}

\section{LRA-LS Channel Estimator}

The LRA-LS channel estimator based on the Bussgang decomposition is obtained by solving the following optimization problem

$$
\begin{aligned}
\hat{\mathbf{h}}_{\mathrm{LRA}-\mathrm{LS}} & =\arg \min _{\overline{\mathbf{h}}} E\left[\left\|\mathbf{y}_{\mathcal{Q}_{p}}-\hat{\mathbf{\Phi}} \overline{\mathbf{h}}\right\|_{2}^{2}\right] \\
& =\left(\hat{\boldsymbol{\Phi}}^{H} \hat{\boldsymbol{\Phi}}\right)^{-1} \hat{\boldsymbol{\Phi}}^{H} \mathbf{y}_{\mathcal{Q}_{p}} .
\end{aligned}
$$

Compared to the standard LS channel estimator, this estimator implicitly takes $\mathbf{R}_{\mathbf{h}}$ into account as $\mathbf{R}_{\mathbf{h}}$ is used for computing the linear operator $\mathbf{A}_{p}$. 


\subsubsection{2}

\section{LRA-LMMSE Channel Estimator}

The LRA-LMMSE optimal filter can be obtained through the optimization problem formulated as

$$
\begin{aligned}
\mathbf{W}_{\text {LRA-LMMSE }} & =\arg \min _{\mathbf{W}} E\left[\left\|\mathbf{h}-\mathbf{W}_{\mathbf{Y}_{\mathcal{Q}}}\right\|_{2}^{2}\right] \\
& =\mathbf{R}_{\mathbf{h}} \hat{\boldsymbol{\Phi}}^{H} \mathbf{C}_{\mathbf{y}_{\mathcal{Q} p}}^{-1},
\end{aligned}
$$

where the auto-correlation of the quantized signal is calculated as [34]

$$
\mathbf{C}_{\mathbf{y}_{\mathcal{Q}_{p}}}=\frac{2}{\pi}\left(\sin ^{-1}\left(\mathbf{K}_{p} \Re\left\{\mathbf{C}_{\mathbf{y}_{p}}\right\} \mathbf{K}_{p}\right)+j \sin ^{-1}\left(\mathbf{K}_{p} \mathfrak{I}\left\{\mathbf{C}_{\mathbf{y}_{p}}\right\} \mathbf{K}_{p}\right)\right)
$$

which is known as the arcsine law. Then, the resulting LRA-LMMSE channel estimator corresponds to the linear operation

$$
\hat{\mathbf{h}}_{\text {LRA-LMMSE }}=\mathbf{R}_{\mathbf{h}} \hat{\boldsymbol{\Phi}}^{H} \mathbf{C}_{\mathbf{y}_{\mathcal{Q}_{p}}}^{-1} \mathbf{y}_{\mathcal{Q}_{p}} .
$$

The LMMSE channel estimator has superior performance in terms of MSE when compared to the LS channel estimator. However, it suffers from a higher computational complexity.

\subsection{3}

\section{nML Channel Estimator}

The near Maximum Likelihood method in [15] makes use of a modified general system model. The complex received signal $\mathbf{y}$, before going into the quantizer, is changed by the transformation from a complex into a real-valued system. Moreover, the roles of the channel and the pilot signal are reversed. Thus and by using the scaling factor $\rho$, equation (2-3) for the $n$th receive antenna can be rewritten as

$$
\underbrace{\left[\begin{array}{c}
\operatorname{Re}\left(\mathbf{y}_{p, n}\right) \\
\operatorname{Im}\left(\mathbf{y}_{p, n}\right)
\end{array}\right]}_{\mathbf{y}_{\mathbf{R}_{p, n}}}=\sqrt{\rho} \underbrace{\left[\begin{array}{cc}
\operatorname{Re}(\boldsymbol{\Phi}) & -\operatorname{Im}(\boldsymbol{\Phi}) \\
\operatorname{Im}(\boldsymbol{\Phi}) & \operatorname{Re}(\boldsymbol{\Phi})
\end{array}\right]}_{\mathbf{\Phi}_{\mathrm{R}}} \underbrace{\left[\begin{array}{c}
\operatorname{Re}\left(\mathbf{h}_{n}\right) \\
\operatorname{Im}\left(\mathbf{h}_{n}\right)
\end{array}\right]}_{\mathbf{h}_{\mathbf{R}_{n}}}+\underbrace{\left[\begin{array}{c}
\operatorname{Re}\left(\mathbf{n}_{p, n}\right) \\
\operatorname{Im}\left(\mathbf{n}_{p, n}\right)
\end{array}\right]}_{\mathbf{n}_{\mathbf{R}, n}} .
$$

Then, the output of the quantizer is denoted by $\mathbf{y}_{\mathcal{Q}_{p, n}}=\mathcal{Q}\left(\mathbf{y}_{\mathrm{R}_{p, n}}\right)$. For the approach in [15], the real and imaginary parts of the unquantized received signal $\mathbf{y}_{\mathrm{R}_{p, n}}$ are element-wisely quantized to $\{ \pm 1\}$. With this, the first $\tau$ received signals during the training phase can be rewritten as

$$
\mathbf{y}_{\mathrm{R}_{p, n}}=\sqrt{\rho} \boldsymbol{\Phi}_{\mathrm{R}}^{T} \mathbf{h}_{\mathrm{R}_{n}}+\mathbf{n}_{\mathrm{R}_{p, n}} .
$$

Note that for simplification we skipped the fading block index used in [15].

In this context, the ML channel estimator is given by 


$$
\check{\mathbf{h}}_{\mathrm{R}_{n, M L}}=\arg \max _{\mathbf{h}_{\mathrm{R}} \in \mathbb{R}^{2 N_{t} \times 1}} \sum_{i=1}^{2 \tau} \log \left(\Psi\left(\sqrt{2 \rho} \overline{\mathbf{\Phi}}_{\mathrm{R}_{i}}^{T} \dot{\mathbf{h}}_{\mathrm{R}}\right)\right),
$$

where $\Psi(t)=\int_{-\infty}^{t} \frac{1}{\sqrt{2 \pi}} e^{-\frac{\tau^{2}}{2}} d \tau$ and $\overline{\mathbf{\Phi}}_{\mathrm{R}_{i}}$ denotes the multiplication of the correspondent $i$ th row of the pilot matrix $\boldsymbol{\Phi}_{\mathrm{R}_{i}}$ and the quantized received signal $\mathbf{y}_{\mathcal{Q}_{p, n, i}}$ at the $n$th receive antenna. Because $\Psi(\cdot)$ is a log-concave function, equation (2-16) can be solved by using standard convex optimization methods. By using prior knowledge about the norm of the channel, for example $E\left[\left\|\mathbf{h}_{\mathrm{R}_{n}}\right\|^{2}\right]=N_{t}$, which is a common assumption for channel estimation, the channel estimator in (2-16) can be improved by taking into account the norm constraint on $\hat{\mathbf{h}}_{\mathrm{R}}$. Then, the corresponding optimization problem reads as

$$
\check{\mathbf{h}}_{\mathrm{R}_{n, M L}}=\arg \max _{\substack{\mathbf{h}_{R} \in \mathbb{R}^{2 N_{t} \times 1} \\\left\|\hat{\mathbf{h}}_{\mathrm{R}}\right\|^{2} \leq N_{t}}} \sum_{i=1}^{2 \tau} \log \left(\Psi\left(\sqrt{2 \rho} \overline{\mathbf{\Phi}}_{\mathrm{R}_{i}}^{T} \dot{\mathbf{h}}_{\mathrm{R}}\right)\right) .
$$

\section{3}

\section{MIMO Detection Methods}

For the detection process, there are several known state-of-art approaches. Some of these methods are going to be presented further.

\subsection{1}

\section{Linear Detectors}

The linear detectors can be considered as filtering, because the detection process can reduce the interference. The consideration of linear detection does not yields an optimal detection performance but corresponds to a low computational complexity.

With a linear MIMO receiver, one can compute an estimate on the transmit symbol by

$$
\tilde{\mathbf{x}}=\mathbf{G y}
$$

where $\mathbf{G}$ is the filtering (or linear receive filter) matrix with size $N_{t} \times$ $N_{r}$. The filter matrix $\mathbf{G}$ can be designed according to different criteria. Next we are going to see some of this criteria. The filtering process is followed by a mapping process which maps the symbol to a symbol in the input alphabet. Typically, the symbols are mapped according to the smallest Euclidean distance. According to prior literature, the most popular linear detectors are matched filter (MF), ZF and MMSE [35]. 


\subsubsection{1}

\section{Standard MF Receive Filter}

Let us begin with the MF-type detector for unquantized receive signals (full resolution in amplitude and phase), which has the lowest computational complexity among all MIMO detectors. The MF method has the objective of maximizing the signal-to-noise ratio (SNR) at the receiver and its linear receive filter matrix is given by

$$
\mathbf{G}_{\mathrm{MF}}=a \mathbf{H}^{H},
$$

where $a$ is a scalar which can be chosen arbitrarily.

\subsubsection{2}

\section{Standard ZF Receive Filter}

The ZF approach for unquantized receive signals implies that the equalizer is unbiased. The ZF optimal filter can be obtained by solving the following optimization problem

$$
\mathbf{G}_{\mathrm{ZF}}=\arg \min _{\mathbf{G}} E\left[\|\mathbf{x}-\mathbf{G} \mathbf{y}\|_{2}^{2}\right] \text { s.t. } \mathbf{G H}=\mathbf{I} .
$$

Then, the ZF-type receive filter can be written as

$$
\mathbf{G}_{\mathrm{ZF}}=\left(\mathbf{H}^{H} \mathbf{H}\right)^{-1} \mathbf{H}^{H} .
$$

\subsubsection{3}

\section{Standard MMSE Receive Filter}

In general, the MMSE criterion relies on minimizing the MSE between the actual transmitted data and the equalizer's output with the linear receive filter matrix. In a nutshell, the MMSE filter for unquantized receive signals corresponds to the following optimization problem and solution

$$
\begin{aligned}
\mathbf{G}_{\mathrm{MMSE}} & =\arg \min _{\mathbf{G}} E\left[\|\mathbf{x}-\mathbf{G} \mathbf{y}\|_{2}^{2}\right] \\
& =\left(\frac{N_{t} \sigma_{n}^{2}}{E_{t x}} \mathbf{I}_{N_{t}}+\mathbf{H}^{H} \mathbf{H}\right)^{-1} \mathbf{H}^{H},
\end{aligned}
$$

where a point by point system is considered with $E\left[\mathbf{x x}^{H}\right]=\frac{E_{t x}}{N_{t}} \mathbf{I}_{N_{t}}$ and $E_{t x}$ being the transmit energy or power.

\subsection{2}

\section{Linear LRA Detectors}

Different to the prior mentioned detectors, the methods in this subsection imply quantized data where low resolution ADCs are considered. The use of coarse quantization is beneficial in terms of cost and power consumption but 
implicates in performance degradation in comparison to MIMO detection with full resolution. The idea of LRA detectors is to take into account the 1-bit quantization in the receive processing. In order to compute the LRA receive filters, it is necessary to rewrite the quantized signal with a linear model. As we can see in [8], based on the Bussgang decomposition the quantized received signal $\mathbf{y}_{\mathcal{Q}}$ can be rewritten as

$$
\mathbf{y}_{\mathcal{Q}}=\mathbf{A y}+\mathbf{n}_{q}
$$

where the matrix $\mathbf{A}$ is the linear operator which is chosen to make $\mathbf{n}_{q}$ uncorrelated with $\mathbf{y}[36,37]$. Then, the vector $\mathbf{n}_{q}$ is termed the statistically equivalent quantization noise. The linear operator is given by the equation

$$
\mathbf{A}=\mathbf{C}_{\mathbf{y y}_{\mathcal{Q}}}^{H} \mathbf{C}_{\mathbf{y}}^{-1}=\sqrt{\frac{2}{\pi}} \mathbf{K}
$$

with $\mathbf{K}=\operatorname{diag}\left(\mathbf{C}_{\mathbf{y}}\right)^{-\frac{1}{2}}$. The cross-correlation matrix between the received signal $\mathbf{y}$ and its quantized signal $\mathbf{y}_{\mathcal{Q}}$ is denoted by

$$
\mathbf{C}_{\mathbf{y y}_{\mathcal{Q}}}=\sqrt{\frac{2}{\pi}} \mathbf{K C}_{\mathbf{y}}
$$

where the auto-correlation of $\mathbf{y}$ is given by

$$
\mathbf{C}_{\mathbf{y}}=\mathbf{H C}_{\mathbf{x}} \mathbf{H}^{H}+\mathbf{C}_{\mathbf{n}} \text {, }
$$

with $\mathbf{C}_{\mathbf{x}}=E\left[\mathbf{x x}^{H}\right]=\mathbf{I}_{N_{t}}$ and $\mathbf{C}_{\mathbf{n}}=\mathrm{E}\left[\mathbf{n n}^{H}\right]=\sigma_{n}^{2} \mathbf{I}_{N_{r}}$.

\subsubsection{1}

\section{LRA-ZF Receive Filter}

The LRA-ZF filter minimizes the MSE as

$$
\mathbf{G}_{\mathrm{LRA}-\mathrm{ZF}}=\arg \min _{\mathbf{G}} \mathrm{E}\left[\left\|\mathbf{G}\left(\mathbf{A n}+\mathbf{n}_{q}\right)\right\|_{2}^{2}\right] \text { s.t. } \mathbf{G A H}=\mathbf{I} .
$$

By equating the gradient of the Lagrangian multiplier to zero, we obtain

$$
\mathbf{G}_{\text {LRA-ZF }}=\left(\mathbf{H}^{H} \mathbf{A}^{H} \mathbf{C}_{\mathbf{n}}^{-1} \mathbf{A} \mathbf{H}\right)^{-1} \mathbf{H}^{H} \mathbf{A}^{H} \mathbf{C}_{\mathbf{n}}^{-1},
$$

where $\mathbf{C}_{\mathbf{n}}=\mathbf{A} \mathbf{C}_{\mathbf{n}} \mathbf{A}^{H}+\mathbf{C}_{\mathbf{n}_{q}}$ and $\mathbf{C}_{\mathbf{n}_{q}}=\mathbf{C}_{\mathbf{y}_{\mathcal{Q}}}-\mathbf{A} \mathbf{C}_{\mathbf{y}} \mathbf{A}^{H}$ are the covariance matrices of the noise and the quantization noise, respectively.

\subsubsection{2}

\section{LRA-MMSE Receive Filter}

The LRA-MMSE optimization problem and solution for finding the optimal linear receiver is formulated as 


$$
\begin{aligned}
\mathbf{G}_{\text {LRA-MMSE }} & =\arg \min _{\mathbf{G}} E\left[\left\|\mathbf{x}-\mathbf{G y}_{\mathcal{Q}}\right\|_{2}^{2}\right] \\
& =\mathbf{C}_{\mathbf{y}_{\mathcal{Q}}}^{-1} \mathbf{C}_{\mathbf{y}_{\mathcal{Q}} \mathbf{x}}
\end{aligned}
$$

where the involved covariance matrix $\mathbf{C}_{\mathbf{y}_{\mathcal{Q}}}$ is calculated as [34]

$$
\mathbf{C}_{\mathbf{y}_{\mathcal{Q}}}=\frac{2}{\pi}\left(\sin ^{-1}\left(\mathbf{K} \Re\left\{\mathbf{C}_{\mathbf{y}}\right\} \mathbf{K}\right)+j \sin ^{-1}\left(\mathbf{K} \mathfrak{I}\left\{\mathbf{C}_{\mathbf{y}}\right\} \mathbf{K}\right)\right)
$$

and the cross-correlation matrix $\mathbf{C}_{\mathbf{y}_{\mathcal{Q}}}$ is based on the Bussgang theorem [36]

$$
\mathbf{C}_{\mathbf{y}_{\mathcal{Q}} \mathbf{x}}=\sqrt{\frac{2}{\pi}} \mathbf{K C}_{\mathbf{y x}}=\sqrt{\frac{2}{\pi}} \mathbf{K H C}_{\mathbf{x}}
$$

\subsection{3}

\section{nML Detector}

The nML detector is an approach for coarse quantization in terms of 1-bit quantization. The complexity of the conventional ML detector grows exponentially with the number of users due to the exhaustive search over all possible transmitted vectors. Therefore, the strategies presented at this subsection try to overcome this issue by proposing a nML detector which can provide improvement of the performance-complexity trade-off in comparison with the conventional ML detector. The main goal is to convert the ML estimation problem into a convex problem. Thus, the reformulation in [15] can be done by relaxing constraints on the transmitted vector.

First of all, the complex received signal $\mathbf{y}$, before going into the quantizer, is changed by the transformation from a complex into a real-valued system. Thus, equation (2-1) for the $n$th receive antenna can be rewritten as

$$
\underbrace{\left[\begin{array}{c}
\operatorname{Re}\left(\mathbf{y}_{n}\right) \\
\operatorname{Im}\left(\mathbf{y}_{n}\right)
\end{array}\right]}_{\mathbf{y}_{\mathrm{R}_{n}}}=\sqrt{P} \underbrace{\left[\begin{array}{cc}
\operatorname{Re}\left(\mathbf{H}_{n}\right) & -\operatorname{Im}\left(\mathbf{H}_{n}\right) \\
\operatorname{Im}\left(\mathbf{H}_{n}\right) & \operatorname{Re}\left(\mathbf{H}_{n}\right)
\end{array}\right]}_{\mathbf{H}_{\mathrm{R}_{n}}} \underbrace{\left[\begin{array}{c}
\operatorname{Re}(\mathbf{x}) \\
\operatorname{Im}(\mathbf{x})
\end{array}\right]}_{\mathbf{x}_{\mathrm{R}}}+\underbrace{\left[\begin{array}{c}
\operatorname{Re}\left(\mathbf{n}_{n}\right) \\
\operatorname{Im}\left(\mathbf{n}_{n}\right)
\end{array}\right]}_{\mathbf{n}_{\mathrm{R}_{n}}},
$$

where $P$ is the power of the transmit data and the SNR is given by the equation $\rho=\frac{P}{\sigma_{n}^{2}}$. Then, the output of the quantizer is denoted by $\mathbf{y}_{\mathcal{Q}_{n}}=\mathcal{Q}\left(\mathbf{y}_{\mathrm{R}_{n}}\right)$. For the approach in [15], the real and imaginary parts of the unquantized received signal $\mathbf{y}_{\mathbf{R}_{n}}$ are element-wisely quantized to $\{ \pm 1\}$. In this context, we have

$$
\mathbf{y}_{\mathrm{R}_{n}}=\sqrt{P} \mathbf{H}_{\mathrm{R}_{n}} \mathbf{x}_{\mathrm{R}}+\mathbf{n}_{\mathrm{R}_{n}} .
$$

With this, the reformulated ML detector problem can be defined as

$$
\hat{\mathbf{x}}_{\mathrm{R}, \mathrm{ML}}=\arg \max _{\dot{\mathbf{x}}_{\mathrm{R}} \in \mathcal{S}_{\mathrm{R}}^{N_{t}}} \prod_{i=1}^{2} \prod_{n=1}^{N_{r}} \Psi\left(\sqrt{2 \rho} \bar{h}_{\mathrm{R}_{n, i}}^{T} \dot{\mathbf{x}}_{\mathrm{R}}\right),
$$


where $\Psi(t)=\int_{-\infty}^{t} \frac{1}{\sqrt{2 \pi}} e^{-\frac{\tau^{2}}{2}} d \tau$ and $\mathcal{S}_{\mathrm{R}}^{N_{t}}$ is the $N_{t^{-}}$ary Cartesian product set of $\mathcal{S}_{\mathrm{R}}$ which is the $M$-ary data symbol constellation, ordered with the real parts of the constellations first and the imaginary parts later. Moreover, the term $\bar{h}_{\mathrm{R}_{n, i}}$ denote the multiplication of the correspondent $i$ th row of the channel $h_{\mathrm{R}_{n, i}}$ and the quantized received signal $y_{\mathcal{Q}_{n, i}}$ at the $n$th receive antenna. By relaxing the constraint $\dot{\mathbf{x}}_{\mathrm{R}} \in \mathcal{S}_{\mathrm{R}}^{N_{t}}$ in equation (2-34) we get

$$
\check{\mathbf{x}}_{\mathrm{R}, \mathrm{ML}}^{(1)}=\arg \max _{\substack{\dot{\mathbf{x}}_{\mathrm{R}} \in \mathbb{R}^{2 N_{t} \times 1} \\\left\|\dot{\mathbf{x}}_{\mathrm{R}}\right\|^{2}=N_{t}}} \sum_{i=1}^{2} \sum_{n=1}^{N_{r}} \Psi\left(\sqrt{2 \rho} \bar{h}_{\mathrm{R}_{n, i}}^{T} \dot{\mathbf{x}}_{\mathrm{R}}\right) .
$$

Although the function $\Psi(\cdot)$ is log-concave, in general, the optimization problem described above is not convex due to the norm constraint $\left\|\dot{\mathbf{x}}_{\mathrm{R}}\right\|^{2}=N_{t}$, which is associated to binary phase shift keying (BPSK), quadrature phase shift keying (QPSK) and Phase Shift Keying (PSK) modulations. In order to overcome this challenge, the problem is reformulated as a convex optimization problem by relaxing the constraint as

$$
\check{\mathbf{x}}_{\mathrm{R}, \mathrm{ML}}^{(2)}=\arg \max _{\substack{\mathbf{x}_{\mathrm{R}} \in \mathbb{R}^{2 N_{t} \times 1} \\\left\|\dot{\mathbf{x}}_{\mathrm{R}}\right\|^{2} \leq N_{t}}} \sum_{i=1}^{2} \sum_{n=1}^{N_{r}} \Psi\left(\sqrt{2 \rho} \tilde{h}_{\mathrm{R}_{n, i}}^{T} \dot{\mathbf{x}}_{\mathrm{R}}\right) .
$$

After solving (2-36), the BS needs to perform normalization followed by symbol-by-symbol detection. The normalization of $\check{\mathbf{x}}_{\mathrm{R}, \mathrm{ML}}^{(2)}$ is given by

$$
\overline{\mathbf{x}}_{\mathrm{R}, \mathrm{ML}}=\sqrt{N_{t}} \frac{\check{\mathbf{x}}_{\mathrm{R}, \mathrm{ML}}^{(2)}}{\| \check{\mathbf{x}}_{\mathrm{R}, \mathrm{ML} \|_{2}^{(2)}}^{2}}
$$

Finally, by letting $\bar{x}_{\mathrm{R}, \mathrm{ML}, k}$ be the $k$ th element of $\overline{\mathbf{x}}_{\mathrm{R}, \mathrm{ML}}$, we get the $\mathrm{nML}$ symbol-by-symbol detection stated as

$$
\hat{x}_{\mathrm{nML}, k}=\arg \min _{\dot{x} \in \mathcal{S}}\left|\left(\bar{x}_{\mathrm{R}, \mathrm{ML}, k}+j \bar{x}_{\mathrm{R}, \mathrm{ML}, N_{t}+k}\right)-\dot{x}\right| .
$$

The authors in [15] also proposed a two-stage nML detector which has performance improvement in comparison with the one described so far. Based on the output of the one-stage nML detector the number of candidate transmit vectors is reduced. Defining the candidate set of the $k$ th element as

$$
x_{k}=\left\{\dot{x} \in \mathcal{S} \mid \frac{\left|\bar{x}_{\mathrm{R}, \mathrm{ML}, k}+j \bar{x}_{\mathrm{R}, \mathrm{ML}, N_{t}+k}-\dot{x}\right|}{\left|\left(\bar{x}_{\mathrm{R}, \mathrm{ML}, k}+j \bar{x}_{\mathrm{R}, \mathrm{ML}, N_{t}+k}\right)-\hat{x}_{\mathrm{nML}, k}\right|}<c\right\},
$$

where $c$ is a constant that controls the size of $X_{k}$. By setting $c$ with a proper value, it is possible to effectively improve the detection performance. The reduced candidate set of the transmit vectors can be defined as $X=$ $\left\{\left[\check{x}_{1} \cdots \check{x}_{N_{t}}\right]^{T} \mid \check{x}_{k} \in X_{k}, \forall k\right\}$. And, the real-form vector can be stated as $X_{\mathrm{R}}=$ $\left\{\left[\operatorname{Re}(\dot{\mathbf{x}})^{T} \operatorname{Im}(\dot{\mathbf{x}})^{T}\right]^{T} \mid \dot{\mathbf{x}} \in \mathcal{X}\right\}$. With this, the two-stage nML detector can be 
defined as

$$
\hat{\mathbf{x}}_{\mathrm{R}, \text { two-nML }}=\arg \max _{\hat{\mathbf{x}}_{\mathrm{R}} \in \mathcal{X}_{\mathrm{R}}} \prod_{i=1}^{2} \prod_{n=1}^{N_{r}} \Psi\left(\sqrt{2 \rho} \tilde{h}_{\mathrm{R}_{n, i}}^{T} \dot{\mathbf{x}}_{\mathrm{R}}\right) .
$$

The concept of the two-stage nML detector is similar to the sphere decoding, which exploits a reduced search space.

\subsection{4}

\section{One-Bit Spatial Sigma-Delta Detector}

Different from the approaches aforementioned, the 1-bit spatial sigma delta approach relies on spatial oversampling. Some characteristics of the $\Sigma \Delta$ approach are: it includes quantization error feedback loops and uses a modified Bussgang solution because the standard one leads to a linear model that is inconsistent with the corresponding hardware implementation.

The authors in $[24,25]$ have used spatial oversampling by employing a one-bit $\Sigma \Delta$ sampling scheme. In order to reduce the effects of the noise quantization with low-pass spatial filters or beamformers, it is shifted to higher spatial frequencies. Moreover, with optimal quantizer output settings the power of the quantization noise does not grow with the number of antennas (despite the fact that is propagated from one antenna to the next), but it converges to a constant value of approximately 1.33 times the input power.

Simulation results of the papers mentioned in this section have shown large performance gains on channel estimation and signal detection offered by this approach.

\section{4}

\section{Sum Rate Analysis}

\subsection{1}

\section{Data Transmission with LMMSE Receiver}

In this subsection it is considered that in the data transmission stage the $N_{t}$ users simultaneously transmit their data symbols represented by the vector $\mathbf{x}$ to the BS. After processed by the comparators, the quantized signal can be expressed as

$$
\mathbf{y}_{\mathcal{Q}_{d}}=\mathcal{Q}\left(\mathbf{y}_{d}\right)=\mathcal{Q}\left(\mathbf{H x}+\mathbf{n}_{d}\right)=\mathbf{A}_{d} \mathbf{H} \mathbf{x}+\mathbf{A}_{d} \mathbf{n}_{d}+\mathbf{n}_{q, d}
$$

where the same definitions from Section 2.2 apply, but with the subscript $p$ replaced by $d$, since we changed from the pilots to the data transmission stage. Then, the LRA-LMMSE channel estimate in (2-13) is used to compute a linear receiver which provides an estimate of the data symbols transmitted from the $N_{t}$ users. Thereby, we obtain 


$$
\begin{aligned}
\hat{\mathbf{x}} & =\mathbf{G y}_{\mathcal{Q}_{d}}=\mathbf{G}\left(\mathbf{A}_{d} \mathbf{H} \mathbf{x}+\mathbf{A}_{d} \mathbf{n}_{d}+\mathbf{n}_{q, d}\right) \\
& =\mathbf{G A}_{d}\left(\hat{\mathbf{H}}_{\mathrm{LRA}-\mathrm{LMMSE}} \mathbf{x}+\mathcal{E} \mathbf{x}\right)+\mathbf{G} \mathbf{A}_{d} \mathbf{n}_{d}+\mathbf{G n}_{q, d}
\end{aligned}
$$

where $\hat{\mathbf{H}}_{\text {LRA-Lmmse }}=\operatorname{unvec}\left(\hat{\mathbf{h}}_{\text {LRA-LMmSE }}\right)$. Finally, $\mathcal{E}=\mathbf{H}-\hat{\mathbf{H}}_{\text {LRA-LMmSE }}$ is the channel estimation error. Then, the $k$ th element represents an estimate of the signal of the $k$ th user, similarly as in [16], with $k \in\left[1, N_{t}\right]$, which reads

$$
\hat{\mathbf{x}}_{k}=\underbrace{\mathbf{g}_{k}^{T} \mathbf{A}_{d} \hat{\mathbf{h}}_{k} \mathbf{x}_{k}}_{\text {desired signal }}+\underbrace{\mathbf{g}_{k}^{T} \sum_{i \neq k}^{K} \mathbf{A}_{d} \hat{\mathbf{h}}_{i} \mathbf{x}_{i}}_{\text {user interference }}+\underbrace{\mathbf{g}_{k}^{T} \sum_{i=1}^{K} \mathbf{A}_{d} \boldsymbol{\varepsilon}_{i} \mathbf{x}_{i}}_{\text {channel estimation error }}+\underbrace{\mathbf{g}_{k}^{T} \mathbf{A}_{d} \mathbf{n}_{d}}_{\text {AWGN noise }}+\underbrace{\mathbf{g}_{k}^{T} \mathbf{n}_{q, d}}_{\text {quant. noise }}
$$

where $\mathbf{g}_{k}^{T}$ is the $k$ th row of $\mathbf{G}$ and $\hat{\mathbf{h}}_{k}$ is the $k$ th column of $\hat{\mathbf{H}}_{\text {LRA-LMmSE. }}$ Moreover, $\boldsymbol{\varepsilon}_{i}$ is the $i$ th column of the matrix $\mathcal{E}$.

\subsection{2}

\section{Lower Bounding the Sum Rate}

Since the Gaussian noise case corresponds to the worst case scenario, we can find a lower bound for the achievable rate by interpreting the quantization noise as Gaussian, with an equivalent noise covariance matrix [33]. In this regard, the equivalent noise covariance matrix is given by

$$
\mathbf{C}_{\mathbf{n}_{q, d}}=\mathbf{C}_{\mathbf{y}_{\mathcal{Q}_{d}}}-\mathbf{A}_{d} \mathbf{C}_{\mathbf{y}_{d}} \mathbf{A}_{d}^{H}
$$

where $\mathbf{C}_{\mathbf{y}_{\mathcal{Q}_{d}}}$ is the auto-correlation matrix of the quantized data signal, like in (2-30), and $\mathbf{C}_{\mathbf{y}_{d}}$ is the auto-correlation matrix of the received data signal, as calculated in (2-26). Using this approach, the ergodic achievable rate of user $k$ relates to the differential entropy and is lower bounded by [16]

$$
I_{k}=E\left[\log _{2}\left(1+\frac{\left|\mathbf{d}_{k} \hat{\mathbf{h}}_{k}\right|^{2}}{\sum_{i \neq k}^{K}\left|\mathbf{d}_{k} \hat{\mathbf{h}}_{i}\right|^{2}+\sum_{i=1}^{K}\left|\mathbf{d}_{k} \boldsymbol{\varepsilon}_{i}\right|^{2}+\sigma_{n}^{2}\left\|\mathbf{d}_{k}\right\|_{2}^{2}+\mathbf{g}_{k}^{T} \mathbf{C}_{\mathbf{n}_{q, d}} \mathbf{g}_{k}}\right)\right],
$$

where $\mathbf{d}_{k}=\mathbf{g}_{k}^{T} \mathbf{A}_{d}$ and the expectation operator is taken with respect to channel realizations and channel estimation realizations. Finally, the sum rate is lowerbounded by $\sum_{k=1}^{K} I_{k}$.

\section{5}

\section{Summary}

In this chapter we have presented a review on the technical background of this thesis. Some of the basic knowledge about MIMO systems was illustrated, 
including pilot-based channel estimation, detection and sum rate analysis approaches. 


\section{3}

\section{System Model}

In this chapter, the system model for the proposed MIMO receiver architecture with 1-bit quantization which has an additional comparator network is illustrated. Next, the design of the comparator network, including two types of networks: fully and partially connected, is introduced and described.

\section{1}

\section{Proposed Comparator Network-aided MIMO Receiver}

The general system model for the proposed comparator network-aided MIMO system with 1-bit ADCs at the receiver is illustrated with blocks in Fig. 3.1, where the received signal $\mathbf{y}$ for the uplink single-cell MIMO system with $N_{t}$ single-antenna users and $N_{r}$ receive antennas is written as

$$
\mathbf{y}=\mathbf{H x}+\mathbf{n} .
$$

The vector $\mathbf{x}$ contains complex transmit symbols of the $N_{t}$ users which have unit power normalization, $\mathbf{H} \in \mathbb{C}^{N_{r} \times N_{t}}$ is the channel matrix and $\mathbf{n} \in \mathbb{C}^{N_{r} \times 1}$ is the noise vector where each element has a variance $\sigma_{n}^{2}$.

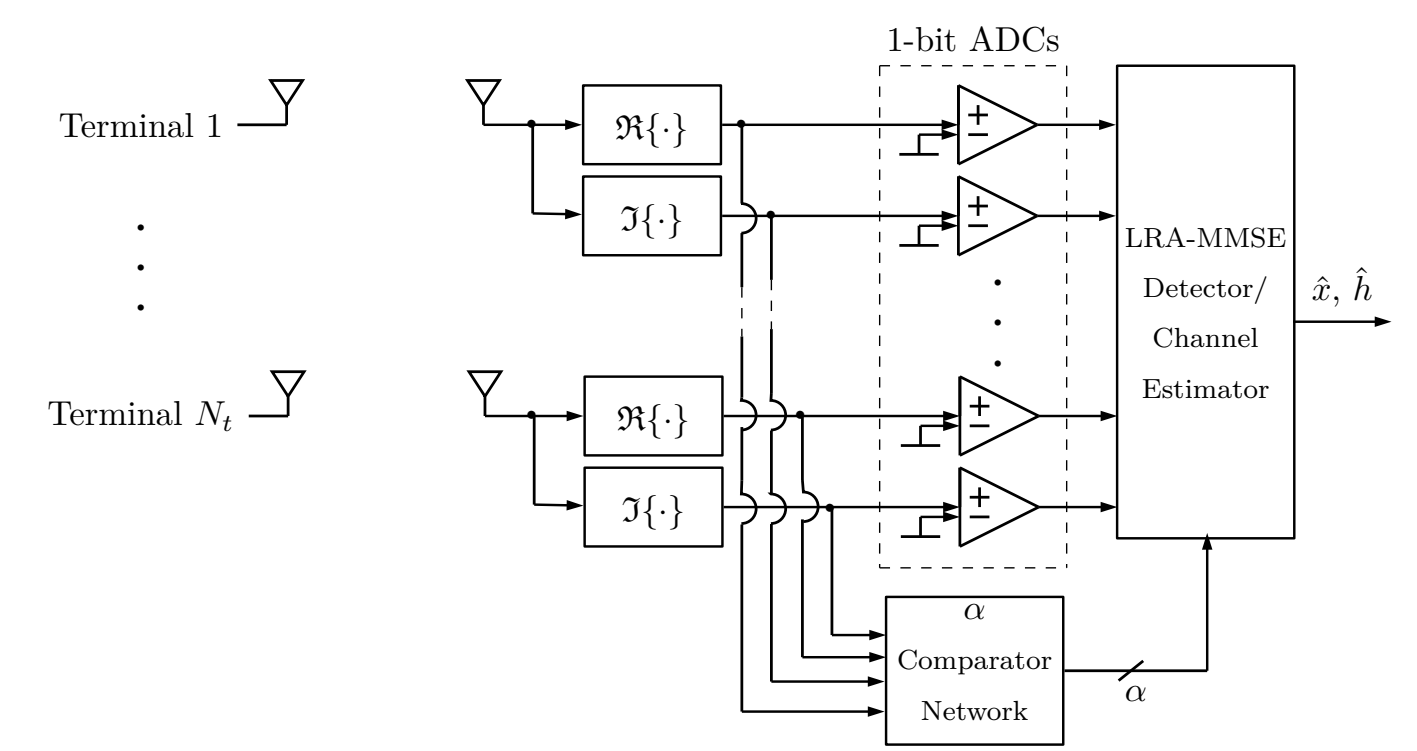

Figure 3.1: System model of MU-MIMO with 1-bit ADCs and an additional comparator network. 
Using the transformation from a complex into a real-valued system, we obtain

$$
\left[\begin{array}{c}
\mathfrak{R}\{\mathbf{y}\} \\
\mathfrak{I}\{\mathbf{y}\}
\end{array}\right]=\left[\begin{array}{cc}
\mathfrak{R}\{\mathbf{H}\} & -\mathfrak{I}\{\mathbf{H}\} \\
\mathfrak{I}\{\mathbf{H}\} & \mathfrak{R}\{\mathbf{H}\}
\end{array}\right]\left[\begin{array}{c}
\mathfrak{R}\{\mathbf{x}\} \\
\mathfrak{I}\{\mathbf{x}\}
\end{array}\right]+\left[\begin{array}{c}
\mathfrak{R}\{\mathbf{n}\} \\
\mathfrak{I}\{\mathbf{n}\}
\end{array}\right],
$$

where $\mathfrak{R}\{\cdot\}$ and $\mathfrak{I}\{\cdot\}$ denote the real and imaginary parts, respectively. A more compact notation for equation $(3-2)$ reads as

$$
\mathbf{y}_{\mathrm{R}}=\mathbf{H}_{\mathrm{R}} \mathbf{x}_{\mathrm{R}}+\mathbf{n}_{\mathrm{R}} \text {. }
$$

The received signal is then forwarded to the 1-bit ADCs and the comparator network (shown in Fig. 3.2). Each comparator compares two received signals and quantizes the difference as $\left\{ \pm \frac{1}{\sqrt{2}}\right\}$.

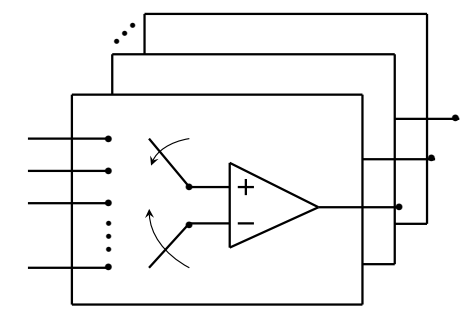

Figure 3.2: Insight of the comparator network.

Letting $\mathcal{Q}(\cdot)$ represent the 1-bit quantization, the output of the ADCs and the comparator network is described by

$$
\mathbf{z}_{\mathcal{Q}}=\mathcal{Q}\left(\left[\begin{array}{c}
\mathbf{y}_{\mathrm{R}} \\
\mathbf{B}^{\prime} \mathbf{y}_{\mathrm{R}}
\end{array}\right]\right)=\mathcal{Q}\left(\left[\begin{array}{c}
\mathbf{I}_{2 N_{r}} \\
\mathbf{B}^{\prime}
\end{array}\right] \mathbf{y}_{\mathrm{R}}\right)
$$

where $\mathbf{B}^{\prime} \in \mathbb{R}^{\alpha \times 2 N_{r}}$ refers to the comparator network and has the form

$$
\mathbf{B}^{\prime}=\left[\begin{array}{ll}
\mathbf{B}_{\mathrm{R}}^{\prime} & \mathbf{B}_{\mathrm{I}}^{\prime}
\end{array}\right]=\left[\begin{array}{cccccc}
1 & -1 & 0 & 0 & \cdots & 0 \\
-1 & 0 & 1 & 0 & \cdots & 0 \\
0 & 0 & 0 & -1 & \cdots & 1 \\
\vdots & \vdots & \vdots & \vdots & \vdots & \vdots \\
0 & 1 & 0 & 0 & \cdots & -1
\end{array}\right]
$$

In each row of $\mathbf{B}^{\prime}$, there is only one pair of 1 and -1 and the remaining entries are zeros. With $\mathbf{B}=\left[\mathbf{I}_{2 N_{r}} ; \mathbf{B}^{\prime}\right],(3-4)$ reads as

$$
\mathbf{z}_{\mathcal{Q}}=\mathcal{Q}\left(\mathbf{z}_{\mathrm{R}}\right)=\mathcal{Q}\left(\mathbf{B} \mathbf{y}_{\mathrm{R}}\right)
$$

Besides the introduction of the novel MIMO receiver architecture, the contribution of the present study relies on the development of MIMO detection and channel estimation algorithms for the proposed system. Moreover, a sum rate analysis is presented using the aforementioned receive processing. 


\section{2}

\section{Comparator Network Design}

The matrix design of the comparator network in (3-5) is illustrated in this section. Two types of networks are considered, namely, fully and partially connected networks.

\subsection{1}

\section{Fully Connected Network}

In this network, every two of the received signals are compared, meaning that the number of comparators needed is $\alpha_{f}=\left(\begin{array}{c}2 N_{r} \\ 2\end{array}\right)=N_{r}\left(2 N_{r}-1\right)$. This reasoning is illustrated in Fig. 3.3.

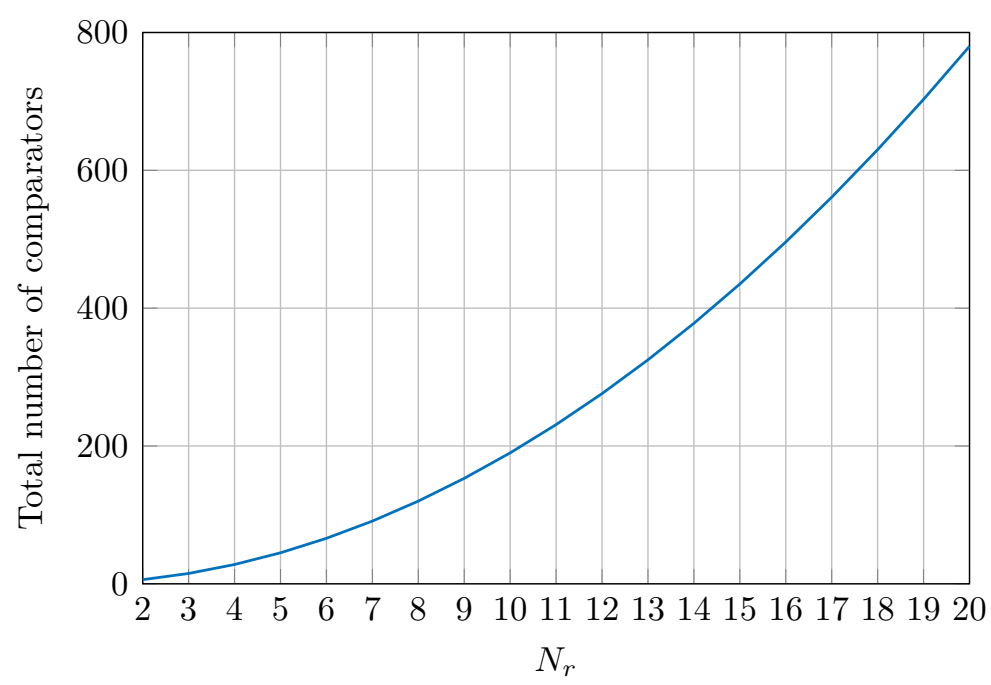

Figure 3.3: Number of comparators required in the fully connected network.

For instance, if we consider a system with $N_{r}=2$ receive antennas, $\alpha_{f}=\left(\begin{array}{l}4 \\ 2\end{array}\right)=6$ comparators are needed in this network. Then, the corresponding matrix $\mathbf{B}^{\prime}$ is described by

$$
\mathbf{B}^{\prime}=\left[\begin{array}{cccc}
1 & -1 & 0 & 0 \\
1 & 0 & -1 & 0 \\
1 & 0 & 0 & -1 \\
0 & 1 & -1 & 0 \\
0 & 1 & 0 & -1 \\
0 & 0 & 1 & -1
\end{array}\right]
$$

The main drawback of the fully connected network is the massive use of the comparators, where the number of comparators $\alpha_{f}$ is approximately proportional to the square of the number of receive antennas $N_{r}$. Therefore, in large-scale MIMO systems a much larger number of comparators will be required. 


\subsection{2}

\section{Partially Connected Network}

In order to increase the usage efficiency of the comparators, the partially connected network is proposed, where the number of utilized comparators $\alpha_{p}$ is only a small fraction of what is required for the fully connected network. For the same scenario described in the last subsection, one possibility for the corresponding matrix $\mathbf{B}_{\alpha_{p}}^{\prime}$ can be described by

$$
\mathbf{B}_{\alpha_{p}}^{\prime}=\left[\begin{array}{cccc}
1 & -1 & 0 & 0 \\
1 & 0 & -1 & 0 \\
1 & 0 & 0 & -1 \\
0 & 1 & -1 & 0
\end{array}\right]
$$

In this section, two types of network design are considered, random and greedy search based. The former approach criteria is to randomly select $\alpha_{p}$ out of $\alpha_{f}$ comparators $\left(\alpha_{p}<<\alpha_{f}\right)$, while the selection criterion of the latter is the MSE when using an LRA-LMMSE filter which will be developed in Chapter 5.

\section{3}

\section{Summary}

In this chapter, the proposed system model was illustrated and the design of the comparator network was described. Furthermore, two types of networks are introduced, namely, fully and partially connected networks. The partially connected network refers to comparator networks of $\alpha_{p}$ comparators with input signals from any two antennas in terms of real or imaginary parts. The fully connected network refers to comparator networks where all possible combinations are considered, meaning $\alpha_{f}$ comparators. 


\section{4 \\ Linear Channel Estimation}

In this chapter, we develop an LRA-LMMSE channel estimator based on the Bussgang theorem for the proposed comparator network-aided MIMO systems with 1-bit ADCs at the receiver. Additionaly, the mean-squared error of the channel estimate is derived. At last, numerical results of the developed schemes, are presented.

\section{1}

\section{Pilot-Based Channel Estimation}

A common technique for channel estimation is to let the users transmit orthogonal pilot sequences of $\tau$ pilot symbols and evaluate the effect of the channel on these symbols at the BS. During the training phase, all the terminals simultaneously transmit these sequences, which yields

$$
\mathbf{Y}_{p}=\mathbf{H} \Phi^{T}+\mathbf{N}_{p}
$$

where $\mathbf{Y}_{p} \in \mathbb{C}^{N_{r} \times \tau}$ is the matrix containing the unquantized received signal, $\boldsymbol{\Phi} \in \mathbb{C}^{\tau \times N_{t}}$ is the matrix of pilot symbols and $\mathbf{N}_{p}$ is the noise matrix. Vectorizing the received signal yields

$$
\operatorname{vec}\left(\mathbf{Y}_{p}\right)=\mathbf{y}_{p}=\tilde{\mathbf{\Phi}} \mathbf{h}+\mathbf{n}_{p}
$$

where $\tilde{\boldsymbol{\Phi}}=\left(\boldsymbol{\Phi} \otimes \mathbf{I}_{N_{r}}\right), \mathbf{h}=\operatorname{vec}(\mathbf{H})$ and $\mathbf{n}_{p}=\operatorname{vec}\left(\mathbf{N}_{p}\right)$. However, due to the addition of the comparator network, we need to consider a real-valued representation of the system. In this case, the received signal $\mathbf{y}_{\mathrm{R}_{p}}$ is defined by

$$
\begin{aligned}
\mathbf{y}_{\mathrm{R}_{p}} & =\left[\begin{array}{cc}
\mathfrak{R}\{\tilde{\boldsymbol{\Phi}}\} & -\mathfrak{I}\{\tilde{\boldsymbol{\Phi}}\} \\
\mathfrak{I}\{\tilde{\boldsymbol{\Phi}}\} & \mathfrak{R}\{\tilde{\boldsymbol{\Phi}}\}
\end{array}\right]\left[\begin{array}{c}
\mathfrak{R}\{\mathbf{h}\} \\
\mathfrak{I}\{\mathbf{h}\}
\end{array}\right]+\left[\begin{array}{c}
\mathfrak{R}\left\{\mathbf{n}_{p}\right\} \\
\mathfrak{I}\left\{\mathbf{n}_{p}\right\}
\end{array}\right] \\
& =\tilde{\boldsymbol{\Phi}}_{\mathrm{R}} \mathbf{h}_{\mathrm{R}}+\mathbf{n}_{\mathrm{R}_{p}},
\end{aligned}
$$

where $\mathbf{y}_{\mathrm{R}_{p}} \in \mathbb{R}^{\tau\left(2 N_{r}\right) \times 1}$ is the real-valued received signal vector. Then, when we multiply equation (4-3) with an effective comparator network matrix $\mathbf{B}_{\text {eff, we }}$ get

$$
\mathbf{z}_{\mathrm{R}_{p}}=\mathbf{B}_{\text {eff }} \mathbf{y}_{\mathrm{R}_{p}}=\mathbf{B}_{\mathrm{eff}} \tilde{\boldsymbol{\Phi}}_{\mathrm{R}} \mathbf{h}_{\mathrm{R}}+\mathbf{B}_{\mathrm{eff}} \mathbf{n}_{\mathrm{R}_{p}}
$$

where $\mathbf{B}_{\text {eff }} \in \mathbb{R}^{\tau\left(2 N_{r}+\alpha\right) \times \tau\left(2 N_{r}\right)}$ is described by 


$$
\mathbf{B}_{\mathrm{eff}}=\left[\begin{array}{c}
\mathbf{I}_{\tau\left(2 N_{r}\right)} \\
\mathbf{B}_{\mathrm{eff}}^{\prime}
\end{array}\right]=\left[\begin{array}{c}
\mathbf{I}_{\tau\left(2 N_{r}\right)} \\
{\left[\begin{array}{ll}
\mathbf{B}_{\mathrm{eff}, \mathrm{R}}^{\prime} & \mathbf{B}_{\mathrm{eff}, \mathrm{I}}^{\prime}
\end{array}\right]}
\end{array}\right],
$$

where $\mathbf{B}_{\text {eff, } \mathrm{R}}^{\prime}=\left(\mathbf{B}_{\mathrm{R}}^{\prime} \otimes \mathbf{I}_{\tau}\right)$ and $\mathbf{B}_{\text {eff, I }}^{\prime}=\left(\mathbf{B}_{\mathrm{I}}^{\prime} \otimes \mathbf{I}_{\tau}\right)$, with $\mathbf{B}_{\mathrm{R}}^{\prime}$ and $\mathbf{B}_{\mathrm{I}}^{\prime}$ denoting respectively the parts of the matrix $\mathbf{B}^{\prime}$ which are associated to the real and imaginary parts of the received signal, as shown in (3-5). After being processed by the comparators, the quantized signal can be expressed as

$$
\mathbf{z}_{\mathcal{Q}_{p}}=\mathcal{Q}\left(\mathbf{z}_{\mathrm{R}_{p}}\right)=\hat{\boldsymbol{\Phi}}_{\mathrm{R}} \mathbf{h}_{\mathrm{R}}+\tilde{\mathbf{n}}_{\mathrm{R}_{p}}
$$

where the right hand side corresponds to a linear model that relies on the Bussgang decomposition approach. In this context, the linear model involves the matrix $\hat{\mathbf{\Phi}}_{\mathrm{R}}=\mathbf{A}_{\mathrm{R}_{p}} \mathbf{B}_{\text {eff }} \tilde{\mathbf{\Phi}}_{\mathrm{R}}$ and the effective noise vector $\tilde{\mathbf{n}}_{\mathrm{R}_{p}}=$ $\mathbf{A}_{\mathrm{R}_{p}} \mathbf{B}_{\text {eff }} \mathbf{n}_{\mathrm{R}_{p}}+\mathbf{n}_{q, p}$, with $\mathbf{A}_{\mathrm{R}_{p}}$ being a well chosen square matrix and $\mathbf{n}_{q, p}$ being the quantization noise. The Bussgang-based linear operator $\mathbf{A}_{\mathrm{R}_{p}}$ is chosen to make the quantization noise uncorrelated with $\mathbf{z}_{\mathrm{R}_{p}}$ [33], and is given by

$$
\mathbf{A}_{\mathrm{R}_{p}}=\mathbf{C}_{\mathbf{z}_{\mathrm{R}_{p} \mathbf{z}_{\mathcal{Q}} p}^{H}}^{H} \mathbf{C}_{\mathbf{z}_{\mathrm{R}_{p}}}^{-1}=\sqrt{\frac{2}{\pi}} \mathbf{K}_{\mathrm{R}_{p}},
$$

with $\mathbf{K}_{\mathrm{R}_{p}}=\operatorname{diag}\left(\mathbf{C}_{\mathbf{z}_{\mathrm{R}_{p}}}\right)^{-\frac{1}{2}}$, where $\mathbf{C}_{\mathbf{z}_{\mathrm{R}_{p}} \mathbf{z}_{\mathcal{Q}_{p}}}$ denotes the cross-correlation matrix between the received signal $\mathbf{z}_{\mathrm{R}_{p}}$ and its quantized signal $\mathbf{z}_{\mathcal{Q}_{p}}$ is given by

$$
\mathbf{C}_{\mathbf{z}_{\mathrm{R}_{p} \mathbf{z}_{\mathcal{Q}_{p}}}}=\sqrt{\frac{2}{\pi}} \mathbf{K}_{\mathrm{R}_{p}} \mathbf{C}_{\mathbf{z}_{\mathrm{R}_{p}}}=\mathbf{A}_{\mathrm{R}_{p}} \mathbf{C}_{\mathbf{z}_{\mathrm{R}_{p}}}
$$

and $\mathbf{C}_{\mathbf{z}_{\mathrm{R}_{p}}}$ denotes the auto-correlation matrix of $\mathbf{z}_{\mathrm{R}_{p}}$

$$
\begin{aligned}
\mathbf{C}_{\mathbf{z}_{\mathrm{R} p}} & =\mathbf{B}_{\text {eff }} \tilde{\boldsymbol{\Phi}}_{\mathrm{R}} \mathbf{R}_{\mathbf{h}_{\mathrm{R}}} \tilde{\boldsymbol{\Phi}}_{\mathrm{R}}^{T} \mathbf{B}_{\mathrm{eff}}^{T}+\mathbf{B}_{\mathrm{eff}} \mathbf{C}_{\mathbf{n}_{\mathrm{R}_{\mathbf{p}}}} \mathbf{B}_{\mathrm{eff}}^{T} \\
& =\frac{1}{2} \mathbf{B}_{\mathrm{eff}} \tilde{\boldsymbol{\Phi}}_{\mathrm{R}} \tilde{\boldsymbol{\Phi}}_{\mathrm{R}}^{T} \mathbf{B}_{\mathrm{eff}}^{T}+\frac{\sigma_{n}^{2}}{2} \mathbf{B}_{\mathrm{eff}} \mathbf{B}_{\mathrm{eff}}^{T},
\end{aligned}
$$

where it is assumed that $\mathbf{R}_{\mathbf{h}_{\mathrm{R}}}=E\left[\mathbf{h}_{\mathrm{R}} \mathbf{h}_{\mathrm{R}}^{T}\right]=\frac{1}{2} \mathbf{I}_{2 N_{t} N_{r}}$ and $\mathbf{C}_{\mathbf{n}_{\mathrm{R}_{\mathbf{p}}}}=$ $E\left[\mathbf{n}_{\mathrm{R}_{p}} \mathbf{n}_{\mathrm{R}_{p}}^{T}\right]=\frac{\sigma_{n}^{2}}{2} \mathbf{I}_{\tau\left(2 N_{r}\right)}$. Note that the factor $\frac{1}{2}$ is due to the real-valued notation of the system.

Derivation: See Appendix A.1.

\section{2}

\section{Proposed LRA-LMMSE Channel Estimator}

Based on the statistically equivalent linear model in (4-6), the LRALMMSE optimal filter can be obtained through the optimization problem formulated as 


$$
\begin{aligned}
\mathbf{W}_{\mathrm{R}, \text { LRA-LMMSE }} & =\arg \min _{\mathbf{W}} E\left[\left\|\mathbf{h}_{\mathrm{R}}-\mathbf{W}_{\mathbf{z}_{\mathcal{Q}}}\right\|_{2}^{2}\right] \\
& =\mathbf{R}_{\mathbf{h}_{\mathrm{R}}} \hat{\boldsymbol{\Phi}}_{\mathrm{R}}^{T} \mathbf{C}_{\mathbf{z}_{\mathcal{Q} p}}^{-1}=\frac{1}{2} \hat{\boldsymbol{\Phi}}_{\mathrm{R}}^{T} \mathbf{C}_{\mathbf{z}_{\mathcal{Q} p}}^{-1},
\end{aligned}
$$

where the auto-correlation of the quantized signal is calculated as [34]

$$
\mathbf{C}_{\mathbf{z}_{\mathcal{Q}_{p}}}=\frac{2}{\pi} \sin ^{-1}\left(\mathbf{K}_{\mathrm{R}_{p}} \mathbf{C}_{\mathbf{z}_{\mathrm{R} p}} \mathbf{K}_{\mathrm{R}_{p}}\right) \text {. }
$$

The resulting LRA-LMMSE channel estimator corresponds to the linear operation

$$
\hat{\mathbf{h}}_{\mathrm{R}, \text { LRA-LMMSE }}=\left[\begin{array}{c}
\hat{\mathbf{h}}_{\mathrm{R}, \text { LRA-LMMSE, a }} \\
\hat{\mathbf{h}}_{\mathrm{R}, \text { LRA-LMMSE, } \mathrm{b}}
\end{array}\right]=\mathbf{R}_{\mathbf{h}_{\mathrm{R}}} \hat{\boldsymbol{\Phi}}_{\mathrm{R}}^{T} \mathbf{C}_{\mathbf{z}_{\mathcal{Q}_{p}}}^{-1} \mathbf{z}_{\mathcal{Q}_{p}}=\frac{1}{2} \hat{\boldsymbol{\Phi}}_{\mathrm{R}}^{T} \mathbf{C}_{\mathbf{z}_{\mathcal{Q}_{p}}}^{-1} \mathbf{z}_{\mathcal{Q}_{p}},
$$

where $\hat{\mathbf{h}}_{\mathrm{R} \text {, LRA-LMMSE, a }}$ is the first half of the channel estimate which corresponds to the real part and $\hat{\mathbf{h}}_{\mathrm{R}, \text { LRA-LMMSE, } \mathrm{b}}$ is the second half which corresponds to the imaginary part.

Derivation: See Appendix A.2.

\section{3}

\section{Mean-Squared Error of the Channel Estimate}

The MSE of the LRA-LMMSE channel estimate can be expressed as

$$
\begin{aligned}
\mathcal{M}_{\mathrm{R}, \text { LRA-MMSE }} & =E\left[\left\|\hat{\mathbf{h}}_{\mathrm{R}, \text { LRA-MMSE }}-\mathbf{h}_{\mathrm{R}}\right\|_{2}^{2}\right] \\
& =\operatorname{tr}\left(\mathbf{R}_{\mathbf{h}_{\mathrm{R}}}-\mathbf{R}_{\mathbf{h}_{\mathrm{R}}} \hat{\boldsymbol{\Phi}}_{\mathrm{R}}^{T} \mathbf{C}_{\mathbf{z}_{\mathcal{Q}_{p}}}^{-1} \hat{\mathbf{\Phi}}_{\mathrm{R}} \mathbf{R}_{\mathbf{h}_{\mathrm{R}}}\right) \\
& =\operatorname{tr}\left(\frac{1}{2} \mathbf{I}_{2 N_{t} N_{r}}-\frac{1}{4} \hat{\boldsymbol{\Phi}}_{\mathrm{R}}^{T} \mathbf{C}_{\mathbf{z}_{\mathcal{Q}_{p}}}^{-1} \hat{\mathbf{\Phi}}_{\mathrm{R}}\right) .
\end{aligned}
$$

Derivation: See Appendix A.3.

\section{4}

\section{Numerical Results}

In this section, an uplink single-cell 1-bit MIMO system with comparator network, $N_{t}=2$ and $N_{r}=4$ is considered. The SNR is defined as $10 \log \left(\frac{1}{\sigma_{n}^{2}}\right)$, which is the average receive SNR per user per antenna. The lines labeled as "Analytical Result" are obtained with (4-13) while the marks labeled as "Numerical Result" are obtained with the MSE of the simulated channel estimator in (4-12). In both experiments, the partially connected networks have $\alpha_{p}=2 N_{r}=8$ comparators, while the fully connected have $\alpha_{f}=\left(\begin{array}{c}2 N_{r} \\ 2\end{array}\right)=28$ comparators.

The MSE comparison between the LRA-LMMSE channel estimators with fully and partially connected comparator networks are shown in Fig. 4.1. The 


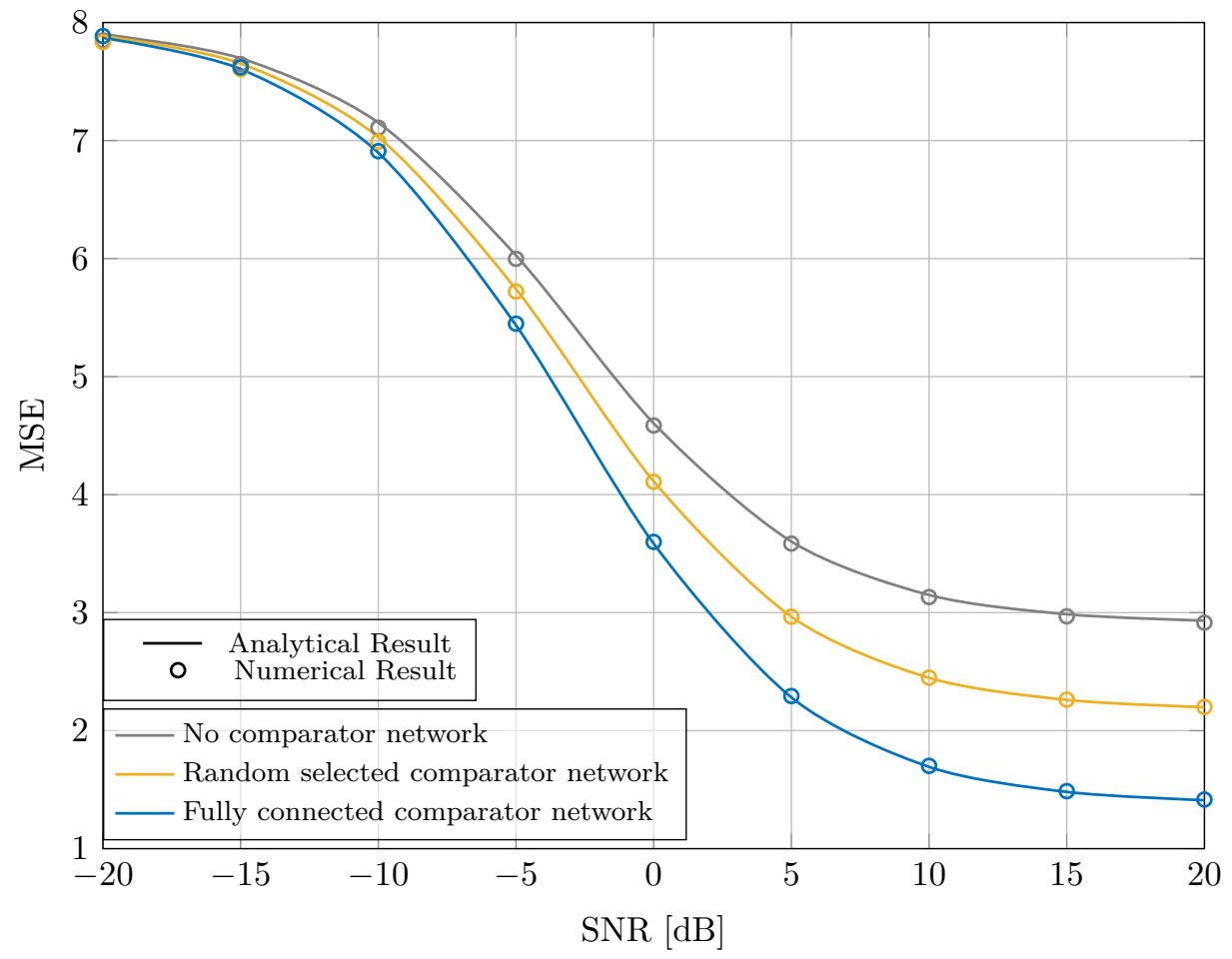

Figure 4.1: MSE comparisons of LRA-LMMSE channel estimators in $2 \times 4$ MIMO systems with $\tau=N_{t}=2$.

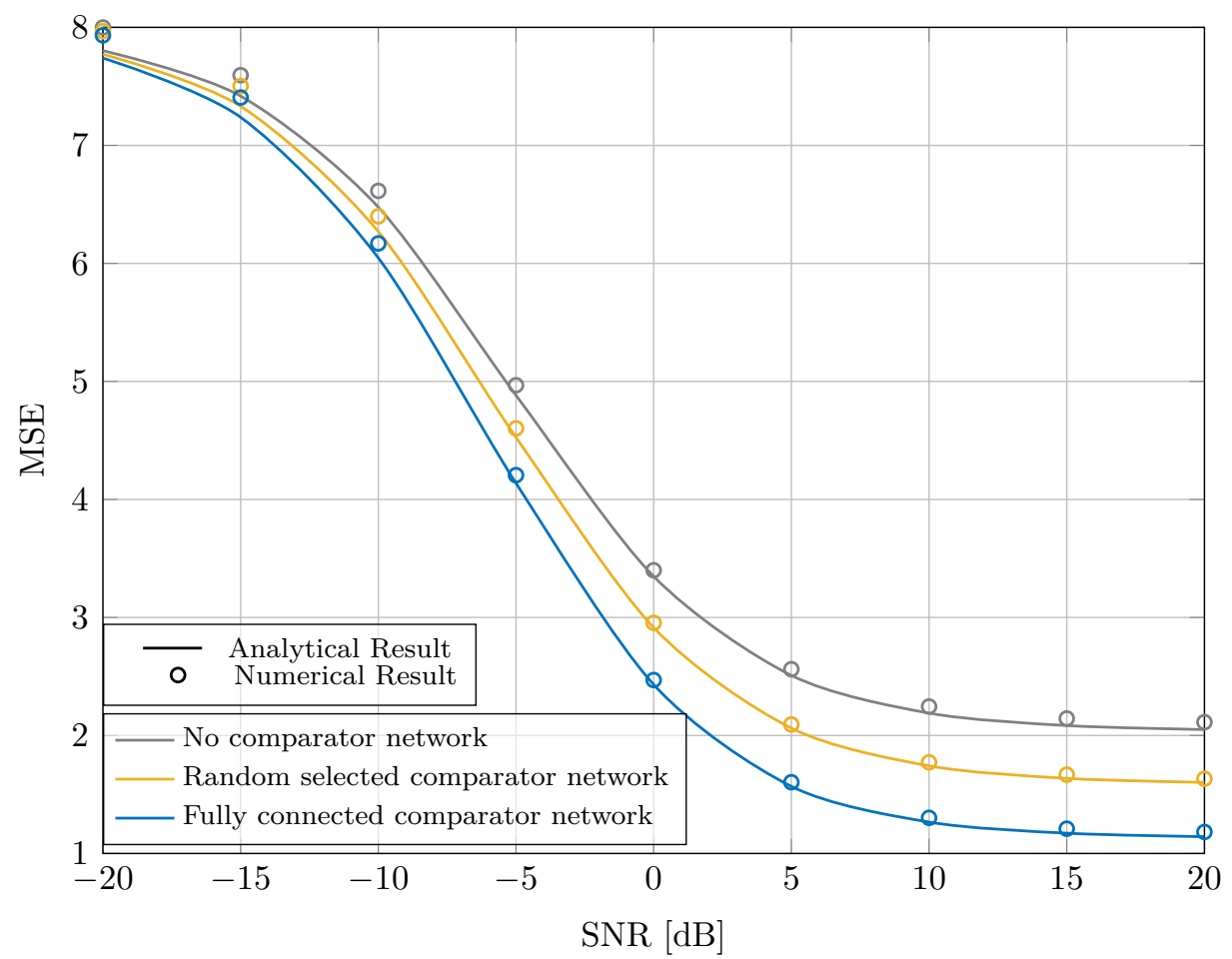

Figure 4.2: MSE comparisons of LRA-LMMSE channel estimators in $2 \times 4$ MIMO systems with $\tau=2 N_{t}=4$.

pilot sequences are column-wise orthogonal with length $\tau=N_{t}=2$, i.e., $\boldsymbol{\Phi}^{T} \boldsymbol{\Phi}=\tau \mathbf{I}_{N_{t}}$. The presented performance plots are obtained by taking the 
average over 2000 different channels and 2000 noise realizations per channel. The numerical and analytical results are aligned, which confirms the accuracy of the proposed model. As expected, the system with the fully connected method achieves the best MSE performance. However, it can be seen that the approach with the comparator network using random selected inputs is also beneficial in terms of MSE in comparison to the case without a comparator network.

The results in Fig. 4.2 were obtained by changing the size of the pilot sequences to $\tau=2 N_{t}=4$. The presented performance plots are obtained by taking the average over 700 different channels and 700 noise realizations per channel.

\section{5}

\section{Summary}

In this chapter, we have derived the LRA-LMMSE channel estimator for comparator network aided 1-bit MIMO systems. The pilot-based channel estimation for the real-valued system representation was proposed. The simulation results have shown a great performance gain for the proposed channel estimator with the additional comparator network. Moreover, the numerical simulations match with the corresponding analytical channel estimation performance in terms of the MSE, which corroborates the accuracy of the proposed model. 


\section{Linear Detection}

In this chapter, we develop the LRA-LMMSE detector based on the Bussgang theorem for the proposed comparator network-aided MIMO system with 1-bit ADCs at the receiver. Moreover, a robust version of the LRALMMSE detector is devised, which takes into account the channel estimation error statistics. In the sequel, we present the Greedy Search Algorithm for the optimization of the partially connected network. Then, the computational complexity, computational and hardware costs of the methods are calculated for comparison purposes. Finally, numerical results of the developed schemes, are individually and integrally illustrated.

\section{1}

\section{Proposed LRA-LMMSE Detector}

Based on the proposed system model in (3-6), the corresponding linear receiver to estimate the transmitted symbols is derived as follows. The optimization problem for getting the optimal linear receiver is formulated as

$$
\mathbf{G}_{\mathrm{R}, \text { LRA-MMSE }}=\arg \min _{\mathbf{G}_{\mathrm{R}}} E\left[\left\|\mathbf{x}_{\mathrm{R}}-\mathbf{G}_{\mathrm{R}}^{T} \mathbf{z}_{\mathcal{Q}}\right\|_{2}^{2}\right]
$$

where $\mathbf{G}_{\mathrm{R}} \in \mathbb{R}^{\left(2 N_{r}+\alpha\right) \times 2 N_{t}}$. The solution for this problem is given by

$$
\mathrm{G}_{\mathrm{R}, \text { LRA-MMSE }}=\mathbf{C}_{\mathbf{z}_{\mathcal{Q}}}^{-1} \mathbf{C}_{\mathbf{z}_{\mathcal{Q}} \mathbf{x}_{\mathrm{R}}}
$$

where the involved covariance matrix $\mathbf{C}_{\mathbf{z}_{\mathcal{Q}}}$ is calculated as [34]

$$
\mathbf{C}_{\mathbf{z}_{\mathcal{Q}}}=\frac{2}{\pi} \sin ^{-1}\left(\mathbf{K}_{\mathrm{R}} \mathbf{C}_{\mathbf{z}_{\mathrm{R}}} \mathbf{K}_{\mathrm{R}}\right)
$$

and the cross-correlation matrix $\mathbf{C}_{\mathbf{z}_{\mathcal{Q}} \mathbf{x}_{\mathrm{R}}}$ is based on the Bussgang theorem [36]

$$
\mathbf{C}_{\mathbf{z}_{\mathcal{Q}} \mathbf{x}_{\mathrm{R}}}=\sqrt{\frac{2}{\pi}} \mathbf{K}_{\mathrm{R}} \mathbf{C}_{\mathbf{z}_{\mathrm{R}} \mathbf{x}_{\mathrm{R}}}=\sqrt{\frac{2}{\pi}} \frac{1}{2} \mathbf{K}_{\mathrm{R}} \mathbf{B} \mathbf{H}_{\mathrm{R}}
$$

with $\mathbf{K}_{\mathrm{R}}=\operatorname{diag}\left(\mathbf{C}_{\mathbf{z}_{\mathrm{R}}}\right)^{-\frac{1}{2}}$ and the cross-correlation matrix between $\mathbf{z}_{\mathrm{R}}$ and $\mathbf{x}_{\mathrm{R}}$ is given by

$$
\mathbf{C}_{\mathbf{z}_{\mathrm{R}} \mathbf{x}_{\mathrm{R}}}=\mathrm{BH}_{\mathrm{R}} \mathbf{C}_{\mathbf{x}_{\mathrm{R}}}=\frac{1}{2} \mathrm{BH}_{\mathrm{R}}
$$


where due to the real-valued notation of the system $\mathbf{C}_{\mathbf{x}_{\mathrm{R}}}=E\left[\mathbf{x}_{\mathrm{R}} \mathbf{x}_{\mathrm{R}}^{T}\right]=\frac{1}{2} \mathbf{I}_{2 N_{t}}$. Derivation: See Appendix B.1.

Besides, the auto-correlation of $\mathbf{z}_{\mathrm{R}}$ is given by

$$
\begin{aligned}
\mathbf{C}_{\mathbf{z}_{\mathrm{R}}} & =\mathbf{B H}_{\mathrm{R}} \mathbf{C}_{\mathbf{x}_{\mathrm{R}}} \mathbf{H}_{\mathrm{R}}^{T} \mathbf{B}^{T}+\mathbf{B C}_{\mathbf{n}_{\mathrm{R}}} \mathbf{B}^{T} \\
& =\frac{1}{2} \mathbf{B H}_{\mathrm{R}} \mathbf{H}_{\mathrm{R}}^{T} \mathbf{B}^{T}+\frac{\sigma_{n}^{2}}{2} \mathbf{B B}^{T} .
\end{aligned}
$$

where due to the real-valued notation of the system $\mathbf{C}_{\mathbf{n}_{\mathrm{R}}}=E\left[\mathbf{n}_{\mathrm{R}} \mathbf{n}_{\mathrm{R}}^{T}\right]=\frac{\sigma_{n}^{2}}{2} \mathbf{I}_{2 N_{r}}$. Derivation: See Appendix B.2.

\section{2}

\section{Robust LRA-LMMSE Detector}

In this section, a robust LRA-MMSE detector is proposed, which takes into account the channel estimation and its corresponding error statistics. In this context, based on the channel estimation error $\varepsilon_{R}=\mathbf{h}_{R}-\hat{\mathbf{h}}_{R}$, LRA-LMMSE, we need to redefine the received signal as

$$
\begin{aligned}
\mathbf{y}_{\mathrm{R}_{r}} & =\left[\begin{array}{cc}
\mathfrak{R}\{\tilde{\mathbf{X}}\} & -\mathfrak{I}\{\tilde{\mathbf{X}}\} \\
\mathfrak{I}\{\tilde{\mathbf{X}}\} & \Re\{\tilde{\mathbf{X}}\}
\end{array}\right]\left[\begin{array}{c}
\mathfrak{R}\{\mathbf{h}\} \\
\mathfrak{I}\{\mathbf{h}\}
\end{array}\right]+\left[\begin{array}{c}
\mathfrak{R}\left\{\mathbf{n}_{d}\right\} \\
\mathfrak{I}\left\{\mathbf{n}_{d}\right\}
\end{array}\right] \\
& =\tilde{\mathbf{X}}_{\mathrm{R}} \mathbf{h}_{\mathrm{R}}+\mathbf{n}_{\mathrm{R}_{d}}=\tilde{\mathbf{X}}_{\mathrm{R}}\left(\hat{\mathbf{h}}_{\mathrm{R}, \text { LRA-LMMSE }}+\boldsymbol{\varepsilon}_{\mathrm{R}}\right)+\mathbf{n}_{\mathrm{R}_{d}} \\
& =\hat{\mathbf{H}}_{\mathrm{R}} \mathbf{x}_{\mathrm{R}}+\tilde{\mathbf{X}}_{\mathrm{R}} \boldsymbol{\varepsilon}_{\mathrm{R}}+\mathbf{n}_{\mathrm{R}_{d}},
\end{aligned}
$$

where $\mathbf{y}_{\mathrm{R}_{r}} \in \mathbb{R}^{2 N_{r} \times 1}$ and $\tilde{\mathbf{X}}=\left(\mathbf{x}^{T} \otimes \mathbf{I}_{N_{r}}\right) \in \mathbb{C}^{N_{r} \times N_{t} N_{r}}$. The subscripts $r$ and $d$ stand for robust and data, respectively. Moreover, $\hat{\mathbf{H}}_{\mathrm{R}} \in \mathbb{R}^{2 N_{r} \times 2 N_{t}}$ is the estimated channel matrix described by

$$
\begin{aligned}
& \hat{\mathbf{H}}_{\mathrm{R}}=\left[\begin{array}{cc}
\mathfrak{R}\{\hat{\mathbf{H}}\} & -\mathfrak{I}\{\hat{\mathbf{H}}\} \\
\mathfrak{I}\{\hat{\mathbf{H}}\} & \mathfrak{R}\{\hat{\mathbf{H}}\}
\end{array}\right] \text { with } \hat{\mathbf{H}}=\operatorname{unvec}(\hat{\mathbf{h}}) \\
& \text { and } \hat{\mathbf{h}}=\left[\hat{\mathbf{h}}_{\mathrm{R}, \text { LRA-LMmSE, a }}+j \hat{\mathbf{h}}_{\mathrm{R}, \text { LRA-LMMSE, } \mathrm{b}}\right],
\end{aligned}
$$

where $\hat{\mathbf{h}}_{\mathrm{R}, \text { LRA-LMMSE, a }}$ corresponds to the first half of the estimated channel $\hat{\mathbf{h}}_{\mathrm{R}, \text { LRA-LMMSE }}$ and $\hat{\mathbf{h}}_{\mathrm{R}, \text { LRA-LMMSE, } \mathrm{b}}$ to the second, as shown in (4-12). Therefore, the quantized signal for the robust detector is given by

$$
\begin{aligned}
\mathbf{z}_{\mathcal{Q}_{r}} & =\mathcal{Q}\left(\mathbf{z}_{\mathrm{R}_{r}}\right)=\mathcal{Q}\left(\mathbf{B} \mathbf{y}_{\mathrm{R}_{r}}\right)=\mathcal{Q}\left(\mathbf{B} \hat{\mathbf{H}}_{\mathrm{R}} \mathbf{x}_{\mathrm{R}}+\mathbf{B} \tilde{\mathbf{X}}_{\mathrm{R}} \boldsymbol{\varepsilon}_{\mathrm{R}}+\mathbf{B} \mathbf{n}_{\mathrm{R}_{d}}\right) \\
& =\mathbf{A}_{\mathrm{R}_{r}} \mathbf{B} \hat{\mathbf{H}}_{\mathrm{R}} \mathbf{x}_{\mathrm{R}}+\mathbf{A}_{\mathrm{R}_{r}} \mathbf{B} \tilde{\mathbf{X}}_{\mathrm{R}} \varepsilon_{\mathrm{R}}+\mathbf{A}_{\mathrm{R}_{r}} \mathbf{B n}_{\mathrm{R}_{d}}+\mathbf{n}_{\mathrm{R}_{q, d}},
\end{aligned}
$$

where $\mathbf{A}_{\mathrm{R}_{r}}$ is the Bussgang-based linear operator for the transmit data, similarly as calculated in (4-7). Then, the linear LRA-LMMSE filter is applied to $\mathbf{z}_{\mathcal{Q}_{r}}$, to obtain

$$
\hat{\mathbf{x}}_{\mathrm{R}}=\mathbf{G}_{\mathrm{R}}^{T} \mathbf{z}_{\mathcal{Q}_{r}},
$$


where the matrix $\mathbf{G}_{\mathrm{R}}$ is chosen to minimize the MSE between the transmitted symbol $\mathbf{x}_{\mathrm{R}}$ and the filter output, i.e.,

$$
\begin{aligned}
\tilde{\mathbf{G}}_{\mathrm{R}, \text { LRA-MMSE }} & =\arg \min _{\mathbf{G}_{\mathrm{R}}} E\left[\left\|\mathbf{x}_{\mathrm{R}}-\mathbf{G}_{\mathrm{R}}^{T} \mathbf{z}_{\mathcal{Q}_{r}}\right\|_{2}^{2}\right] \\
& =\arg \min _{\mathbf{G}_{\mathrm{R}}}-2 \operatorname{tr}\left(\mathbf{G}_{\mathrm{R}}^{T} \mathbf{C}_{\mathbf{z}_{\mathcal{Q}_{r}} \mathbf{x}_{\mathrm{R}}}\right)+\operatorname{tr}\left(\mathbf{G}_{\mathrm{R}}^{T} \mathbf{C}_{\mathbf{z}_{\mathcal{Q}_{r}}} \mathbf{G}_{\mathrm{R}}\right),
\end{aligned}
$$

where the second line describes an equivalent problem.

Derivation: See Appendix B.3.

By differentiating (5-11) with respect to $\mathbf{G}_{\mathrm{R}}^{T}$, the solution of the LRALMMSE receive filter is given by

$$
\tilde{\mathbf{G}}_{\mathrm{R}, \text { LRA-MMSE }}=\mathbf{C}_{\mathbf{z}_{\mathcal{Q}_{r}}}^{-1} \mathbf{C}_{\mathbf{z}_{\mathcal{Q}_{r}} \mathbf{x}_{\mathrm{R}}}
$$

where the covariance matrix is calculated as [34]

$$
\mathbf{C}_{\mathbf{z}_{\mathcal{Q}_{r}}}=\frac{2}{\pi} \sin ^{-1}\left(\mathbf{K}_{\mathrm{R}_{r}} \mathbf{C}_{\mathbf{z}_{\mathrm{R}_{r}}} \mathbf{K}_{\mathrm{R}_{r}}\right)
$$

and the cross-correlation matrix is based on the Bussgang theorem [36]

$$
\mathbf{C}_{\mathbf{z}_{\mathcal{Q}_{r}} \mathbf{x}_{\mathrm{R}}}=\sqrt{\frac{2}{\pi}} \mathbf{K}_{\mathrm{R}_{r}} \mathbf{C}_{\mathbf{z}_{\mathrm{R}_{r}} \mathbf{x}_{\mathrm{R}}}=\sqrt{\frac{2}{\pi}} \frac{1}{2} \mathbf{K}_{\mathrm{R}_{r}} \mathbf{B} \hat{\mathbf{H}}_{\mathrm{R}},
$$

with $\mathbf{K}_{\mathrm{R}_{r}}=\operatorname{diag}\left(\mathbf{C}_{\mathbf{z}_{r}}\right)^{-\frac{1}{2}}$ and the cross-correlation matrix between $\mathbf{z}_{\mathrm{R}_{r}}$ and $\mathbf{x}_{\mathrm{R}}$ is given by

$$
\mathbf{C}_{\mathbf{z}_{\mathrm{R}_{r}} \mathbf{x}_{\mathrm{R}}}=\mathbf{B} \hat{\mathbf{H}}_{\mathrm{R}} \mathbf{C}_{\mathbf{x}_{\mathrm{R}}}=\frac{1}{2} \mathbf{B} \hat{\mathbf{H}}_{\mathrm{R}}
$$

where due to the real-valued notation of the system $\mathbf{C}_{\mathbf{x}_{\mathrm{R}}}=E\left[\mathbf{x}_{\mathrm{R}} \mathbf{x}_{\mathrm{R}}^{T}\right]=\frac{1}{2} \mathbf{I}_{2 N_{t}}$. Derivation: See Appendix B.4.

Moreover, the auto-correlation of $\mathbf{z}_{\mathrm{R}_{r}}$ is given by

$$
\mathbf{C}_{\mathbf{z}_{r}}=\frac{1}{2} \mathbf{B} \hat{\mathbf{H}}_{\mathrm{R}} \hat{\mathbf{H}}_{\mathrm{R}}^{T} \mathbf{B}^{T}+\mathbf{B} \boldsymbol{\Gamma}_{\mathrm{R}} \mathbf{B}^{T}+\frac{\sigma_{n}^{2}}{2} \mathbf{B B}^{T} .
$$

Derivation: See Appendix B.5.

The term $\boldsymbol{\Gamma}_{\mathrm{R}}$ in the last equation is calculated as follows

$$
\begin{aligned}
\boldsymbol{\Gamma}_{\mathrm{R}} & =E\left[\tilde{\mathbf{X}}_{\mathrm{R}} E\left[\varepsilon_{\mathrm{R}} \varepsilon_{\mathrm{R}}^{T}\right] \tilde{\mathbf{X}}_{\mathrm{R}}^{T}\right] \\
& =E\left[\tilde{\mathbf{X}}_{\mathrm{R}}\left(\mathbf{R}_{\mathbf{h}_{\mathrm{R}}}-\mathbf{R}_{\mathbf{h}_{\mathrm{R}}} \hat{\boldsymbol{\Phi}}_{\mathrm{R}}^{T} \mathbf{C}_{\mathbf{z}_{\mathcal{Q}_{p}}}^{-1} \hat{\boldsymbol{\Phi}}_{\mathrm{R}} \mathbf{R}_{\mathbf{h}_{\mathrm{R}}}\right) \tilde{\mathbf{X}}_{\mathrm{R}}^{T}\right] \\
& =E\left[\tilde{\mathbf{X}}_{\mathrm{R}} \mathbf{R}_{\mathbf{h}_{\mathrm{R}}} \tilde{\mathbf{X}}_{\mathrm{R}}^{T}-\tilde{\mathbf{X}}_{\mathrm{R}} \mathbf{R}_{\mathbf{h}_{\mathrm{R}}} \hat{\boldsymbol{\Phi}}_{\mathrm{R}}^{T} \mathbf{C}_{\mathbf{z}_{\mathcal{Q}_{p}}}^{-1} \hat{\boldsymbol{\Phi}}_{\mathrm{R}} \mathbf{R}_{\mathbf{h}_{\mathrm{R}}} \tilde{\mathbf{X}}_{\mathrm{R}}^{T}\right],
\end{aligned}
$$

where $E\left[\varepsilon_{\mathrm{R}} \varepsilon_{\mathrm{R}}^{T}\right]=\mathbf{R}_{\mathbf{h}_{\mathrm{R}}}-\mathbf{R}_{\mathbf{h}_{\mathrm{R}}} \hat{\boldsymbol{\Phi}}_{\mathrm{R}}^{T} \mathbf{C}_{\mathbf{z}_{\mathcal{Q}_{p}}}^{-1} \hat{\boldsymbol{\Phi}}_{\mathrm{R}} \mathbf{R}_{\mathbf{h}_{\mathrm{R}}}$, similarly as previously calculated in (4-13). In the following it is considered that the modulation scheme is QPSK. Hence, the expected value of $\tilde{\mathbf{X}}_{\mathrm{R}}$ can be calculated as a uniform discrete distribution. In this context, equation (5-17) can be rewritten as 


$$
\begin{aligned}
\boldsymbol{\Gamma}_{\mathrm{R}} & =\sum_{i=1}^{4^{N_{t}}} P\left(\tilde{\mathbf{X}}_{\mathrm{R}, i}\right)\left(\tilde{\mathbf{X}}_{\mathrm{R}, i} \mathbf{R}_{\mathbf{h}_{\mathrm{R}}} \tilde{\mathbf{X}}_{\mathrm{R}, i}^{T}-\tilde{\mathbf{X}}_{\mathrm{R}, i} \mathbf{R}_{\mathbf{h}_{\mathrm{R}}} \hat{\boldsymbol{\Phi}}_{\mathrm{R}}^{T} \mathbf{C}_{\mathbf{z}_{\mathcal{Q}_{p}}}^{-1} \hat{\mathbf{\Phi}}_{\mathrm{R}} \mathbf{R}_{\mathbf{h}_{\mathrm{R}}} \tilde{\mathbf{X}}_{\mathrm{R}, i}^{T}\right) \\
& =\frac{1}{4^{N_{t}}} \sum_{i=1}^{4^{N t}}\left(\tilde{\mathbf{X}}_{\mathrm{R}, i} \mathbf{R}_{\mathbf{h}_{\mathrm{R}}} \tilde{\mathbf{X}}_{\mathrm{R}, i}^{T}-\tilde{\mathbf{X}}_{\mathrm{R}, i} \mathbf{R}_{\mathbf{h}_{\mathrm{R}}} \hat{\mathbf{\Phi}}_{\mathrm{R}}^{T} \mathbf{C}_{\mathbf{z}_{\mathcal{Q}_{p}}}^{-1} \hat{\mathbf{\Phi}}_{\mathrm{R}} \mathbf{R}_{\mathbf{h}_{\mathrm{R}}} \tilde{\mathbf{X}}_{\mathrm{R}, i}^{T}\right) .
\end{aligned}
$$

\section{3}

\section{Greedy Search Algorithm}

The proposed Greedy Search algorithm implies that in each search cycle, the comparator configuration with the highest MSE reduction is selected. In a nutshell, the proposed algorithm searches for each individual comparator sequentially over all possible combinations for the minimum MSE input combination. In this process, the comparators sequentially change their inputs according to the MSE criterion. The detailed process is summarized in Algorithm 1.

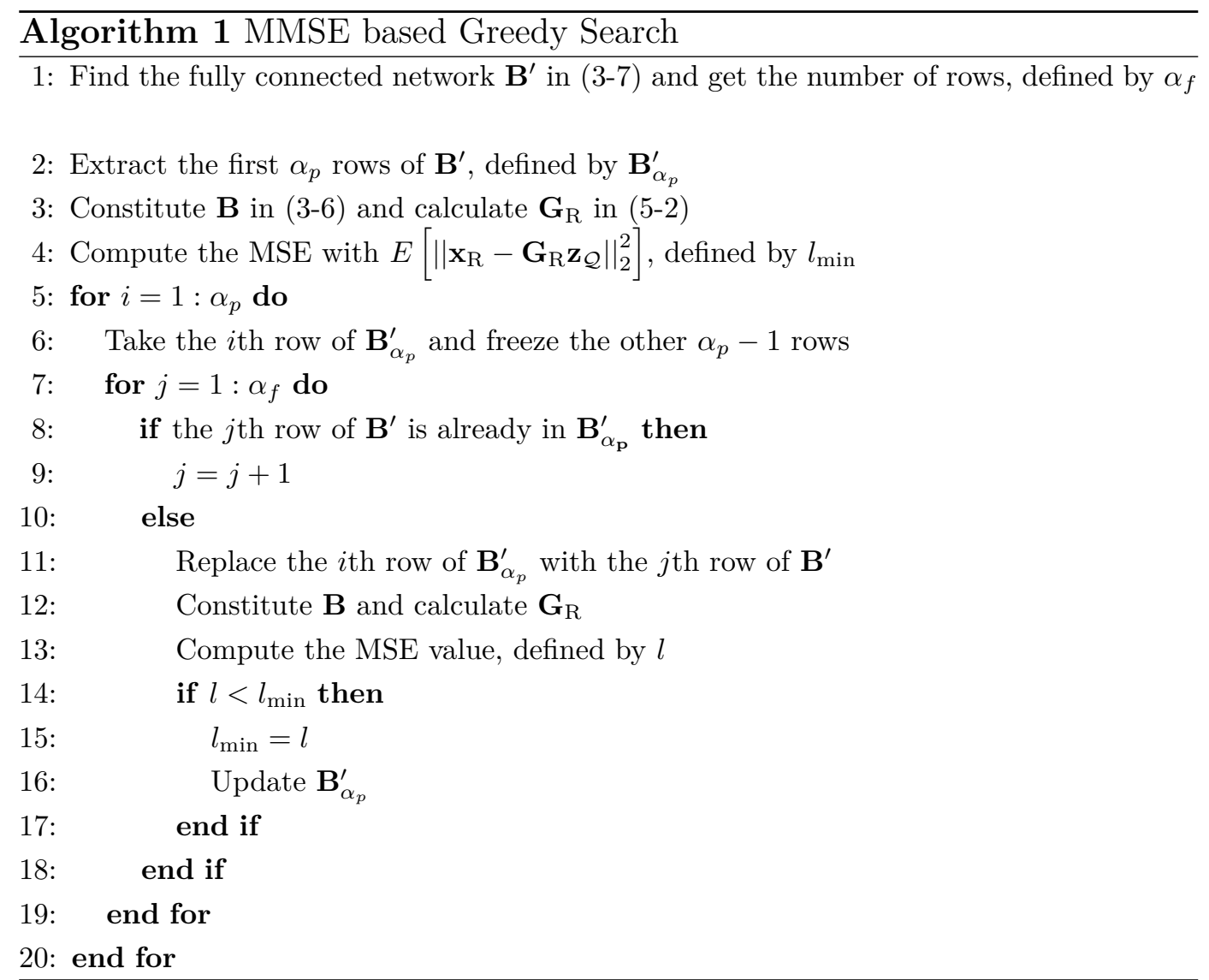

\section{4}

\section{Analysis}




\section{4 .1}

\section{Computational Complexity}

In this subsection the computational complexity of the presented approaches is examined. The corresponding complexity orders are summarized in Table 5.1, where $\mathcal{O}(\cdot)$ is the big O notation. Note that the complexity order can be calculated as functions of the numbers of receive and transmit antennas $N_{r}$ and $N_{t}$ and additional comparators $\alpha_{f}$ or $\alpha_{p}$.

Table 5.1: Computational Complexity

\begin{tabular}{|c|c|c|}
\hline Approach & Network Design & LRA-MMSE Detection \\
\hline \hline No Network & - & $\mathcal{O}\left(\left(2 N_{r}\right)^{3}+2 N_{t}\left(2 N_{r}\right)^{2}+4 N_{r} N_{t}\right)$ \\
\hline Full Connection & - & $\mathcal{O}\left(\left(2 N_{r}+\alpha_{f}\right)^{3}+2 N_{t}\left(2 N_{r}+\alpha_{f}\right)^{2}+\right.$ \\
& & $\left.2 N_{t}\left(2 N_{r}+\alpha_{f}\right)\right)$ \\
\hline MMSE based & $\mathcal{O}\left(\alpha_{p}\left(\alpha_{f}-\alpha_{p}\right)\left(2 N_{r}+\alpha_{p}\right)^{3}\right)$ & $\mathcal{O}\left(\left(2 N_{r}+\alpha_{p}\right)^{3}+2 N_{t}\left(2 N_{r}+\alpha_{p}\right)^{2}+\right.$ \\
Greedy Search & - & $\mathcal{O}\left(\left(2 N_{t}\left(2 N_{r}+\alpha_{p}\right)\right)\right.$ \\
\hline Random Selection & $\left.-\alpha_{p}\right)^{3}+2 N_{t}\left(2 N_{r}+\alpha_{p}\right)^{2}+$ \\
& - & $\left.2 N_{t}\left(2 N_{r}+\alpha_{p}\right)\right)$ \\
\hline
\end{tabular}

\section{4 .2}

\section{Computational and Hardware Costs}

The required computational and hardware costs in terms of additional comparators are approximately calculated in Table 5.2. The values presented for the computational cost (arithmetic operations) are calculated using Table 5.1 and considering the following parameters: $N_{r}=10, N_{t}=2, \alpha_{f}=190$ and $\alpha_{p}=20$. The hardware cost values are based on the number of additional comparators needed in each approach.

Table 5.2: Computational and Hardware Costs

\begin{tabular}{|c|c|c|}
\hline Approach & Computational Cost & Hardware Cost* \\
\hline \hline No Network & $\mathcal{O}(9680)$ & - \\
\hline Full Connection & $\mathcal{O}(9438240)$ & 190 \\
\hline MMSE based Greedy Search & $\mathcal{O}(217670560)$ & 20 \\
\hline Random Selection & $\mathcal{O}(70560)$ & 20 \\
\hline
\end{tabular}

*The presented hardware costs don't include the crossbar switch required to select which antennas will be used as inputs on each comparator.

\section{5}

\section{Numerical Results}

In this section, an uplink single-cell 1-bit MIMO system with comparator network, $N_{t}=2$ is considered. The modulation scheme is QPSK and the SNR is defined as $10 \log \left(\frac{1}{\sigma_{n}^{2}}\right)$, which is the average receive SNR per user per antenna. 


\subsection{1}

\section{Proof of Concept: More Antennas versus Comparator Network}

In this subsection, systems with different number of receive antennas $\left(N_{r}\right)$ and perfect CSI at the receiver are considered. While performing the signal detection, the LRA-LMMSE detector from Section 5.1 is applied in the system. The BER performance plots are obtained by taking the average over 1000 different channels and 1000 noise realizations per channel.

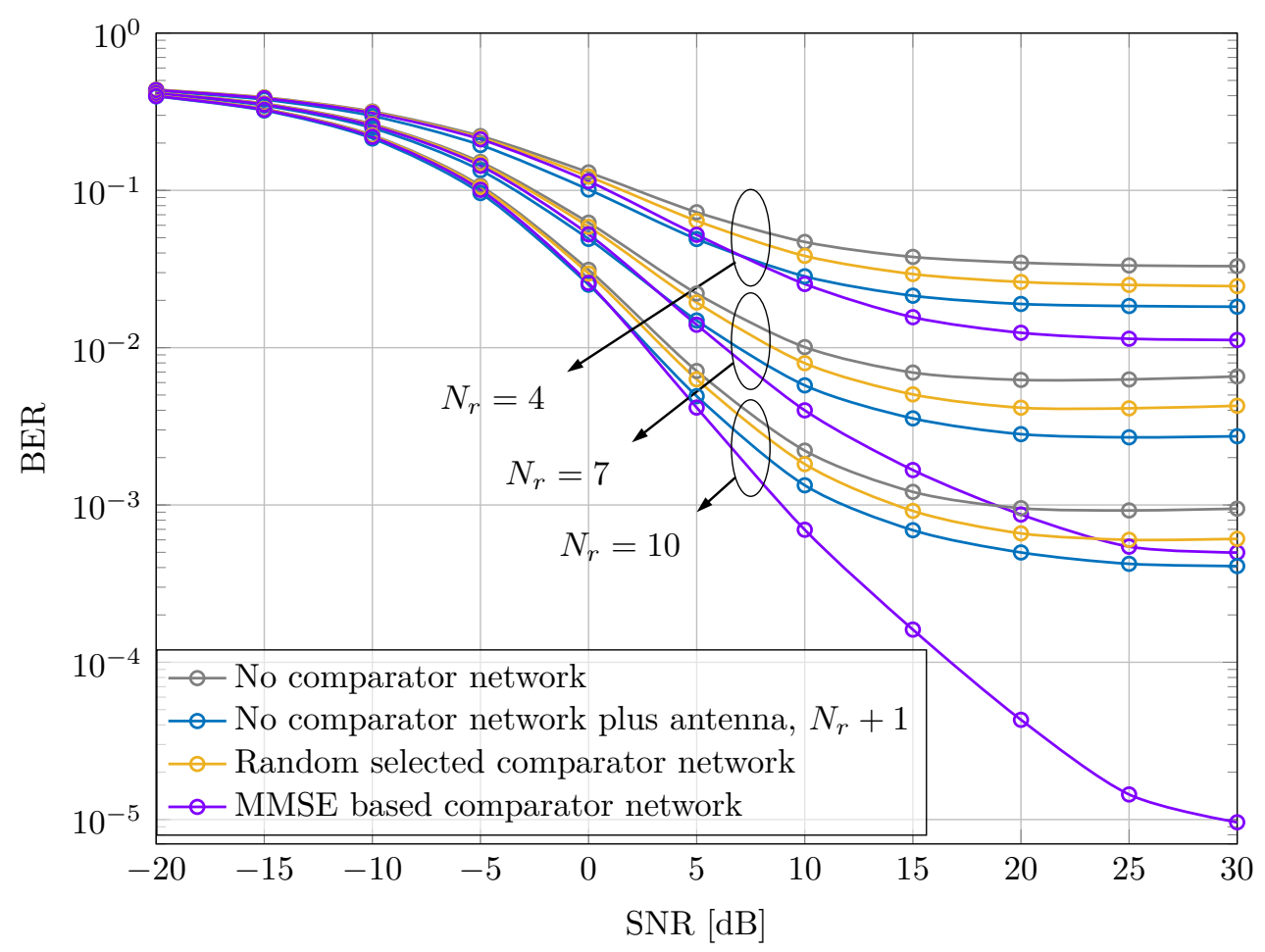

Figure 5.1: BER performance of LRA-LMMSE detectors in $2 \times N_{r}$ MIMO systems.

In order to verify the advantage of the addition of the comparator network, the performance of systems with partially connected networks are compared to systems with additional receive antennas. The configuration schemes with $N_{r}$ and $N_{r}+1$ receive antennas are considered, which means the addition of a single antenna element. Since each extra antenna would correspond to two comparators, to make a fair comparison, the total number of extra comparators utilized by the partially connected networks is always $\alpha_{p}=2$ in the presented simulations.

As we can see from Fig. 5.1, the random selected comparator network only has a benefit in comparison to the system without comparator network in a scenario with the same number of antennas. However, at high SNR the MMSE based Greedy Search outperforms the larger array because the extra virtual 
channels are selected with the lowest MSE approach, making the added virtual channels better than the extra physical channel for all different simulated $N_{r}$.

\section{5 .2}

\section{Proposed LRA-LMMSE Detector}

In this subsection, it is considered a system with $N_{r}=10$. For the signal detection, the LRA-LMMSE detector is applied in the system. The BER performance plots are obtained by taking the average over 2000 different channels and 2000 noise realizations per channel.

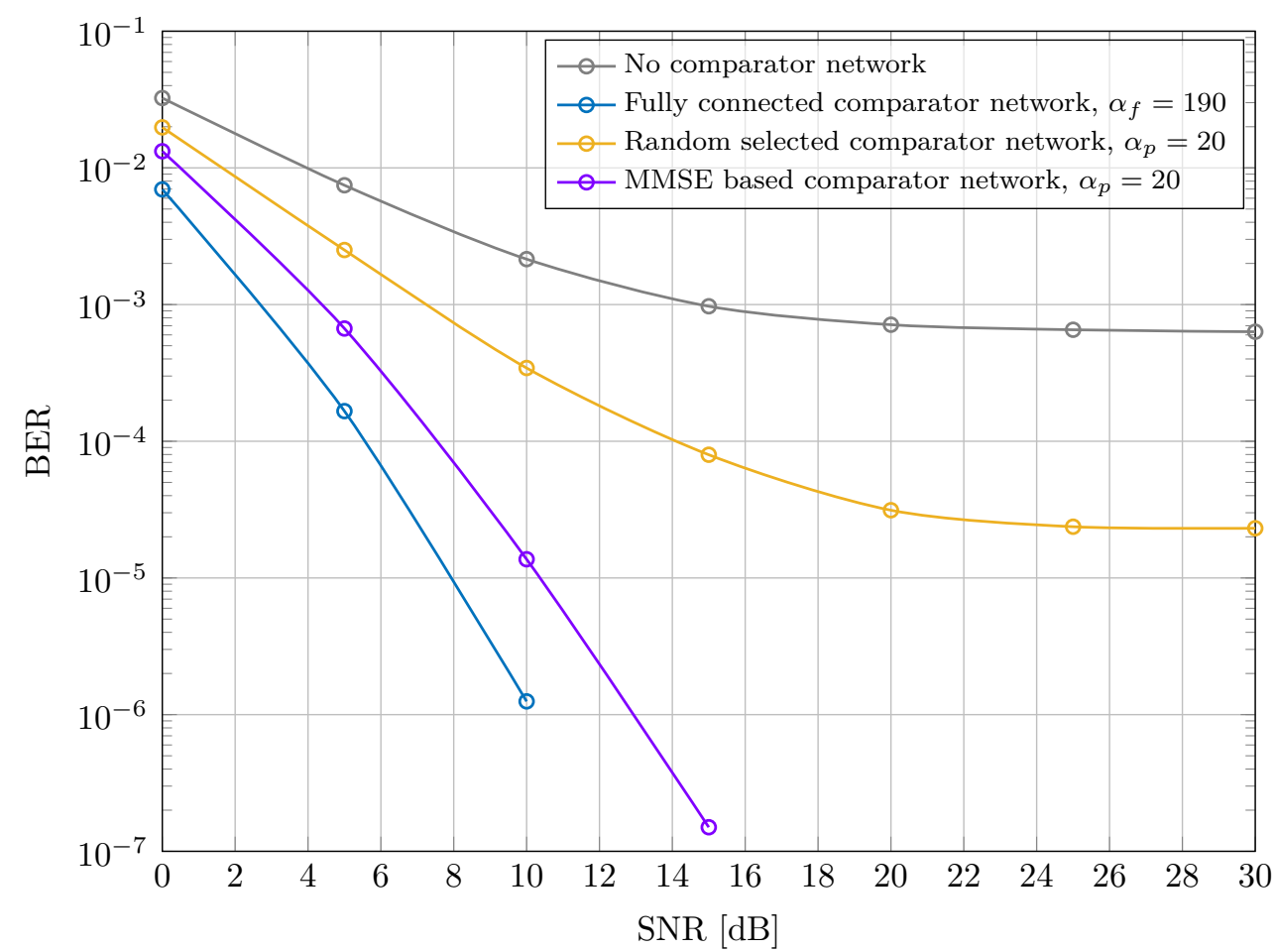

Figure 5.2: BER performance of LRA-LMMSE detectors in $2 \times 10$ MIMO systems.

The LRA-LMMSE detector BER performance of fully and partially connected networks under perfect CSI are shown in Fig. 5.2, where partially connected refers to comparator networks of, in this case, $\alpha_{p}=2 N_{r}=20$ comparators. The case of fully connected networks refers to comparator networks with, in this case, $\alpha_{f}=\left(\begin{array}{c}2 N_{r} \\ 2\end{array}\right)=190$ comparators.

Simulation results show that the system with fully connected network achieves the best BER performance with the cost of a large number of comparators and high computational complexity. In the partially connected networks, the MMSE based Greedy Search outperforms the random selection approach especially at high SNR, where the error floor is eliminated. A surprising observation is that the Greedy Search approach has almost the 
same BER performance as the fully connected method but with much less comparators. This shows great advantages of the greedy search based partially connected network. However, also the approach with the comparator network using random selected inputs is beneficial in terms of BER. While making comparison with the approach without additional comparator network, it can be seen that by adding extra 20 comparators the performance gain is significant and the error floor goes down largely.

However, it should be mentioned that although the greedy search approach yields comparable good BER performance with less required comparators, its computational complexity is the highest among all the approaches due to its sequential search for the least MSE values.

\subsection{3}

\section{Robust LRA-LMMSE Detector}

In this subsection, it is considered a system with $N_{r}=4$. The pilot sequences are column-wise orthogonal with length $\tau=N_{t}=2$, i.e., $\boldsymbol{\Phi}^{T} \boldsymbol{\Phi}=$ $\tau \mathbf{I}_{N_{t}}$. The BER performance plots are obtained by taking the average over 2000 different channels and 2000 noise realizations per channel.

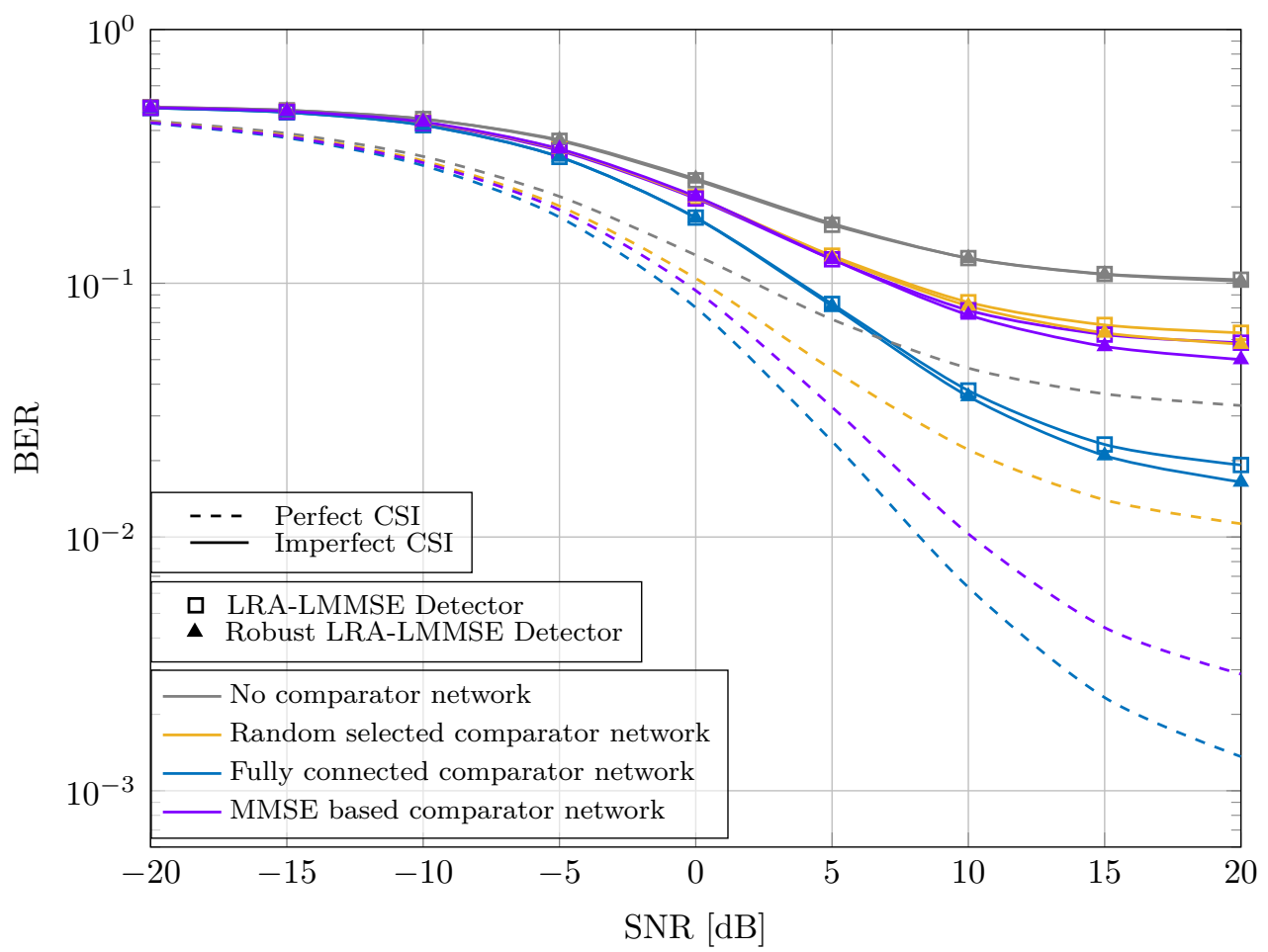

Figure 5.3: BER performance of robust LRA-LMMSE detectors in $2 \times 4$ MIMO systems.

The BER performance of the robust LRA-LMMSE detector with fully and partially connected networks is illustrated in Fig. 5.3. In these experiments, 


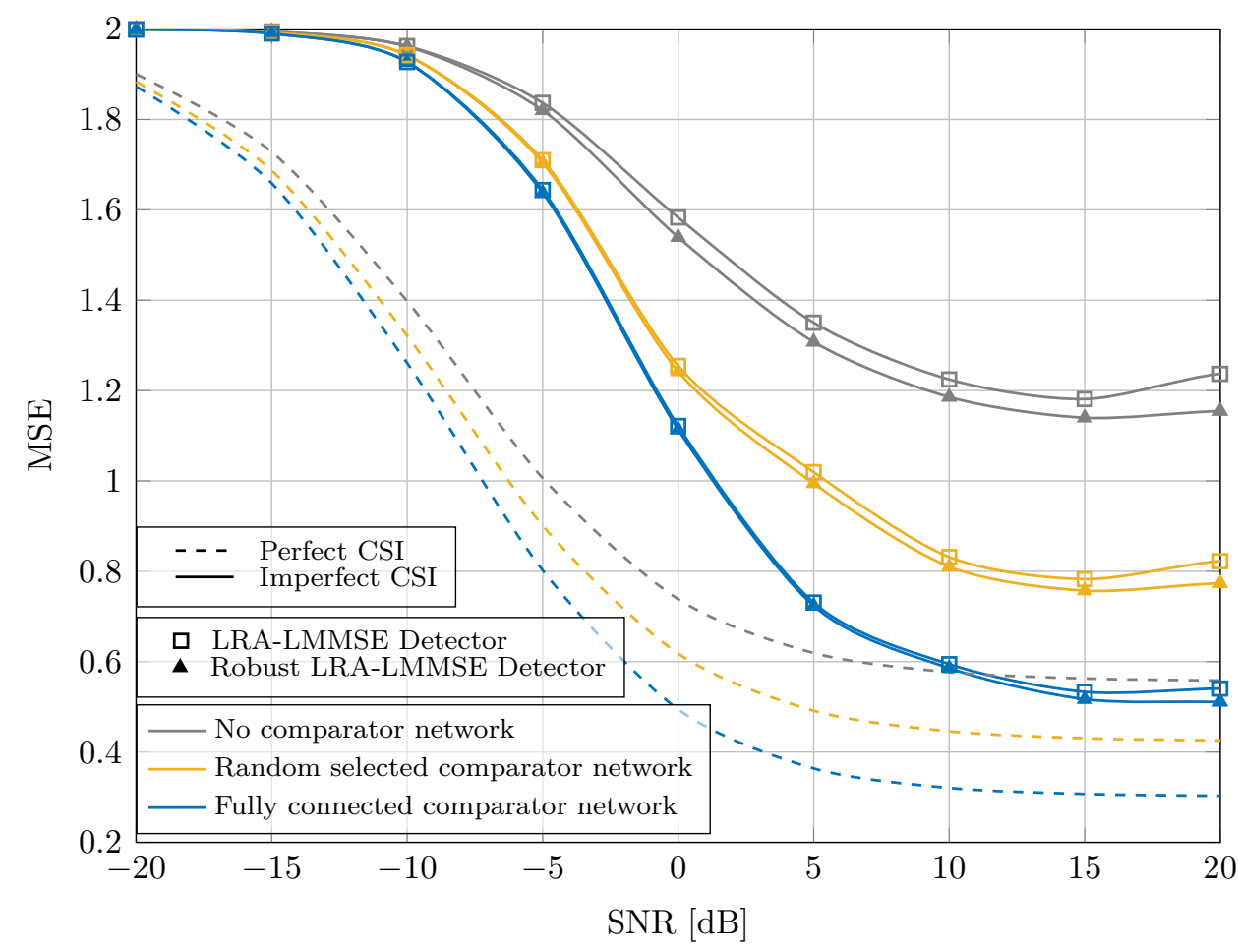

Figure 5.4: MSE comparisons of robust LRA-LMMSE detectors in $2 \times 4$ MIMO systems.

the partially connected networks have $\alpha_{p}=2 N_{r}=8$ comparators, while the fully connected have $\alpha_{f}=\left(\begin{array}{c}2 N_{r} \\ 2\end{array}\right)=28$ comparators. The LRA-LMMSE detector is based on the channel estimate while the robust LRA-LMMSE detector combines the proposed channel estimate with the corresponding estimation error statistics. As expected, simulation results show that the robust LRA-LMMSE detector obtains a moderate performance advantage in comparison to the non-robust detector. This benefit is not only in terms of BER but also MSE as shown in Fig. 5.4, which is the design objective of the detectors.

\section{6}

\section{Summary}

In this chapter, expressions for the proposed comparator network based LRA-LMMSE detectors with 1-bit quantization are derived. The Greedy Search algorithm based on the MSE selection criterion is presented. The additional comparator network provides additional information about the received signal which can be used to reduce the BER performance with only a slight increase in hardware cost and required computational complexity. Numerical results show that adding virtual channels by using a comparator network can be better than adding extra physical channels which corresponds 
to additional receive antennas in terms of BER. Moreover, simulation results show that the proposed partially connected networks, especially the MMSE based Greedy Search approach, require less comparators while introducing small performance degradation compared with the proposed fully connected networks. Furthermore, numerical results show that the proposed robust LRALMMSE detector have moderate performance advantage when compared to the non-robust one. 


\section{6}

\section{Sum Rate Analysis}

In this chapter, a data transmission stage is considered and a lower bound on the ergodic sum rate is build up for the LRA-LMMSE receiver by employing the proposed channel estimator from Chapter 4 and its corresponding estimation error. Then, the numerical results are demonstrating the potential of the proposed system.

\section{1}

\section{Data Transmission with LRA-LMMSE Receiver}

It is considered that in the data transmission stage the $N_{t}$ users simultaneously transmit their data symbols represented by the vector $\mathbf{x}_{R}$ to the BS, which is a stacked vector with real and imaginary parts. In the present study, real and imaginary parts represent independent data symbols. After processed by the comparators, the quantized signal can be expressed as

$$
\begin{aligned}
\mathbf{z}_{\mathcal{Q}_{d}} & =\mathcal{Q}\left(\mathbf{z}_{\mathrm{R}_{d}}\right)=\mathcal{Q}\left(\mathbf{B} \mathbf{y}_{\mathrm{R}_{d}}\right)=\mathcal{Q}\left(\mathbf{B} \mathbf{H}_{\mathrm{R}} \mathbf{x}_{\mathrm{R}}+\mathbf{B} \mathbf{n}_{\mathrm{R}_{d}}\right) \\
& =\mathbf{A}_{\mathrm{R}_{d}} \mathbf{B} \mathbf{H}_{\mathrm{R}} \mathbf{x}_{\mathrm{R}}+\mathbf{A}_{\mathrm{R}_{d}} \mathbf{B} \mathbf{n}_{\mathrm{R}_{d}}+\mathbf{n}_{\mathrm{R}_{q, d}},
\end{aligned}
$$

where the same definitions from Chapter 4 apply, but with the subscript $p$ replaced by $d$, since we changed from the pilots to the data transmission stage. Then, the LRA-LMMSE channel estimate (4-12) is used to compute a linear receiver which provides an estimate of the data symbols transmitted from the $N_{t}$ users. In this context, the quantized signal is separated into $2 N_{t}$ streams by multiplying the signal with the receiver filter matrix defined in (5-2) as $\mathbf{G}_{\mathrm{R}}=\mathbf{C}_{\mathbf{z}_{\mathcal{Q}_{d}}}^{-1} \mathbf{C}_{\mathbf{z}_{\mathcal{Q}_{d}} \mathbf{x}_{\mathrm{R}}}$, which in this case is computed based on the estimated channel. Thereby, we obtain

$$
\begin{aligned}
\hat{\mathbf{x}}_{\mathrm{R}} & =\mathbf{G}_{\mathrm{R}} \mathbf{z}_{\mathcal{Q}_{d}}=\mathbf{G}_{\mathrm{R}}\left(\mathbf{A}_{\mathrm{R}_{d}} \mathbf{B} \mathbf{H}_{\mathrm{R}} \mathbf{x}_{\mathrm{R}}+\mathbf{A}_{\mathrm{R}_{d}} \mathbf{B n}_{\mathrm{R}_{d}}+\mathbf{n}_{\mathrm{R}_{q, d}}\right) \\
& =\mathbf{G}_{\mathrm{R}} \mathbf{A}_{\mathrm{R}_{d}} \mathbf{B}\left(\hat{\mathbf{H}}_{\mathrm{R}} \mathbf{x}_{\mathrm{R}}+\mathcal{E}_{\mathrm{R}} \mathbf{x}_{\mathrm{R}}\right)+\mathbf{G}_{\mathrm{R}} \mathbf{A}_{\mathrm{R}_{d}} \mathbf{B n}_{\mathrm{R}_{d}}+\mathbf{G}_{\mathrm{R}} \mathbf{n}_{\mathrm{R}_{q, d}},
\end{aligned}
$$

where $\hat{\mathbf{H}}_{\mathrm{R}}$ is the estimated channel matrix described by (5-8) and $\mathcal{E}_{\mathrm{R}}=$ $\mathbf{H}_{\mathrm{R}}-\hat{\mathbf{H}}_{\mathrm{R}}$ is the channel estimation error matrix.

In the sum rate analysis it is considered that each user corresponds to two real-valued channels. Then, the $k$ th element represents an estimate of the signal of the $k$ th real-valued channel, similarly as in [16], with $k \in\left[1,2 N_{t}\right]$, 
which reads as

$$
\begin{aligned}
\hat{\mathbf{x}}_{\mathrm{R}_{k}}= & \underbrace{\mathbf{g}_{\mathrm{R}_{k}}^{T} \mathbf{A}_{\mathrm{R}_{d}} \mathbf{B} \hat{\mathbf{h}}_{\mathrm{R}_{k}} \mathbf{x}_{\mathrm{R}_{k}}}_{\text {desired signal }}+\underbrace{\mathbf{g}_{\mathrm{R}_{k}}^{T} \sum_{i \neq k}^{K} \mathbf{A}_{\mathrm{R}_{d}} \mathbf{B} \hat{\mathbf{h}}_{\mathrm{R}_{i}} \mathbf{x}_{\mathrm{R}_{i}}}_{\text {interference }}+\underbrace{\mathbf{g}_{\mathrm{R}_{k}}^{T} \sum_{i=1}^{K} \mathbf{A}_{\mathrm{R}_{d}} \mathbf{B} \varepsilon_{\mathrm{R}_{i}} \mathbf{x}_{\mathrm{R}_{i}}}_{\text {channel estimation error }} \\
& +\underbrace{\mathbf{g}_{\mathrm{R}_{k}}^{T} \mathbf{A}_{\mathrm{R}_{d}} \mathbf{B} \mathbf{n}_{\mathrm{R}_{d}}}_{\text {AWGN noise }}+\underbrace{\mathbf{g}_{\mathrm{R}_{k}}^{T} \mathbf{n}_{\mathrm{R}_{q, d}}}_{\text {quant. noise }},
\end{aligned}
$$

where $\mathbf{g}_{\mathrm{R}_{k}}^{T}$ is the $k$ th row of $\mathbf{G}_{\mathrm{R}}$ and $\hat{\mathbf{h}}_{\mathrm{R}_{k}}$ is the $k$ th column of $\hat{\mathbf{H}}_{\mathrm{R}}$. Moreover, $\varepsilon_{\mathrm{R}_{i}}$ is the $i$ th column of the matrix $\mathcal{E}_{\mathrm{R}}$.

\section{2}

\section{Lower Bounding the Sum Rate}

Since the Gaussian noise case corresponds to the worst case scenario, we can find a lower bound for the achievable rate by interpreting the quantization noise as Gaussian, with an equivalent noise covariance matrix [33]. In this regard, the equivalent noise covariance matrix is given by

$$
\mathbf{C}_{\mathbf{n}_{\mathrm{R}_{q, d}}}=\mathbf{C}_{\mathbf{z}_{\mathcal{Q}_{d}}}-\mathbf{A}_{\mathrm{R}_{d}} \mathbf{C}_{\mathbf{z}_{d}} \mathbf{A}_{\mathrm{R}_{d}}^{T},
$$

where $\mathbf{C}_{\mathbf{z}_{\mathcal{Q}_{d}}}=E\left[\mathbf{z}_{\mathcal{Q}_{d}} \mathbf{z}_{\mathcal{Q}_{d}}^{T}\right]$ is the auto-correlation matrix of the quantized data signal, like in (4-11), and $\mathbf{C}_{\mathbf{z}_{d}}=E\left[\mathbf{z}_{\mathrm{R}_{d}} \mathbf{z}_{\mathrm{R}_{d}}^{T}\right]=\frac{1}{2} \mathbf{B} \mathbf{H}_{\mathrm{R}} \mathbf{H}_{\mathrm{R}}^{T} \mathbf{B}^{T}+\frac{\sigma_{n}^{2}}{2} \mathbf{B B}^{T}$ is the auto-correlation matrix of the received data signal, as calculated in (5-6).

Derivation: See Appendix C.1

Using this approach and by considering Gaussian signaling, the ergodic achievable rate per real-valued channel is lower bounded by

$$
I_{\mathrm{R}_{k}}=E\left[\frac{1}{2} \log _{2}\left(1+\frac{\left|\mathbf{d}_{\mathrm{R}_{k}} \hat{\mathbf{h}}_{\mathrm{R}_{k}}\right|^{2}}{\sum_{i \neq k}^{K}\left|\mathbf{d}_{\mathrm{R}_{k}} \hat{\mathbf{h}}_{\mathrm{R}_{i}}\right|^{2}+\sum_{i=1}^{K}\left|\mathbf{d}_{\mathrm{R}_{k}} \varepsilon_{\mathrm{R}_{i}}\right|^{2}+\left.\sigma_{n}^{2}|| \mathbf{d}_{\mathrm{R}_{k}}\right|_{2} ^{2}+2 \mathbf{g}_{\mathrm{R}_{k}}^{T} \mathbf{C}_{\mathbf{n}_{\mathrm{R}_{q, d}} \mathbf{g}_{\mathrm{R}_{k}}}}\right)\right],
$$

where $\mathbf{d}_{\mathrm{R}_{k}}=\mathbf{g}_{\mathrm{R}_{k}}^{T} \mathbf{A}_{\mathrm{R}_{d}} \mathbf{B}$ and the expectation operator is taken with respect to channel realizations and channel estimation realizations.

According to prior literature [33], this method provides an accurate lower bound especially for the low SNR regime. Finally, the sum rate is lowerbounded by $\sum_{k=1}^{K} I_{\mathrm{R}_{k}}$.

Derivation: See Appendix C.2

\section{3}

\section{Numerical Results}

In this section, an uplink single-cell 1-bit MIMO system with comparator network, $N_{t}=2$ and $N_{r}=4$ is considered. The pilot sequences are column-wise orthogonal with length $\tau=N_{t}=2$, i.e., $\boldsymbol{\Phi}^{T} \boldsymbol{\Phi}=\tau \mathbf{I}_{N_{t}}$. The SNR is defined as 
$10 \log \left(\frac{1}{\sigma_{n}^{2}}\right)$, which is the average receive SNR per user per antenna. The sum rate plots are obtained by taking the average over 2000 different channels and 2000 noise realizations per channel.

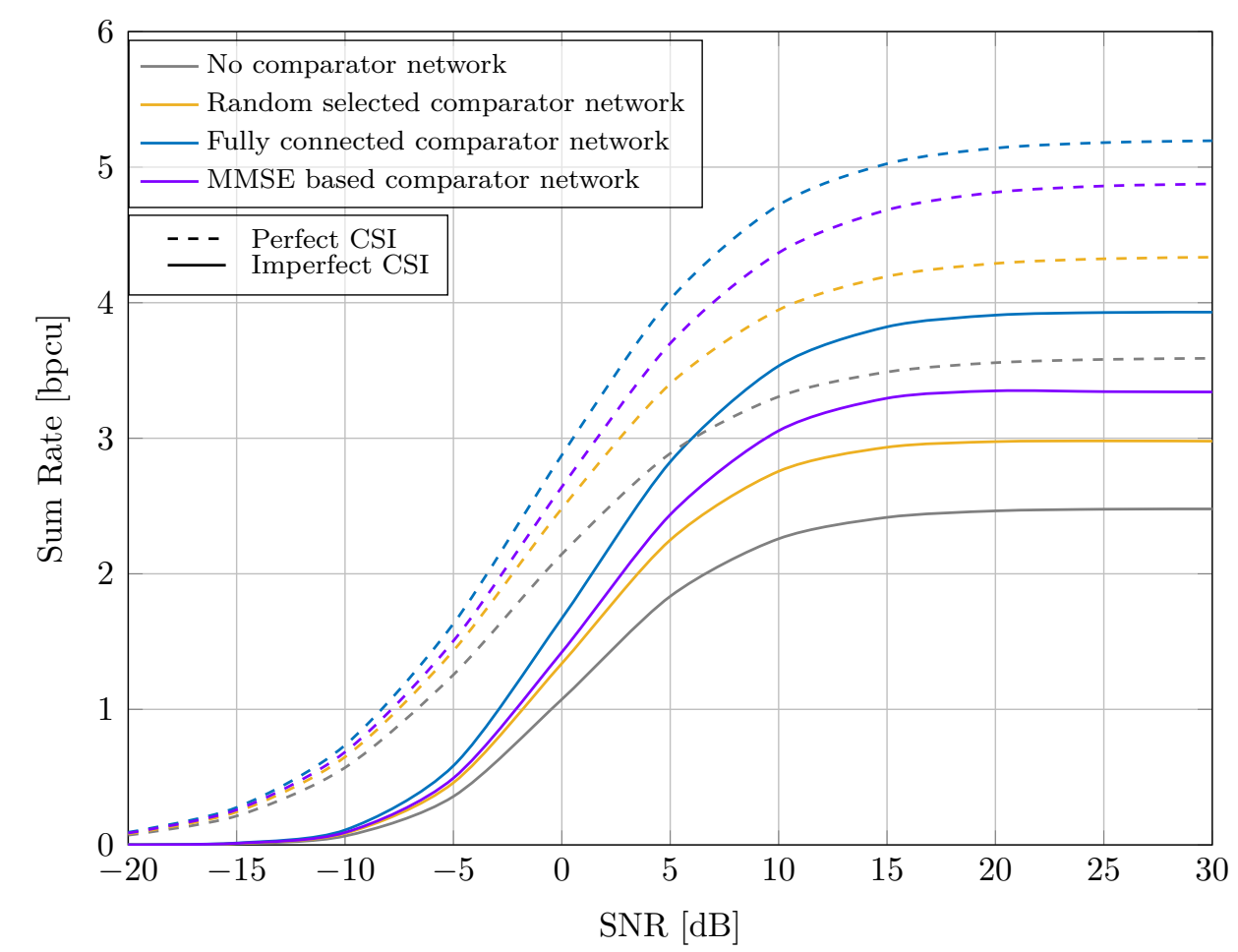

Figure 6.1: Sum Rate comparisons of LRA-LMMSE channel estimators in $2 \times 4$ MIMO systems.

The comparison between the lower bound of the ergodic sum rate with and without the comparator network is presented. The sum rate versus SNR for the systems under perfect and imperfect CSI is shown in Fig. 6.1, which indicates a significant benefit for the system that utilizes the additional comparator networks. In this experiment, the partially connected networks have $\alpha_{p}=2 N_{r}=8$ comparators, while the fully connected have $\alpha_{f}=\left(\begin{array}{c}2 N_{r} \\ 2\end{array}\right)=$ 28 comparators. We can see that the system with fully connected network achieves the best sum rate performance, followed by the MMSE based Greedy Search and the random selected inputs. Note that the increased sum rate is not only due to the comparator network aided receive processing but also due to a more accurate channel estimation.

\section{4}

\section{Summary}

This chapter has shown that by considering that the BS employs the linear receiver derived in Chapter 5 and using the proposed channel estimate and the corresponding estimation error from Chapter 4, we have derived 
an expression for lower bounding the ergodic sum rate. Simulation results show that the proposed comparator network based system outperforms the conventional 1-bit receiver in terms of sum rates. 


\section{7}

\section{Conclusion and Future Work}

In this thesis it is proposed a novel MIMO receiver architecture with the use of coarse quantization ADCs. Different from conventional systems, the proposed MIMO receiver includes a comparator network with binary outputs which can compare signals from different antennas. The resulting extension can be interpreted as a number of additional virtual channels. The additional virtual channels of the proposed low-resolution MIMO receiver contain additional information which aid the detection and channel estimation processes with only a slight increase in hardware cost and required computational complexity. In this context, channel estimation, signal detection and sum rate schemes are developed for the proposed system.

Two types of comparator networks are proposed, fully and partially connected networks. Simulation results show that the proposed partially connected networks, especially the MMSE based greedy search approach, require less comparators while introducing small performance degradation compared with the proposed fully connected networks.

Based on the proposed channel estimation and decoding algorithms, simulation results show that the proposed MIMO receiver architectures are superior to the conventional 1-bit quantization receiver methods in terms of BER and MSE. Moreover, numerical results show that adding virtual channels by using comparator network can be better than adding extra physical channels which corresponds to additional receive antennas in terms of BER. Furthermore, the robust LRA-LMMSE detector has been developed which is based on the channel estimation statistics. Simulation results indicate a moderate advantage in performance in terms of BER and MSE in comparison to the non-robust detector. Also, by considering that the base station employs a linear receiver and using the proposed channel estimate and the corresponding estimation error, it has been derived an expression for lower bounding the ergodic sum rate. Numerical results show that the corresponding sum rate increases significantly when adding a comparator network to the system.

Several future research topic are suggested:

- Investigate more sophisticated channel estimation techniques in conjunction with the comparator network. 
- Investigate the proposed system model in conjunction with spatial or temporal oversampling.

- Development of efficient search algorithm: design with a lower computational cost and investigation of implementing neural networks. The main goal would be trying to approach the fully connected network performance.

- Include a coding scheme in order to have a more practical setting. For example, an iterative detection and decoding scheme for the comparator network.

- Consideration of imperfect 1-bit quantization with random threshold mismatch.

- Computation of theoretical limits for the channel estimation such as Cramér-Rao lower bound. 


\section{Bibliography}

[1] LARSSON, E. G.; EDFORS, O.; TUFVESSON, F. ; MARZETTA, T. L.. Massive MIMO for next generation wireless systems. IEEE Commun. Mag., 52(2):186-195, Feb. 2014.

[2] WALDEN, R. H.. Analog-to-digital converter survey and analysis. IEEE J. Sel. Areas Commun., 17(4):539-550, Apr. 1999.

[3] JACOBSSON, S.; DURISI, G.; COLDREY, M.; GUSTAVSSON, U. ; STUDER, C.. Throughput analysis of massive MIMO uplink with low-resolution ADCs. IEEE Trans. Wireless Commun., 16(6):4038-4051, Jun. 2017.

[4] ZHANG, J.; DAI, L.; LI, X.; LIU, Y. ; HANZO, L.. On Low-Resolution ADCs in Practical 5G Millimeter-wave Massive MIMO Systems. IEEE Commun. Mag., 56(7):205-211, Jul. 2018.

[5] LIU, T.; TONG, J.; GUO, Q.; XI, J.; YU, Y. ; XIAO, Z.. Energy Efficiency of Massive MIMO Systems With Low-Resolution ADCs and Successive Interference Cancellation. IEEE Trans. Wireless Commun., 18(8):3987-4002, Aug. 2019.

[6] LANDAU, L. T. N.; DÖRPINGHAUS, M. ; FETTWEIS, G. P.. 1-Bit Quantization and Oversampling at the Receiver: Communication Over Bandlimited Channels With Noise. IEEE Communications Letters, 21(5):1007-1010, 2017.

[7] ÜÇÜNCÜ, A. B.; BJÖRNSON, E.; JOHANSSON, H.; YILMAZ, A. O. ; LARSSON, E. G.. Performance Analysis of Quantized Uplink Massive MIMO-OFDM With Oversampling Under Adjacent Channel Interference. IEEE Trans. Commun., p. 1-1, 2019.

[8] SHAO, Z.; LANDAU, L. T. N. ; DE LAMARE, R. C.. Sliding Window Based Linear Signal Detection Using 1-Bit Quantization and Oversampling for Large-Scale Multiple-Antenna Systems. In: 2018 IEEE STATISTICAL SIGNAL PROCESSING WORKSHOP (SSP), p. 183-187, Freiburg, Germany, Jun. 2018. 
[9] SHAO, Z.; LANDAU, L. T. N. ; DE LAMARE, R. C.. Channel Estimation for Large-Scale Multiple-Antenna Systems Using 1-Bit ADCs and Oversampling. IEEE Access, 8:85243-85256, 2020.

[10] SHAO, Z.; LANDAU, L. T. N. ; DE LAMARE, R. C.. Dynamic Oversampling for 1-Bit ADCs in Large-Scale Multiple-Antenna Systems. IEEE Transactions on Communications, 2021.

[11] RISI, C.; PERSSON, D. ; LARSSON, E. G.. Massive MIMO with 1-bit ADC. arXiv preprint arXiv:1404.7736, 2014.

[12] SHAO, Z.; LANDAU, L. ; D. LAMARE, R.. Adaptive RLS Channel Estimation and SIC for Large-Scale Antenna Systems with 1Bit ADCs. In: WSA 2018; 22ND INTERNATIONAL ITG WORKSHOP ON SMART ANTENNAS, Bochum, Germany, Mar. 2018.

[13] MO, J.; SCHNITER, P. ; HEATH, R. W.. Channel Estimation in Broadband Millimeter Wave MIMO Systems With Few-Bit ADCs. IEEE Trans. Signal Process., 66(5):1141-1154, Mar. 2018.

[14] XIONG, Y.; WEI, N. ; ZHANG, Z.. A Low-Complexity Iterative GAMP-Based Detection for Massive MIMO with LowResolution ADCs. In: 2017 IEEE WIRELESS COMMUNICATIONS AND NETWORKING CONFERENCE (WCNC), p. 1-6, 2017.

[15] CHOI, J.; MO, J. ; HEATH, R. W.. Near Maximum-Likelihood Detector and Channel Estimator for Uplink Multiuser Massive MIMO Systems with One-Bit ADCs. IEEE Transactions on Communications, 64(5):2005-2018, 2016.

[16] LI, Y.; TAO, C.; SECO-GRANADOS, G.; MEZGHANI, A.; SWINDLEHURST, A. L. ; LIU, L.. Channel Estimation and Performance Analysis of One-Bit Massive MIMO Systems. IEEE Transactions on Signal Processing, 65(15):4075-4089, Aug. 2017.

[17] STEIN, M. S.; BAR, S.; NOSSEK, J. A. ; TABRIKIAN, J.. Performance Analysis for Channel Estimation With 1-Bit ADC and Unknown Quantization Threshold. IEEE Trans. Signal Process., 66(10):25572571, May 2018.

[18] KIM, I.; CHOI, J.. Channel estimation via gradient pursuit for mmWave massive MIMO systems with one-bit ADCs. EURASIP Journal on Wireless Communications and Networking, 2019(1):289, 2019. 
[19] KIM, H.; CHOI, J.. Channel estimation for spatially/temporally correlated massive MIMO systems with one-bit ADCs. EURASIP Journal on Wireless Communications and Networking, 2019(1):267, 2019.

[20] MOLLÉN, C.; CHOI, J.; LARSSON, E. G. ; HEATH, R. W.. Uplink Performance of Wideband Massive MIMO With One-Bit ADCs. IEEE Transactions on Wireless Communications, 16(1):87-100, 2017.

[21] SHAO, Z.; DE LAMARE, R. C. ; LANDAU, L. T. N.. Iterative Detection and Decoding for Large-Scale Multiple-Antenna Systems With 1-Bit ADCs. IEEE Wireless Commun. Lett., 7(3):476-479, Jun. 2018.

[22] JEON, Y.; LEE, N.; HONG, S. ; HEATH, R. W.. One-Bit Sphere Decoding for Uplink Massive MIMO Systems With One-Bit ADCs. IEEE Trans. Wireless Commun., 17(7):4509-4521, Jul. 2018.

[23] B. CUNhA, T. E.; DE LAMARE, R. C.; FERREIRA, T. N. ; HÄLSIG, T.. Joint Automatic Gain Control and MMSE Receiver Design for Quantized Multiuser MIMO Systems. In: 2018 15TH INTERNATIONAL SYMPOSIUM ON WIRELESS COMMUNICATION SYSTEMS (ISWCS), p. 1-5, 2018.

[24] PIRZADEH, H.; SECO-GRANADOS, G.; RAO, S. ; SWINDLEHURST, A. L.. Spectral Efficiency of One-Bit Sigma-Delta Massive MIMO. IEEE Journal on Selected Areas in Communications, 38(9):2215-2226, 2020.

[25] RAO, S.; SWINDlEhURST, A. L. ; PIRZADEH, H.. Massive MIMO Channel Estimation with 1-Bit Spatial Sigma-delta ADCs. In: ICASSP 2019 - 2019 IEEE INTERNATIONAL CONFERENCE ON ACOUSTICS, SPEECH AND SIGNAL PROCESSING (ICASSP), p. 4484-4488, 2019.

[26] USMAN, O. B.; JEDDA, H.; MEZGHANI, A. ; NOSSEK, J. A.. MMSE precoder for massive MIMO using 1-bit quantization. In: 2016 IEEE INTERNATIONAL CONFERENCE ON ACOUSTICS, SPEECH AND SIGNAL PROCESSING (ICASSP), p. 3381-3385, 2016.

[27] LANDAU, L. T. N.; DE LAMARE, R. C.. Branch-and-Bound Precoding for Multiuser MIMO Systems With 1-bit Quantization. IEEE Wireless Communications Letters, 6(6):770-773, 2017.

[28] MELO, D. M. V.; LANDAU, L. T. N. ; DE LAMARE, R. C.. Zero-Crossing Precoding with MMSE Criterion for Channels with 1-BIT Quantization and Oversampling. In: WSA 2020; 24TH INTERNATIONAL ITG WORKSHOP ON SMART ANTENNAS, p. 1-6, 2020. 
[29] LOPES, E. S. P.; LANDAU, L. T. N.. Optimal Precoding for Multiuser MIMO Systems With Phase Quantization and PSK Modulation via Branch-and-Bound. IEEE Wireless Communications Letters, 9(9):1393-1397, 2020.

[30] FeRnAndeS, A. B. L. B.; LANDAU, L. T. N.. Comparator network aided channel estimation and achievable rates for MIMO receivers with 1-bit quantization. In: 2021 IEEE STATISTICAL SIGNAL PROCESSING WORKSHOP (SSP), p. 381-385, 2021.

[31] FERNANDES, A. B. L. B.; SHAO, Z.; LANDAU, L. T. N. ; DE LAMARE, R. C.. Comparator network aided detection for MIMO receivers with 1-bit quantization. In: 2020 54TH ASILOMAR CONFERENCE ON SIGNALS, SYSTEMS, AND COMPUTERS, p. 384-387, 2020.

[32] COLERI, S.; ERGEN, M.; PURI, A. ; BAHAI, A.. Channel estimation techniques based on pilot arrangement in OFDM systems. IEEE Transactions on Broadcasting, 48(3):223-229, 2002.

[33] MEZGHANI, A.; NOSSEK, J.. Capacity Lower Bound of MIMO Channels with Output Quantization and Correlated Noise. In: 2012 INTERNATIONAL SYMPOSIUM ON INFORMATION THEORY, Cambridge, MA, Jul. 2012.

[34] JACOVITTI, G.; NERI, A.. Estimation of the autocorrelation function of complex Gaussian stationary processes by amplitude clipped signals. IEEE Trans. Inf. Theory, 40(1):239-245, Jan. 1994.

[35] YANG, S.; HANZO, L.. Fifty Years of MIMO Detection: The Road to Large-Scale MIMOs. IEEE Communications Surveys Tutorials, 17(4):1941-1988, 2015.

[36] BUSSGANG, J. J.. Crosscorrelation functions of amplitudedistorted Gaussian signals. Res. Lab. Elec., MIT, Tech. Rep. 216, Mar. 1952.

[37] MEZGHANI, A.; NOSSEK, J. A.. Capacity lower bound of MIMO channels with output quantization and correlated noise. In: PROC. IEEE INT. SYMP. INFORM. THEORY (ISIT), p. 1732-1736, Cambridge, MA, USA, July 2012. 
A

\section{Linear Channel Estimation Derivations}

\section{A.1}

\section{Derivation of the Auto-Correlation Matrix of the Received Pilot Signal}

The auto-correlation matrix of $\mathbf{z}_{\mathrm{R}_{p}}$ from (4-9) is calculated below. By inserting (4-4) into the expectation operator, we get

$$
\begin{aligned}
& \mathbf{C}_{\mathbf{z}_{\mathrm{R}_{p}}}=E\left[\mathbf{z}_{\mathrm{R}_{p}} \mathbf{z}_{\mathrm{R}_{p}}^{T}\right]=E\left[\left(\mathbf{B}_{\text {eff }} \mathbf{y}_{\mathrm{R}_{p}}\right)\left(\mathbf{B}_{\mathrm{efff}} \mathbf{y}_{\mathrm{R}_{p}}\right)^{T}\right] \\
& =E\left[\left(\mathbf{B}_{\mathrm{eff}}\left(\tilde{\boldsymbol{\Phi}}_{\mathrm{R}} \mathbf{h}_{\mathrm{R}}+\mathbf{n}_{\mathrm{R}_{p}}\right)\right)\left(\left(\tilde{\mathbf{\Phi}}_{\mathrm{R}} \mathbf{h}_{\mathrm{R}}+\mathbf{n}_{\mathrm{R}_{p}}\right)^{T} \mathbf{B}_{\text {eff }}^{T}\right)\right] \\
& =E\left[\left(\mathbf{B}_{\mathrm{eff}}\left(\tilde{\boldsymbol{\Phi}}_{\mathrm{R}} \mathbf{h}_{\mathrm{R}}+\mathbf{n}_{\mathrm{R}_{p}}\right)\right)\left(\left(\mathbf{h}_{\mathrm{R}}^{T} \tilde{\boldsymbol{\Phi}}_{\mathrm{R}}^{T}+\mathbf{n}_{\mathrm{R}_{p}}^{T}\right) \mathbf{B}_{\mathrm{eff}}^{T}\right)\right] \\
& =E\left[\left(\mathbf{B}_{\text {eff }} \tilde{\boldsymbol{\Phi}}_{\mathrm{R}} \mathbf{h}_{\mathrm{R}}+\mathbf{B}_{\text {eff }} \mathbf{n}_{\mathrm{R}_{p}}\right)\left(\mathbf{h}_{\mathrm{R}}^{T} \tilde{\boldsymbol{\Phi}}_{\mathrm{R}}^{T} \mathbf{B}_{\text {eff }}^{T}+\mathbf{n}_{\mathrm{R}_{p}}^{T} \mathbf{B}_{\text {eff }}^{T}\right)\right] \\
& =E\left[\mathbf{B}_{\mathrm{eff}} \tilde{\boldsymbol{\Phi}}_{\mathrm{R}} \mathbf{h}_{\mathrm{R}} \mathbf{h}_{\mathrm{R}}^{T} \tilde{\boldsymbol{\Phi}}_{\mathrm{R}}^{T} \mathbf{B}_{\mathrm{eff}}^{T}+\mathbf{B}_{\mathrm{eff}} \tilde{\boldsymbol{\Phi}}_{\mathrm{R}} \mathbf{h}_{\mathrm{R}} \mathbf{n}_{\mathrm{R}_{p}}^{T} \mathbf{B}_{\mathrm{eff}}^{T}\right. \\
& \left.+\mathbf{B}_{\text {eff }} \mathbf{n}_{\mathrm{R}_{p}} \mathbf{h}_{\mathrm{R}}^{T} \tilde{\boldsymbol{\Phi}}_{\mathrm{R}}^{T} \mathbf{B}_{\text {eff }}^{T}+\mathbf{B}_{\text {eff }} \mathbf{n}_{\mathrm{R}_{p}} \mathbf{n}_{\mathrm{R}_{p}}^{T} \mathbf{B}_{\text {eff }}^{T}\right] \\
& =\mathbf{B}_{\mathrm{eff}} \tilde{\boldsymbol{\Phi}}_{\mathrm{R}} \mathbf{R}_{\mathbf{h}_{\mathrm{R}}} \tilde{\boldsymbol{\Phi}}_{\mathrm{R}}^{T} \mathbf{B}_{\mathrm{eff}}^{T}+\mathbf{B}_{\mathrm{eff}} \mathbf{C}_{\mathbf{n}_{\mathrm{R}_{\mathbf{p}}}} \mathbf{B}_{\mathrm{eff}}^{T} \text {, }
\end{aligned}
$$

where it is considered that $\mathbf{h}_{\mathrm{R}}$ is uncorrelated with $\mathbf{n}_{\mathrm{R}_{p}}$. Thus, $E\left[\mathbf{h}_{\mathrm{R}} \mathbf{n}_{\mathrm{R}_{p}}^{T}\right]=$ $E\left[\mathbf{n}_{\mathrm{R}_{p}} \mathbf{h}_{\mathrm{R}}^{T}\right]=\mathbf{0}$.

\section{A. 2}

\section{Derivation of the LRA-LMMSE Optimal Filter and Channel Estimator}

Recalling the optimization problem from (4-10)

$$
\begin{aligned}
& \mathbf{W}_{\mathrm{R}, \text { LRA-LMMSE }}=\arg \min _{\mathbf{W}} E\left[\left\|\mathbf{h}_{\mathrm{R}}-\mathbf{W}_{\mathcal{Q}_{p}}\right\|_{2}^{2}\right] \\
& =E\left[\operatorname{tr}\left(\left(\mathbf{h}_{\mathrm{R}}-\mathbf{W} \mathbf{z}_{\mathcal{Q}_{p}}\right)\left(\mathbf{h}_{\mathrm{R}}-\mathbf{W z}_{\mathcal{Q}_{p}}\right)^{T}\right)\right] \\
& =E\left[\operatorname{tr}\left(\left(\mathbf{h}_{\mathrm{R}}-\mathbf{W z}_{\mathcal{Q}_{p}}\right)\left(\mathbf{h}_{\mathrm{R}}^{T}-\mathbf{z}_{\mathcal{Q}_{p}}^{T} \mathbf{W}^{T}\right)\right)\right] \\
& =E\left[\operatorname{tr}\left(\mathbf{h}_{\mathrm{R}} \mathbf{h}_{\mathrm{R}}^{T}-\mathbf{h}_{\mathrm{R}} \mathbf{z}_{\mathcal{Q}_{p}}^{T} \mathbf{W}^{T}-\mathbf{W} \mathbf{z}_{\mathcal{Q}_{p}} \mathbf{h}_{\mathrm{R}}^{T}+\mathbf{W} \mathbf{z}_{\mathcal{Q}_{p}} \mathbf{z}_{\mathcal{Q}_{p}}^{T} \mathbf{W}^{T}\right)\right] \\
& =E\left[\operatorname{tr}\left(\mathbf{h}_{\mathrm{R}} \mathbf{h}_{\mathrm{R}}^{T}-2 \mathbf{h}_{\mathrm{R}} \mathbf{z}_{\mathcal{Q}_{p}}^{T} \mathbf{W}^{T}+\mathbf{W} \mathbf{z}_{\mathcal{Q}_{p}} \mathbf{z}_{\mathcal{Q}_{p}}^{T} \mathbf{W}^{T}\right)\right] \\
& =\operatorname{tr}\left(\mathbf{R}_{\mathbf{h}_{\mathrm{R}}}\right)-2 \operatorname{tr}\left(E\left[\mathbf{h}_{\mathrm{R}} \mathbf{z}_{\mathcal{Q}_{p}}^{T} \mathbf{W}^{T}\right]\right)+\operatorname{tr}\left(E\left[\mathbf{W z}_{\mathcal{Q}_{p}} \mathbf{z}_{\mathcal{Q}_{p}}^{T} \mathbf{W}^{T}\right]\right) \text {. }
\end{aligned}
$$

Taking the partial derivative with respect to $\mathbf{W}^{T}$, we obtain 


$$
\frac{\partial E\left[\left\|\mathbf{h}_{\mathrm{R}}-\mathbf{W} \mathbf{z}_{\mathcal{Q}_{p}}\right\|_{2}^{2}\right]}{\partial \mathbf{W}^{T}}=-E\left[\mathbf{h}_{\mathrm{R}} \mathbf{z}_{\mathcal{Q}_{p}}^{T}\right]+\mathbf{W} E\left[\mathbf{z}_{\mathcal{Q}_{p}} \mathbf{z}_{\mathcal{Q}_{p}}^{T}\right] .
$$

Equaling (A-3) to zero and inserting (4-6), the LRA-LMMSE filter is

$$
\begin{aligned}
& \mathbf{W}_{\mathrm{R}, \text { LRA-LMMSE }}=E\left[\mathbf{h}_{\mathrm{R}} \mathbf{z}_{\mathcal{Q}_{p}}^{T}\right] E\left[\mathbf{z}_{\mathcal{Q}_{p}} \mathbf{z}_{\mathcal{Q}_{p}}^{T}\right]^{-1} \\
& =E\left[\mathbf{h}_{\mathrm{R}}\left(\hat{\boldsymbol{\Phi}}_{\mathrm{R}} \mathbf{h}_{\mathrm{R}}+\tilde{\mathbf{n}}_{\mathrm{R}_{p}}\right)^{T}\right] E\left[\left(\hat{\boldsymbol{\Phi}}_{\mathrm{R}} \mathbf{h}_{\mathrm{R}}+\tilde{\mathbf{n}}_{\mathrm{R}_{p}}\right)\left(\hat{\boldsymbol{\Phi}}_{\mathrm{R}} \mathbf{h}_{\mathrm{R}}+\tilde{\mathbf{n}}_{\mathrm{R}_{p}}\right)^{T}\right]^{-1} \\
& =E\left[\mathbf{h}_{\mathrm{R}}\left(\mathbf{h}_{\mathrm{R}}^{T} \hat{\boldsymbol{\Phi}}_{\mathrm{R}}^{T}+\tilde{\mathbf{n}}_{\mathrm{R}_{p}}^{T}\right)\right] E\left[\left(\hat{\boldsymbol{\Phi}}_{\mathrm{R}} \mathbf{h}_{\mathrm{R}}+\tilde{\mathbf{n}}_{\mathrm{R}_{p}}\right)\left(\mathbf{h}_{\mathrm{R}}^{T} \hat{\boldsymbol{\Phi}}_{\mathrm{R}}^{T}+\tilde{\mathbf{n}}_{\mathrm{R}_{p}}^{T}\right)\right]^{-1} \\
& =E\left[\mathbf{h}_{\mathrm{R}} \mathbf{h}_{\mathrm{R}}^{T} \hat{\boldsymbol{\Phi}}_{\mathrm{R}}^{T}+\mathbf{h}_{\mathrm{R}} \tilde{\mathbf{n}}_{\mathrm{R}_{p}}^{T}\right] E\left[\hat{\boldsymbol{\Phi}}_{\mathrm{R}} \mathbf{h}_{\mathrm{R}} \mathbf{h}_{\mathrm{R}}^{T} \hat{\boldsymbol{\Phi}}_{\mathrm{R}}^{T}+\hat{\boldsymbol{\Phi}}_{\mathrm{R}} \mathbf{h}_{\mathrm{R}} \tilde{\mathbf{n}}_{\mathrm{R}_{p}}^{T}\right. \\
& \left.+\tilde{\mathbf{n}}_{\mathrm{R}_{p}} \mathbf{h}_{\mathrm{R}}^{T} \hat{\boldsymbol{\Phi}}_{\mathrm{R}}^{T}+\tilde{\mathbf{n}}_{\mathrm{R}_{p}} \tilde{\mathbf{n}}_{\mathrm{R}_{p}}^{T}\right]^{-1} \\
& =E\left[\mathbf{h}_{\mathrm{R}} \mathbf{h}_{\mathrm{R}}^{T} \hat{\boldsymbol{\Phi}}_{\mathrm{R}}^{T}\right] E\left[\hat{\boldsymbol{\Phi}}_{\mathrm{R}} \mathbf{h}_{\mathrm{R}} \mathbf{h}_{\mathrm{R}}^{T} \hat{\boldsymbol{\Phi}}_{\mathrm{R}}^{T}+\tilde{\mathbf{n}}_{\mathrm{R}_{p}} \tilde{\mathbf{n}}_{\mathrm{R}_{p}}^{T}\right]^{-1} \\
& =\mathbf{R}_{\mathbf{h}_{\mathrm{R}}} \hat{\boldsymbol{\Phi}}_{\mathrm{R}}^{T}\left(\hat{\boldsymbol{\Phi}}_{\mathrm{R}} \mathbf{R}_{\mathbf{h}_{\mathrm{R}}} \hat{\boldsymbol{\Phi}}_{\mathrm{R}}^{T}+\mathbf{C}_{\tilde{\mathbf{n}}_{\mathrm{R}_{\mathbf{p}}}}\right)^{-1} \\
& =\mathbf{R}_{\mathbf{h}_{\mathrm{R}}} \hat{\boldsymbol{\Phi}}_{\mathrm{R}}^{T} \mathbf{C}_{\mathbf{z}_{\mathcal{Q}_{p}}}^{-1} \text {, }
\end{aligned}
$$

where it is considered that $\mathbf{h}_{\mathrm{R}}$ is uncorrelated with $\tilde{\mathbf{n}}_{\mathrm{R}_{p}}$. Thus, $E\left[\mathbf{h}_{\mathrm{R}} \tilde{\mathbf{n}}_{\mathrm{R}_{p}}^{T}\right]=$ $E\left[\tilde{\mathbf{n}}_{\mathrm{R}_{p}} \mathbf{h}_{\mathrm{R}}^{T}\right]=\mathbf{0}$.

Then, the resulting LRA-LMMSE channel estimator from (4-12) is given by

$$
\hat{\mathbf{h}}_{\mathrm{R}, \text { LRA-LMMSE }}=\mathbf{W}_{\mathrm{R}, \text { LRA-LMMSE }} \mathbf{z}_{\mathcal{Q}_{p}}=\mathbf{R}_{\mathbf{h}_{\mathrm{R}}} \hat{\boldsymbol{\Phi}}_{\mathrm{R}}^{T} \mathbf{C}_{\mathbf{z}_{\mathcal{Q}_{p}}}^{-1} \mathbf{z}_{\mathcal{Q}_{p}}
$$

\section{A.3}

\section{Derivation of the MSE of the Channel Estimate}

Recalling the Mean-Squared Error problem from (4-13), we have

$$
\begin{aligned}
\mathcal{M}_{\mathrm{R}, \text { LRA-MMSE }}= & E\left[\left\|\hat{\mathbf{h}}_{\mathrm{R}, \text { LRA-MMSE }}-\mathbf{h}_{\mathrm{R}}\right\|_{2}^{2}\right] \\
= & E\left[\operatorname{tr}\left(\left(\hat{\mathbf{h}}_{\mathrm{R}, \text { LRA-MMSE }}-\mathbf{h}_{\mathrm{R}}\right)\left(\hat{\mathbf{h}}_{\mathrm{R}, \text { LRA-MMSE }}-\mathbf{h}_{\mathrm{R}}\right)^{T}\right)\right] \\
= & E\left[\operatorname{tr}\left(\left(\hat{\mathbf{h}}_{\mathrm{R}, \text { LRA-MMSE }}-\mathbf{h}_{\mathrm{R}}\right)\left(\hat{\mathbf{h}}_{\mathrm{R}, \text { LRA-MMSE }}^{T}-\mathbf{h}_{\mathrm{R}}^{T}\right)\right)\right] \\
= & E\left[\operatorname { t r } \left(\hat{\mathbf{h}}_{\mathrm{R}, \text { LRA-MMSE }} \hat{\mathbf{h}}_{\mathrm{R}, \text { LRA-MMSE }}^{T}-\hat{\mathbf{h}}_{\mathrm{R}, \text { LRA-MMSE }} \mathbf{h}_{\mathrm{R}}^{T}\right.\right. \\
& \left.\left.-\mathbf{h}_{\mathrm{R}} \hat{\mathbf{h}}_{\mathrm{R}, \text { LRA-MMSE }}^{T}+\mathbf{h}_{\mathrm{R}} \mathbf{h}_{\mathrm{R}}^{T}\right)\right] \\
= & E\left[\operatorname{tr}\left(\hat{\mathbf{h}}_{\mathrm{R}, \text { LRA-MMSE }} \hat{\mathbf{h}}_{\mathrm{R}, \text { LRA-MMSE }}^{T}-2 \hat{\mathbf{h}}_{\mathrm{R}, \text { LRA-MMSE }} \mathbf{h}_{\mathrm{R}}^{T}+\mathbf{h}_{\mathrm{R}} \mathbf{h}_{\mathrm{R}}^{T}\right)\right] .
\end{aligned}
$$

Inserting (4-12) and (4-6) into (A-6), we get 


$$
\begin{aligned}
& \mathcal{M}_{\mathrm{R}, \text { LRA-MMSE }}=E\left[\operatorname { t r } \left(\left(\mathbf{R}_{\mathbf{h}_{\mathrm{R}}} \hat{\mathbf{\Phi}}_{\mathrm{R}}^{T} \mathbf{C}_{\mathbf{z}_{\mathcal{Q}_{p}}^{-1}} \mathbf{z}_{\mathcal{Q}_{p}}\right)\left(\mathbf{R}_{\mathbf{h}_{\mathrm{R}}} \hat{\boldsymbol{\Phi}}_{\mathrm{R}}^{T} \mathbf{C}_{\mathbf{z}_{\mathcal{Q}_{p}}}^{-1} \mathbf{z}_{\mathcal{Q}_{p}}\right)^{T}\right.\right. \\
& \left.\left.-2 \mathbf{R}_{\mathbf{h}_{\mathrm{R}}} \hat{\boldsymbol{\Phi}}_{\mathrm{R}}^{T} \mathbf{C}_{\mathbf{z}_{\mathcal{Q}_{p}}}^{-1} \mathbf{z}_{\mathcal{Q}_{p}} \mathbf{h}_{\mathrm{R}}^{T}+\mathbf{h}_{\mathrm{R}} \mathbf{h}_{\mathrm{R}}^{T}\right)\right] \\
& =E\left[\operatorname { t r } \left(\mathbf{R}_{\mathbf{h}_{\mathrm{R}}} \hat{\boldsymbol{\Phi}}_{\mathrm{R}}^{T} \mathbf{C}_{\mathbf{z}_{\mathcal{Q}_{p}}}^{-1} \mathbf{z}_{\mathcal{Q}_{p}} \mathbf{z}_{\mathcal{Q}_{p}}^{T} \mathbf{C}_{\mathbf{z}_{\mathcal{Q}_{p}}}^{-1} \hat{\boldsymbol{\Phi}}_{\mathrm{R}} \mathbf{R}_{\mathbf{h}_{\mathrm{R}}}\right.\right. \\
& \left.\left.-2 \mathbf{R}_{\mathbf{h}_{\mathrm{R}}} \hat{\boldsymbol{\Phi}}_{\mathrm{R}}^{T} \mathbf{C}_{\mathbf{z}_{\mathcal{Q}_{p}}}^{-1}\left(\hat{\boldsymbol{\Phi}}_{\mathrm{R}} \mathbf{h}_{\mathrm{R}}+\tilde{\mathbf{n}}_{\mathrm{R}_{p}}\right) \mathbf{h}_{\mathrm{R}}^{T}+\mathbf{h}_{\mathrm{R}} \mathbf{h}_{\mathrm{R}}^{T}\right)\right] \\
& =E\left[\operatorname { t r } \left(\mathbf{R}_{\mathbf{h}_{\mathrm{R}}} \hat{\boldsymbol{\Phi}}_{\mathrm{R}}^{T} \mathbf{C}_{\mathbf{z}_{\mathcal{Q}_{p}}}^{-1} \mathbf{z}_{\mathcal{Q}_{p}} \mathbf{z}_{\mathcal{Q}_{p}}^{T} \mathbf{C}_{\mathbf{z}_{\mathcal{Q}_{p}}}^{-1} \hat{\boldsymbol{\Phi}}_{\mathrm{R}} \mathbf{R}_{\mathbf{h}_{\mathrm{R}}}-2 \mathbf{R}_{\mathbf{h}_{\mathrm{R}}} \hat{\boldsymbol{\Phi}}_{\mathrm{R}}^{T} \mathbf{C}_{\mathbf{z}_{\mathcal{Q}_{p}}}^{-1} \hat{\boldsymbol{\Phi}}_{\mathrm{R}} \mathbf{h}_{\mathrm{R}} \mathbf{h}_{\mathrm{R}}^{T}\right.\right. \\
& \left.\left.-2 \mathbf{R}_{\mathbf{h}_{\mathrm{R}}} \hat{\boldsymbol{\Phi}}_{\mathrm{R}}^{T} \mathbf{C}_{\mathbf{z}_{\mathcal{Q}_{p}}^{-1}}^{-1} \tilde{\mathbf{n}}_{\mathrm{R}_{p}} \mathbf{h}_{\mathrm{R}}^{T}+\mathbf{h}_{\mathrm{R}} \mathbf{h}_{\mathrm{R}}^{T}\right)\right] \\
& =\operatorname{tr}\left(\mathbf{R}_{\mathbf{h}_{\mathrm{R}}} \hat{\boldsymbol{\Phi}}_{\mathrm{R}}^{T} \mathbf{C}_{\mathbf{z}_{\mathcal{Q}_{p}}}^{-1} \mathbf{C}_{\mathbf{z}_{\mathcal{Q}_{p}}} \mathbf{C}_{\mathbf{z}_{\mathcal{Q}_{p}}}^{-1} \hat{\boldsymbol{\Phi}}_{\mathrm{R}} \mathbf{R}_{\mathbf{h}_{\mathrm{R}}}-2 \mathbf{R}_{\mathbf{h}_{\mathrm{R}}} \hat{\boldsymbol{\Phi}}_{\mathrm{R}}^{T} \mathbf{C}_{\mathbf{z}_{\mathcal{Q}_{p}}}^{-1} \hat{\boldsymbol{\Phi}}_{\mathrm{R}} \mathbf{R}_{\mathbf{h}_{\mathrm{R}}}+\mathbf{R}_{\mathbf{h}_{\mathrm{R}}}\right) \\
& =\operatorname{tr}\left(\mathbf{R}_{\mathbf{h}_{\mathrm{R}}} \hat{\boldsymbol{\Phi}}_{\mathrm{R}}^{T} \mathbf{C}_{\mathbf{z}_{\mathcal{Q}_{p}}}^{-1} \hat{\boldsymbol{\Phi}}_{\mathrm{R}} \mathbf{R}_{\mathbf{h}_{\mathrm{R}}}-2 \mathbf{R}_{\mathbf{h}_{\mathrm{R}}} \hat{\boldsymbol{\Phi}}_{\mathrm{R}}^{T} \mathbf{C}_{\mathbf{z}_{\mathcal{Q}_{p}}}^{-1} \hat{\boldsymbol{\Phi}}_{\mathrm{R}} \mathbf{R}_{\mathbf{h}_{\mathrm{R}}}+\mathbf{R}_{\mathbf{h}_{\mathrm{R}}}\right) \\
& =\operatorname{tr}\left(\mathbf{R}_{\mathbf{h}_{\mathrm{R}}}-\mathbf{R}_{\mathbf{h}_{\mathrm{R}}} \hat{\boldsymbol{\Phi}}_{\mathrm{R}}^{T} \mathbf{C}_{\mathbf{z}_{\mathcal{Q}_{p}}}^{-1} \hat{\boldsymbol{\Phi}}_{\mathrm{R}} \mathbf{R}_{\mathbf{h}_{\mathrm{R}}}\right) \text {, }
\end{aligned}
$$

where it is considered that $\mathbf{h}_{\mathrm{R}}$ is uncorrelated with $\tilde{\mathbf{n}}_{\mathrm{R}_{p}}$. Thus, $E\left[\tilde{\mathbf{n}}_{\mathrm{R}_{p}} \mathbf{h}_{\mathrm{R}}^{T}\right]=\mathbf{0}$. 
B

\section{Linear Detection Derivations}

\section{B.1}

Derivation of the Cross-Correlation Matrix between Received and Transmitted Data Signals

The cross-correlation matrix between $\mathbf{z}_{\mathrm{R}}$ and $\mathbf{x}_{\mathrm{R}}$ from (5-5) is calculated as follows

$$
\begin{aligned}
\mathbf{C}_{\mathbf{z}_{\mathrm{R}} \mathbf{x}_{\mathrm{R}}} & =E\left[\mathbf{z}_{\mathrm{R}} \mathbf{x}_{\mathrm{R}}^{T}\right]=E\left[\left(\mathbf{B} \mathbf{y}_{\mathrm{R}}\right) \mathbf{x}_{\mathrm{R}}^{T}\right]=E\left[\left(\mathbf{B}\left(\mathbf{H}_{\mathrm{R}} \mathbf{x}_{\mathrm{R}}+\mathbf{n}_{\mathrm{R}}\right)\right) \mathbf{x}_{\mathrm{R}}^{T}\right] \\
& =E\left[\left(\left(\mathbf{B} \mathbf{H}_{\mathrm{R}} \mathbf{x}_{\mathrm{R}}+\mathbf{B} \mathbf{n}_{\mathrm{R}}\right)\right) \mathbf{x}_{\mathrm{R}}^{T}\right]=E\left[\mathbf{B} \mathbf{H}_{\mathrm{R}} \mathbf{x}_{\mathrm{R}} \mathbf{x}_{\mathrm{R}}^{T}+\mathbf{B} \mathbf{n}_{\mathrm{R}} \mathbf{x}_{\mathrm{R}}^{T}\right] \\
& =\mathbf{B} \mathbf{H}_{\mathrm{R}} \mathbf{C}_{\mathbf{x}_{\mathrm{R}}},
\end{aligned}
$$

where it is considered that $\mathbf{x}_{\mathrm{R}}$ is uncorrelated with $\mathbf{n}_{\mathrm{R}}$. Thus, $E\left[\mathbf{n}_{\mathrm{R}} \mathbf{x}_{\mathrm{R}}^{T}\right]=\mathbf{0}$.

\section{B.2}

\section{Derivation of the Auto-Correlation of the Received Data Signal}

The auto-correlation of $\mathbf{z}_{\mathrm{R}}$ from (5-6) is calculated as follows

$$
\begin{aligned}
\mathbf{C}_{\mathbf{z}_{\mathrm{R}}} & =E\left[\mathbf{z}_{\mathrm{R}} \mathbf{z}_{\mathrm{R}}^{T}\right]=E\left[\left(\mathbf{B} \mathbf{y}_{\mathrm{R}}\right)\left(\mathbf{B} \mathbf{y}_{\mathrm{R}}\right)^{T}\right]=E\left[\mathbf{B} \mathbf{y}_{\mathrm{R}} \mathbf{y}_{\mathrm{R}}^{T} \mathbf{B}^{T}\right] \\
& =E\left[\mathbf{B}\left(\mathbf{H}_{\mathrm{R}} \mathbf{x}_{\mathrm{R}}+\mathbf{n}_{\mathrm{R}}\right)\left(\mathbf{H}_{\mathrm{R}} \mathbf{x}_{\mathrm{R}}+\mathbf{n}_{\mathrm{R}}\right)^{T} \mathbf{B}^{T}\right] \\
& =E\left[\mathbf{B}\left(\mathbf{H}_{\mathrm{R}} \mathbf{x}_{\mathrm{R}}+\mathbf{n}_{\mathrm{R}}\right)\left(\mathbf{x}_{\mathrm{R}}^{T} \mathbf{H}_{\mathrm{R}}^{T}+\mathbf{n}_{\mathrm{R}}^{T}\right) \mathbf{B}^{T}\right] \\
& =E\left[\left(\mathbf{B} \mathbf{H}_{\mathrm{R}} \mathbf{x}_{\mathrm{R}}+\mathbf{B} \mathbf{n}_{\mathrm{R}}\right)\left(\mathbf{x}_{\mathrm{R}}^{T} \mathbf{H}_{\mathrm{R}}^{T} \mathbf{B}^{T}+\mathbf{n}_{\mathrm{R}}^{T} \mathbf{B}^{T}\right)\right] \\
& =E\left[\mathbf{B} \mathbf{H}_{\mathrm{R}} \mathbf{x}_{\mathrm{R}} \mathbf{x}_{\mathrm{R}}^{T} \mathbf{H}_{\mathrm{R}}^{T} \mathbf{B}^{T}+\mathbf{B} \mathbf{H}_{\mathrm{R}} \mathbf{x}_{\mathrm{R}} \mathbf{n}_{\mathrm{R}}^{T} \mathbf{B}^{T}+\mathbf{B} \mathbf{n}_{\mathrm{R}} \mathbf{x}_{\mathrm{R}}^{T} \mathbf{H}_{\mathrm{R}}^{T} \mathbf{B}^{T}+\mathbf{B} \mathbf{n}_{\mathrm{R}} \mathbf{n}_{\mathrm{R}}^{T} \mathbf{B}^{T}\right] \\
& =\mathbf{B} \mathbf{H}_{\mathrm{R}} \mathbf{C}_{\mathbf{x}_{\mathrm{R}}} \mathbf{H}_{\mathrm{R}}^{T} \mathbf{B}^{T}+\mathbf{B} \mathbf{C}_{\mathbf{n}_{\mathrm{R}}} \mathbf{B}^{T},
\end{aligned}
$$

where it is considered that $\mathbf{x}_{\mathrm{R}}$ is uncorrelated with $\mathbf{n}_{\mathrm{R}}$. Thus, $E\left[\mathbf{x}_{\mathrm{R}} \mathbf{n}_{\mathrm{R}}^{T}\right]=$ $E\left[\mathbf{n}_{\mathrm{R}} \mathbf{x}_{\mathrm{R}}^{T}\right]=\mathbf{0}$. 


\section{B.3}

Derivation of the MSE between the Transmitted Symbol and the Filter Output for the Robust Detector

The MSE between the transmitted symbol $\mathbf{x}_{\mathrm{R}}$ and the filter output $\hat{\mathbf{x}}_{\mathrm{R}}$ for the robust detector from (5-11) is calculated as follows

$$
\begin{aligned}
& E\left[\left\|\mathbf{x}_{\mathrm{R}}-\mathbf{G}_{\mathrm{R}}^{T} \mathbf{z}_{\mathcal{Q}_{r}}\right\|_{2}^{2}\right]=E\left[\operatorname{tr}\left(\left(\mathbf{x}_{\mathrm{R}}-\mathbf{G}_{\mathrm{R}}^{T} \mathbf{z}_{\mathcal{Q}_{r}}\right)\left(\mathbf{x}_{\mathrm{R}}-\mathbf{G}_{\mathrm{R}}^{T} \mathbf{z}_{\mathcal{Q}_{r}}\right)^{T}\right)\right] \\
& =E\left[\operatorname{tr}\left(\left(\mathbf{x}_{\mathrm{R}}-\mathbf{G}_{\mathrm{R}}^{T} \mathbf{z}_{\mathcal{Q}_{r}}\right)\left(\mathbf{x}_{\mathrm{R}}^{T}-\mathbf{z}_{\mathcal{Q}_{r}}^{T} \mathbf{G}_{\mathrm{R}}\right)\right)\right] \\
& =E\left[\operatorname{tr}\left(\mathbf{x}_{\mathrm{R}} \mathbf{x}_{\mathrm{R}}^{T}-\mathbf{x}_{\mathrm{R}} \mathbf{z}_{\mathcal{Q}_{r}}^{T} \mathbf{G}_{\mathrm{R}}-\mathbf{G}_{\mathrm{R}}^{T} \mathbf{z}_{\mathcal{Q}_{r}} \mathbf{x}_{\mathrm{R}}^{T}+\mathbf{G}_{\mathrm{R}}^{T} \mathbf{z}_{\mathcal{Q}_{r}} \mathbf{z}_{\mathcal{Q}_{r}}^{T} \mathbf{G}_{\mathrm{R}}\right)\right] \\
& =E\left[\operatorname{tr}\left(\mathbf{x}_{\mathrm{R}} \mathbf{x}_{\mathrm{R}}^{T}-2 \mathbf{G}_{\mathrm{R}}^{T} \mathbf{z}_{\mathcal{Q}_{r}} \mathbf{x}_{\mathrm{R}}^{T}+\mathbf{G}_{\mathrm{R}}^{T} \mathbf{z}_{\mathcal{Q}_{r}} \mathbf{z}_{\mathcal{Q}_{r}}^{T} \mathbf{G}_{\mathrm{R}}\right)\right] \\
& =\operatorname{tr}\left(\mathbf{C}_{\mathbf{x}_{\mathrm{R}}}-2 \mathbf{G}_{\mathrm{R}}^{T} \mathbf{C}_{\mathbf{z}_{\mathcal{Q}_{r}} \mathbf{x}_{\mathrm{R}}}+\mathbf{G}_{\mathrm{R}}^{T} \mathbf{C}_{\mathbf{z}_{\mathcal{Q}_{r}}} \mathbf{G}_{\mathrm{R}}\right) \\
& =\operatorname{tr}\left(\frac{1}{2} \mathbf{I}_{2 N_{t}}-2 \mathbf{G}_{\mathrm{R}}^{T} \mathbf{C}_{\mathbf{z}_{\mathcal{Q}_{r}} \mathbf{x}_{\mathrm{R}}}+\mathbf{G}_{\mathrm{R}}^{T} \mathbf{C}_{\mathbf{z}_{\mathcal{Q}_{r}}} \mathbf{G}_{\mathrm{R}}\right) \text {, }
\end{aligned}
$$

where due to the real-valued notation of the system $\mathbf{C}_{\mathbf{x}_{\mathrm{R}}}=E\left[\mathbf{x}_{\mathrm{R}} \mathbf{x}_{\mathrm{R}}^{T}\right]=\frac{1}{2} \mathbf{I}_{2 N_{t}}$.

\section{B.4}

\section{Derivation of the Robust Cross-Correlation Matrix between Received and Transmitted Data Signals}

The cross-correlation matrix between $\mathbf{z}_{\mathrm{R}_{r}}$ and $\mathbf{x}_{\mathrm{R}}$ from (5-14) is calculated as follows

$$
\begin{aligned}
\mathbf{C}_{\mathbf{z}_{\mathrm{R}} \mathbf{x}_{\mathrm{R}}} & =E\left[\mathbf{z}_{\mathrm{R}_{r}} \mathbf{x}_{\mathrm{R}}^{T}\right]=E\left[\left(\mathbf{B} \hat{\mathbf{H}}_{\mathrm{R}} \mathbf{x}_{\mathrm{R}}+\mathbf{B} \tilde{\mathbf{X}}_{\mathrm{R}} \boldsymbol{\varepsilon}_{\mathrm{R}}+\mathbf{B} \mathbf{n}_{\mathrm{R}_{d}}\right) \mathbf{x}_{\mathrm{R}}^{T}\right] \\
& =E\left[\mathbf{B} \hat{\mathbf{H}}_{\mathrm{R}} \mathbf{x}_{\mathrm{R}} \mathbf{x}_{\mathrm{R}}^{T}\right]=\mathbf{B} \hat{\mathbf{H}}_{\mathrm{R}} \mathbf{C}_{\mathbf{x}_{\mathrm{R}}},
\end{aligned}
$$

where it is considered that $\mathbf{x}_{\mathrm{R}}$ is uncorrelated with $\mathbf{n}_{\mathrm{R}_{d}}$ and $\varepsilon_{\mathrm{R}}$.

\section{B.5}

\section{Derivation of the Robust Auto-Correlation Received Data Signal}

The auto-correlation of $\mathbf{z}_{\mathrm{R}_{r}}$ from (5-16) s calculated as follows

$$
\begin{aligned}
\mathbf{C}_{\mathbf{z}_{\mathrm{R}}} & =E\left[\mathbf{z}_{\mathrm{R}_{r}} \mathbf{z}_{\mathrm{R}_{r}}^{T}\right] \\
& =E\left[\left(\mathbf{B} \hat{\mathbf{H}}_{\mathrm{R}} \mathbf{x}_{\mathrm{R}}+\mathbf{B} \tilde{\mathbf{X}}_{\mathrm{R}} \boldsymbol{\varepsilon}_{\mathrm{R}}+\mathbf{B} \mathbf{n}_{\mathrm{R}_{d}}\right)\left(\mathbf{B} \hat{\mathbf{H}}_{\mathrm{R}} \mathbf{x}_{\mathrm{R}}+\mathbf{B} \tilde{\mathbf{X}}_{\mathrm{R}} \boldsymbol{\varepsilon}_{\mathrm{R}}+\mathbf{B} \mathbf{n}_{\mathrm{R}_{d}}\right)^{T}\right] \\
& =E\left[\left(\mathbf{B} \hat{\mathbf{H}}_{\mathrm{R}} \mathbf{x}_{\mathrm{R}}+\mathbf{B} \tilde{\mathbf{X}}_{\mathrm{R}} \boldsymbol{\varepsilon}_{\mathrm{R}}+\mathbf{B} \mathbf{n}_{\mathrm{R}_{d}}\right)\left(\mathbf{x}_{\mathrm{R}}^{T} \hat{\mathbf{H}}_{\mathrm{R}}^{T} \mathbf{B}^{T}+\boldsymbol{\varepsilon}_{\mathrm{R}}^{T} \tilde{\mathbf{X}}_{\mathrm{R}}^{T} \mathbf{B}^{T}+\mathbf{n}_{\mathrm{R}_{d}}^{T} \mathbf{B}^{T}\right)\right] \\
& =E\left[\mathbf{B} \hat{\mathbf{H}}_{\mathrm{R}} \mathbf{x}_{\mathrm{R}} \mathbf{x}_{\mathrm{R}}^{T} \hat{\mathbf{H}}_{\mathrm{R}}^{T} \mathbf{B}^{T}+\mathbf{B} \tilde{\mathbf{X}}_{\mathrm{R}} \boldsymbol{\varepsilon}_{\mathrm{R}} \varepsilon_{\mathrm{R}}^{T} \tilde{\mathbf{X}}_{\mathrm{R}}^{T} \mathbf{B}^{T}+\mathbf{B} \mathbf{n}_{\mathrm{R}_{d}} \mathbf{n}_{\mathrm{R}_{d}}^{T} \mathbf{B}^{T}\right] \\
& =\mathbf{B} \hat{\mathbf{H}}_{\mathrm{R}} \mathbf{C}_{\mathbf{x}_{\mathrm{R}}} \hat{\mathbf{H}}_{\mathrm{R}}^{T} \mathbf{B}^{T}+\mathbf{B} E\left[\tilde{\mathbf{X}}_{\mathrm{R}} E\left[\varepsilon_{\mathrm{R}} \varepsilon_{\mathrm{R}}^{T}\right] \tilde{\mathbf{X}}_{\mathrm{R}}^{T}\right] \mathbf{B}^{T}+\mathbf{B} \mathbf{C}_{\mathbf{n}_{\mathrm{R}_{d}}} \mathbf{B}^{T} \\
& =\frac{1}{2} \mathbf{B} \hat{\mathbf{H}}_{\mathrm{R}} \hat{\mathbf{H}}_{\mathrm{R}}^{T} \mathbf{B}^{T}+\mathbf{B} \boldsymbol{\Gamma}_{\mathrm{R}} \mathbf{B}^{T}+\frac{\sigma_{n}^{2}}{2} \mathbf{B B}^{T},
\end{aligned}
$$


where due to the real-valued notation of the system $\mathbf{C}_{\mathbf{x}_{\mathrm{R}}}=E\left[\mathbf{x}_{\mathrm{R}} \mathbf{x}_{\mathrm{R}}^{T}\right]=\frac{1}{2} \mathbf{I}_{2 N_{t}}$ and $\mathbf{C}_{\mathbf{n}_{\mathrm{R}}}=E\left[\mathbf{n}_{\mathrm{R}} \mathbf{n}_{\mathrm{R}}^{T}\right]=\frac{\sigma_{n}^{2}}{2} \mathbf{I}_{2 N_{r}}$. Moreover, by considering that $\tilde{\mathbf{X}}_{\mathrm{R}}$ and $\boldsymbol{\varepsilon}_{\mathrm{R}}$ are independent from each other, we have $\boldsymbol{\Gamma}_{\mathrm{R}}=E\left[\tilde{\mathbf{X}}_{\mathrm{R}} E\left[\varepsilon_{\mathrm{R}} \varepsilon_{\mathrm{R}}^{T}\right] \tilde{\mathbf{X}}_{\mathrm{R}}^{T}\right]$. 
C

\section{Sum Rate Derivations}

\section{C.1}

\section{Derivation of the Quantization Noise Covariance Matrix}

The quantization noise covariance matrix from (6-4) is calculated as follows

$$
\begin{aligned}
& \mathbf{C}_{\mathbf{n}_{\mathrm{R}_{q, d}}}=E\left[\left(\mathbf{z}_{\mathcal{Q}_{d}}-\mathbf{A}_{\mathrm{R}_{d}} \mathbf{z}_{\mathrm{R}_{d}}\right)\left(\mathbf{z}_{\mathcal{Q}_{d}}-\mathbf{A}_{\mathrm{R}_{d}} \mathbf{z}_{\mathrm{R}_{d}}\right)^{T}\right] \\
& =E\left[\left(\mathbf{z}_{\mathcal{Q}_{d}}-\mathbf{A}_{\mathrm{R}_{d}} \mathbf{z}_{\mathrm{R}_{d}}\right)\left(\mathbf{z}_{\mathcal{Q}_{d}}^{T}-\mathbf{z}_{\mathrm{R}_{d}}^{T} \mathbf{A}_{\mathrm{R}_{d}}\right)\right] \\
& =E\left[\mathbf{z}_{\mathcal{Q}_{d}} \mathbf{z}_{\mathcal{Q}_{d}}^{T}-\mathbf{z}_{\mathcal{Q}_{d}} \mathbf{z}_{\mathrm{R}_{d}}^{T} \mathbf{A}_{\mathrm{R}_{d}}-\mathbf{A}_{\mathrm{R}_{d}} \mathbf{z}_{\mathrm{R}_{d}} \mathbf{z}_{\mathcal{Q}_{d}}^{T}+\mathbf{A}_{\mathrm{R}_{d}} \mathbf{z}_{\mathrm{R}_{d}} \mathbf{z}_{\mathrm{R}_{d}}^{T} \mathbf{A}_{\mathrm{R}_{d}}\right] \\
& =\mathrm{C}_{\mathbf{z}_{\mathcal{Q}_{d}}}-\mathrm{C}_{\mathbf{z}_{\mathcal{Q}_{d}} \mathbf{z}_{d}} \mathbf{A}_{\mathrm{R}_{d}}-\mathbf{A}_{\mathrm{R}_{d}} \mathbf{C}_{\mathbf{z}_{\mathrm{R}_{d}} \mathbf{z}_{\mathcal{Q}_{d}}}+\mathbf{A}_{\mathrm{R}_{d}} \mathbf{C}_{\mathbf{z}_{\mathrm{R}_{d}}} \mathbf{A}_{\mathrm{R}_{d}}
\end{aligned}
$$

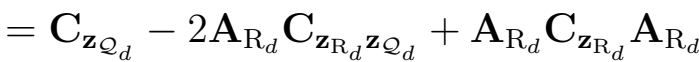

$$
\begin{aligned}
& =\mathbf{C}_{\mathbf{z}_{\mathcal{Q}_{d}}}-2 \mathbf{A}_{\mathrm{R}_{d}} \mathbf{C}_{\mathbf{z}_{d}} \mathbf{A}_{\mathrm{R}_{d}}+\mathbf{A}_{\mathrm{R}_{d}} \mathbf{C}_{\mathbf{z}_{d}} \mathbf{A}_{\mathrm{R}_{d}} \\
& =\mathbf{C}_{\mathbf{z}_{\mathcal{Q}_{d}}}-\mathbf{A}_{\mathrm{R}_{d}} \mathbf{C}_{\mathbf{z}_{\mathrm{R}_{d}}} \mathbf{A}_{\mathrm{R}_{d}} \text {. }
\end{aligned}
$$

Note that since $\mathbf{A}_{\mathrm{R}_{d}}$ is a diagonal matrix, $\mathbf{A}_{\mathrm{R}_{d}}=\mathbf{A}_{\mathrm{R}_{d}}^{T}$. Moreover, it holds $\mathbf{C}_{\mathbf{z}_{\mathrm{R}_{d}}}=\mathbf{C}_{\mathbf{z}_{\mathrm{R}_{d}}}^{T}$ because it is a real-valued covariance matrix.

\section{2}

Derivation of the Ergodic Achievable Rate for Real-Valued User Channel

First, let us recall the received signal of the index $k$, with $k \in\left[1,2 N_{t}\right]$, given by (6-3)

$$
\begin{aligned}
\hat{\mathbf{x}}_{\mathrm{R}_{k}}= & \underbrace{\mathbf{g}_{\mathrm{R}_{k}}^{T} \mathbf{A}_{\mathrm{R}_{d}} \mathbf{B} \hat{\mathbf{h}}_{\mathrm{R}_{k}} \mathbf{x}_{\mathrm{R}_{k}}}_{\text {desired signal }}+\underbrace{\mathbf{g}_{\mathrm{R}_{k}}^{T} \sum_{i \neq k}^{K} \mathbf{A}_{\mathrm{R}_{d}} \mathbf{B} \hat{\mathbf{h}}_{\mathrm{R}_{i}} \mathbf{x}_{\mathrm{R}_{i}}}_{\text {interference }}+\underbrace{\mathbf{g}_{\mathrm{R}_{k}}^{T} \sum_{i=1}^{K} \mathbf{A}_{\mathrm{R}_{d}} \mathbf{B} \boldsymbol{\varepsilon}_{\mathrm{R}_{i}} \mathbf{x}_{\mathrm{R}_{i}}}_{\text {channel estimation error }} \\
& +\underbrace{\mathbf{g}_{\mathrm{R}_{k}}^{T} \mathbf{A}_{\mathrm{R}_{d}} \mathbf{B} \mathbf{n}_{\mathrm{R}_{d}}}_{\text {AWGN noise }}+\underbrace{\mathbf{g}_{\mathrm{R}_{k}}^{T} \mathbf{n}_{\mathrm{R}_{q, d}}}_{\text {quant. noise }},
\end{aligned}
$$

where $\mathbf{g}_{\mathrm{R}_{k}}^{T}$ is the $k$ th row of $\mathbf{G}_{\mathrm{R}}$ and $\hat{\mathbf{h}}_{\mathrm{R}_{k}}$ is the $k$ th column of $\hat{\mathbf{H}}_{\mathrm{R}}$. Moreover, $\boldsymbol{\varepsilon}_{\mathrm{R}_{i}}$ is the $i$ th column of the matrix $\mathcal{E}_{\mathrm{R}}$.

Assuming Gaussian signaling, the ergodic achievable rate per channel $k$, for the real-valued notation, is lower bounded by 


$$
I_{\mathrm{R}_{k}}=E\left[\frac{1}{2} \log _{2}\left(1+\operatorname{SINR}_{k}\right)\right] .
$$

Then, the sum-rate is described as

$$
I_{\mathrm{R}}=\sum_{k=1}^{K} I_{\mathrm{R}_{k}}=\sum_{k=1}^{K} E\left[\frac{1}{2} \log _{2}\left(1+\operatorname{SINR}_{k}\right)\right],
$$

where

$$
\operatorname{SINR}_{k}=\frac{E\left[\left|T_{1}\right|^{2}\right]}{\sum_{i \neq k}^{K} E\left[\left|T_{2, i}\right|^{2}\right]+\sum_{i=1}^{K} E\left[\left|T_{3, i}\right|^{2}\right]+E\left[\left|T_{4}\right|^{2}\right]+E\left[\left|T_{5}\right|^{2}\right]} .
$$

In the expression above, the term

$$
T_{1}=\mathbf{g}_{\mathrm{R}_{k}}^{T} \mathbf{A}_{\mathrm{R}_{d}} \mathbf{B} \hat{\mathbf{h}}_{\mathrm{R}_{k}} \mathbf{x}_{\mathrm{R}_{k}}
$$

represents the desired signal, while the parameter

$$
T_{2, i}=\mathbf{g}_{\mathrm{R}_{k}}^{T} \mathbf{A}_{\mathrm{R}_{d}} \mathbf{B} \hat{\mathbf{h}}_{\mathrm{R}_{i}} \mathbf{x}_{\mathrm{R}_{i}} \text {, for } i \neq k, i=1, \ldots, K,
$$

is the interference caused by user $i$ in user $k$. The quantity

$$
T_{3, i}=\mathbf{g}_{\mathrm{R}_{k}}^{T} \mathbf{A}_{\mathrm{R}_{d}} \mathbf{B} \varepsilon_{\mathrm{R}_{i}} \mathbf{x}_{\mathrm{R}_{i}}, \text { for } i=k, i=1, \ldots, K,
$$

refers to the channel estimation error, whilst

$$
T_{4}=\mathbf{g}_{\mathrm{R}_{k}}^{T} \mathbf{A}_{\mathrm{R}_{d}} \mathbf{B n}_{\mathrm{R}_{d}}
$$

corresponds to the AWGN noise and

$$
T_{5}=\mathbf{g}_{\mathrm{R}_{k}}^{T} \mathbf{n}_{\mathrm{R}_{q, d}}
$$

relates to the quantizer noise.

The mean-square values of the terms $T_{1}, T_{2, i}, T_{3, i}, T_{4}$ and $T_{5}$ are computed as follows:

$$
\begin{aligned}
E\left[\left|T_{1}\right|^{2}\right] & =E\left[\left(\mathbf{g}_{\mathrm{R}_{k}}^{T} \mathbf{A}_{\mathrm{R}_{d}} \mathbf{B} \hat{\mathbf{h}}_{\mathrm{R}_{k}} \mathbf{x}_{\mathrm{R}_{k}}\right)\left(\mathbf{g}_{\mathrm{R}_{k}}^{T} \mathbf{A}_{\mathrm{R}_{d}} \mathbf{B} \hat{\mathbf{h}}_{\mathrm{R}_{k}} \mathbf{x}_{\mathrm{R}_{k}}\right)^{T}\right] \\
& =E\left[\mathbf{g}_{\mathrm{R}_{k}}^{T} \mathbf{A}_{\mathrm{R}_{d}} \mathbf{B} \hat{\mathbf{h}}_{\mathrm{R}_{k}} \mathbf{x}_{\mathrm{R}_{k}} \mathbf{x}_{\mathrm{R}_{k}}^{T} \hat{\mathbf{h}}_{\mathrm{R}_{k}}^{T} \mathbf{B}^{T} \mathbf{A}_{\mathrm{R}_{d}}^{T} \mathbf{g}_{\mathrm{R}_{k}}\right] \\
& =\mathbf{g}_{\mathrm{R}_{k}}^{T} \mathbf{A}_{\mathrm{R}_{d}} \mathbf{B} \hat{\mathbf{h}}_{\mathrm{R}_{k}} \mathbf{C}_{\mathbf{x}_{\mathrm{R}}} \hat{\mathbf{h}}_{\mathrm{R}_{k}}^{T} \mathbf{B}^{T} \mathbf{A}_{\mathrm{R}_{d}}^{T} \mathbf{g}_{\mathrm{R}_{k}} \\
& =\frac{1}{2} \mathbf{g}_{\mathrm{R}_{k}}^{T} \mathbf{A}_{\mathrm{R}_{d}} \mathbf{B} \hat{\mathbf{h}}_{\mathrm{R}_{k}} \hat{\mathbf{h}}_{\mathrm{R}_{k}}^{T} \mathbf{B}^{T} \mathbf{A}_{\mathrm{R}_{d}}^{T} \mathbf{g}_{\mathrm{R}_{k}} \\
& =\frac{1}{2}\left|\mathbf{g}_{\mathrm{R}_{k}}^{T} \mathbf{A}_{\mathrm{R}_{d}} \mathbf{B} \hat{\mathbf{h}}_{\mathrm{R}_{k}}\right|^{2} \\
& =\frac{1}{2}\left|\mathbf{d}_{\mathrm{R}_{k}} \hat{\mathbf{h}}_{\mathrm{R}_{k}}\right|^{2}
\end{aligned}
$$




$$
\begin{aligned}
& E\left[\left|T_{2, i}\right|^{2}\right]=E\left[\left(\mathbf{g}_{\mathrm{R}_{k}}^{T} \mathbf{A}_{\mathrm{R}_{d}} \mathbf{B} \hat{\mathbf{h}}_{\mathrm{R}_{i}} \mathbf{x}_{\mathrm{R}_{i}}\right)\left(\mathbf{g}_{\mathrm{R}_{k}}^{T} \mathbf{A}_{\mathrm{R}_{d}} \mathbf{B} \hat{\mathbf{h}}_{\mathrm{R}_{i}} \mathbf{x}_{\mathrm{R}_{i}}\right)^{T}\right] \\
& =E\left[\mathbf{g}_{\mathrm{R}_{k}}^{T} \mathbf{A}_{\mathrm{R}_{d}} \mathbf{B} \hat{\mathbf{h}}_{\mathrm{R}_{i}} \mathbf{x}_{\mathrm{R}_{i}} \mathbf{x}_{\mathrm{R}_{i}}^{T} \hat{\mathbf{h}}_{\mathrm{R}_{i}}^{T} \mathbf{B}^{T} \mathbf{A}_{\mathrm{R}_{d}}^{T} \mathbf{g}_{\mathrm{R}_{k}}\right] \\
& =\mathbf{g}_{\mathrm{R}_{k}}^{T} \mathbf{A}_{\mathrm{R}_{d}} \mathbf{B} \hat{\mathbf{h}}_{\mathrm{R}_{i}} \mathbf{C}_{\mathbf{x}_{\mathrm{R}}} \hat{\mathbf{h}}_{\mathrm{R}_{i}}^{T} \mathbf{B}^{T} \mathbf{A}_{\mathrm{R}_{d}}^{T} \mathbf{g}_{\mathrm{R}_{k}} \\
& =\frac{1}{2} \mathbf{g}_{\mathrm{R}_{k}}^{T} \mathbf{A}_{\mathrm{R}_{d}} \mathbf{B} \hat{\mathbf{h}}_{\mathrm{R}_{i}} \hat{\mathbf{h}}_{\mathrm{R}_{i}}^{T} \mathbf{B}^{T} \mathbf{A}_{\mathrm{R}_{d}}^{T} \mathbf{g}_{\mathrm{R}_{k}} \\
& =\frac{1}{2}\left|\mathbf{g}_{\mathrm{R}_{k}}^{T} \mathbf{A}_{\mathrm{R}_{d}} \mathbf{B} \hat{\mathbf{h}}_{\mathrm{R}_{i}}\right|^{2} \\
& =\frac{1}{2}\left|\mathbf{d}_{\mathrm{R}_{k}} \hat{\mathbf{h}}_{\mathrm{R}_{i}}\right|^{2} \text {, } \\
& E\left[\left|T_{3, i}\right|^{2}\right]=E\left[\left(\mathbf{g}_{\mathrm{R}_{k}}^{T} \mathbf{A}_{\mathrm{R}_{d}} \mathbf{B} \varepsilon_{\mathrm{R}_{i}} \mathbf{x}_{\mathrm{R}_{i}}\right)\left(\mathbf{g}_{\mathrm{R}_{k}}^{T} \mathbf{A}_{\mathrm{R}_{d}} \mathbf{B} \varepsilon_{\mathrm{R}_{i}} \mathbf{x}_{\mathrm{R}_{i}}\right)^{T}\right] \\
& =E\left[\mathbf{g}_{\mathrm{R}_{k}}^{T} \mathbf{A}_{\mathrm{R}_{d}} \mathbf{B} \varepsilon_{\mathrm{R}_{i}} \mathbf{x}_{\mathrm{R}_{i}} \mathbf{x}_{\mathrm{R}_{i}}^{T} \boldsymbol{\varepsilon}_{\mathrm{R}_{i}}^{T} \mathbf{B}^{T} \mathbf{A}_{\mathrm{R}_{d}}^{T} \mathbf{g}_{\mathrm{R}_{k}}\right] \\
& =\mathbf{g}_{\mathrm{R}_{k}}^{T} \mathbf{A}_{\mathrm{R}_{d}} \mathbf{B} \boldsymbol{\varepsilon}_{\mathrm{R}_{i}} \mathbf{C}_{\mathbf{x}_{\mathrm{R}}} \varepsilon_{\mathrm{R}_{i}}^{T} \mathbf{B}^{T} \mathbf{A}_{\mathrm{R}_{d}}^{T} \mathbf{g}_{\mathrm{R}_{k}} \\
& =\frac{1}{2} \mathbf{g}_{\mathrm{R}_{k}}^{T} \mathbf{A}_{\mathrm{R}_{d}} \mathbf{B} \boldsymbol{\varepsilon}_{\mathrm{R}_{i}} \varepsilon_{\mathrm{R}_{i}}^{T} \mathbf{B}^{T} \mathbf{A}_{\mathrm{R}_{d}}^{T} \mathbf{g}_{\mathrm{R}_{k}} \\
& =\frac{1}{2}\left|\mathbf{g}_{\mathrm{R}_{k}}^{T} \mathbf{A}_{\mathrm{R}_{d}} \mathbf{B} \varepsilon_{\mathrm{R}_{i}}\right|^{2} \\
& =\frac{1}{2}\left|\mathbf{d}_{\mathrm{R}_{k}} \boldsymbol{\varepsilon}_{\mathrm{R}_{i}}\right|^{2} \text {, } \\
& E\left[\left|T_{4}\right|^{2}\right]=E\left[\left(\mathbf{g}_{\mathrm{R}_{k}}^{T} \mathbf{A}_{\mathrm{R}_{d}} \mathbf{B n}_{\mathrm{R}_{d}}\right)\left(\mathbf{g}_{\mathrm{R}_{k}}^{T} \mathbf{A}_{\mathrm{R}_{d}} \mathbf{B n}_{\mathrm{R}_{d}}\right)^{T}\right] \\
& =E\left[\mathbf{g}_{\mathrm{R}_{k}}^{T} \mathbf{A}_{\mathrm{R}_{d}} \mathbf{B n}_{\mathrm{R}_{d}} \mathbf{n}_{\mathrm{R}_{d}}^{T} \mathbf{B}^{T} \mathbf{A}_{\mathrm{R}_{d}}^{T} \mathbf{g}_{\mathrm{R}_{k}}\right] \\
& =\mathbf{g}_{\mathrm{R}_{k}}^{T} \mathbf{A}_{\mathrm{R}_{d}} \mathbf{B C}_{\mathbf{n}_{\mathrm{R}_{\mathbf{d}}}} \mathbf{B}^{T} \mathbf{A}_{\mathrm{R}_{d}}^{T} \mathbf{g}_{\mathrm{R}_{k}} \\
& =\frac{\sigma_{n}^{2}}{2} \mathbf{g}_{\mathrm{R}_{k}}^{T} \mathbf{A}_{\mathrm{R}_{d}} \mathbf{B B}^{T} \mathbf{A}_{\mathrm{R}_{d}}^{T} \mathbf{g}_{\mathrm{R}_{k}} \\
& =\frac{\sigma_{n}^{2}}{2}\left\|\mathbf{g}_{\mathrm{R}_{k}}^{T} \mathbf{A}_{\mathrm{R}_{d}} \mathbf{B}\right\|_{2}^{2} \\
& =\frac{\sigma_{n}^{2}}{2}\left\|\mathbf{d}_{\mathrm{R}_{k}}\right\|_{2}^{2} \text {, } \\
& E\left[\left|T_{5}\right|^{2}\right]=E\left[\left(\mathbf{g}_{\mathrm{R}_{k}}^{T} \mathbf{n}_{\mathrm{R}_{q, d}}\right)\left(\mathbf{g}_{\mathrm{R}_{k}}^{T} \mathbf{n}_{\mathrm{R}_{q, d}}\right)^{T}\right] \\
& =E\left[\mathbf{g}_{\mathrm{R}_{k}}^{T} \mathbf{n}_{\mathrm{R}_{q, d}} \mathbf{n}_{\mathrm{R}_{q, d}}^{T} \mathbf{g}_{\mathrm{R}_{k}}\right] \\
& =\mathbf{g}_{\mathrm{R}_{k}}^{T} \mathbf{C}_{\mathbf{n}_{\mathrm{R}_{q, d}}} \mathbf{g}_{\mathrm{R}_{k}} \text {, }
\end{aligned}
$$

where due to the real-valued notation of the system $\mathbf{C}_{\mathbf{x}_{\mathrm{R}}}=E\left[\mathbf{x}_{\mathrm{R}} \mathbf{x}_{\mathrm{R}}^{T}\right]=\frac{1}{2} \mathbf{I}_{2 N_{t}}$ and $\mathbf{C}_{\mathbf{n}_{\mathrm{R}_{\mathbf{d}}}}=E\left[\mathbf{n}_{\mathrm{R}_{d}} \mathbf{n}_{\mathrm{R}_{d}}^{T}\right]=\frac{\sigma_{n}^{2}}{2} \mathbf{I}_{2 N_{r}}$. Moreover, $\mathbf{d}_{\mathrm{R}_{k}}=\mathbf{g}_{\mathrm{R}_{k}}^{T} \mathbf{A}_{\mathrm{R}_{d}} \mathbf{B}$.

By replacing (C-11), (C-12), (C-13), (C-14) and (C-15) into (C-5), the $\mathrm{SINR}_{k}$ is expressed by 


$$
\begin{aligned}
\operatorname{SINR}_{k} & =\frac{\frac{1}{2}\left|\mathbf{d}_{\mathrm{R}_{k}} \hat{\mathbf{h}}_{\mathrm{R}_{k}}\right|^{2}}{\frac{1}{2} \sum_{i \neq k}^{K}\left|\mathbf{d}_{\mathrm{R}_{k}} \hat{\mathbf{h}}_{\mathrm{R}_{i}}\right|^{2}+\frac{1}{2} \sum_{i=1}^{K}\left|\mathbf{d}_{\mathrm{R}_{k}} \boldsymbol{\varepsilon}_{\mathrm{R}_{i}}\right|^{2}+\frac{\sigma_{n}^{2}}{2}\left\|\mathbf{d}_{\mathrm{R}_{k}}\right\|_{2}^{2}+\mathbf{g}_{\mathrm{R}_{k}}^{T} \mathbf{C}_{\mathbf{n}_{\mathrm{R}_{q, d}}} \mathbf{g}_{\mathrm{R}_{k}}} \\
& =\frac{\left|\mathbf{d}_{\mathrm{R}_{k}} \hat{\mathbf{h}}_{\mathrm{R}_{k}}\right|^{2}}{\sum_{i \neq k}^{K}\left|\mathbf{d}_{\mathrm{R}_{k}} \hat{\mathbf{h}}_{\mathrm{R}_{i}}\right|^{2}+\sum_{i=1}^{K}\left|\mathbf{d}_{\mathrm{R}_{k}} \boldsymbol{\varepsilon}_{\mathrm{R}_{i}}\right|^{2}+\sigma_{n}^{2}\left\|\mathbf{d}_{\mathrm{R}_{k}}\right\|_{2}^{2}+2 \mathbf{g}_{\mathrm{R}_{k}}^{T} \mathbf{C}_{\mathrm{n}_{\mathrm{R}_{q, d}}} \mathbf{g}_{\mathrm{R}_{k}}}
\end{aligned}
$$

Finally, by replacing (C-16) into (C-3), the ergodic achievable rate per real-valued channel as in (6-5) reads as

$$
I_{\mathrm{R}_{k}}=E\left[\frac{1}{2} \log _{2}\left(1+\frac{\left|\mathbf{d}_{\mathrm{R}_{k}} \hat{\mathbf{h}}_{\mathrm{R}_{k}}\right|^{2}}{\sum_{i \neq k}^{K}\left|\mathbf{d}_{\mathrm{R}_{k}} \hat{\mathbf{h}}_{\mathrm{R}_{i}}\right|^{2}+\sum_{i=1}^{K}\left|\mathbf{d}_{\mathrm{R}_{k}} \varepsilon_{\mathrm{R}_{i}}\right|^{2}+\sigma_{n}^{2}|| \mathbf{d}_{\mathrm{R}_{k}}||_{2}^{2}+2 \mathbf{g}_{\mathrm{R}_{k}}^{T} \mathbf{C}_{\mathrm{R}_{\mathrm{R}_{q, d}}} \mathbf{g}_{\mathrm{R}_{k}}}\right)\right] .
$$

